\title{
On phase-space integrals with Heaviside functions
}

\author{
Daniel Baranowski, ${ }^{a}$ Maximilian Delto, ${ }^{a, b}$ Kirill Melnikov ${ }^{a}$ and Chen-Yu Wang ${ }^{a}$ \\ ${ }^{a}$ Institute for Theoretical Particle Physics, KIT, \\ 76128 Karlsruhe, Germany \\ ${ }^{b}$ Physik Department, Technische Universität München, \\ James-Franck-Straße 1, 85748 Garching, Germany \\ E-mail: daniel.baranowski@kit.edu, maximilian.delto@tum.de, \\ kirill.melnikov@kit.edu, chen-yu.wang@kit.edu
}

ABstract: We discuss peculiarities that arise in the computation of real-emission contributions to observables that contain Heaviside functions. A prominent example of such a case is the zero-jettiness soft function in SCET, whose calculation at next-to-next-to-next-to-leading order in perturbative QCD is an interesting problem. Since the zero-jettiness soft function distinguishes between emissions into different hemispheres, its definition involves $\theta$-functions of light-cone components of emitted soft partons. This prevents a direct use of multi-loop methods, based on reverse unitarity, for computing the zero-jettiness soft function in high orders of perturbation theory. We propose a way to bypass this problem and illustrate its effectiveness by computing various non-trivial contributions to the zero-jettiness soft function at NNLO and N3LO in perturbative QCD.

KEYWORDS: QCD Phenomenology

ArXiv EPrint: 2111.13594 


\section{Contents}

1 Introduction 1

2 Heaviside functions in the zero-jettiness soft function 3

3 Applying integration-by-parts technology to integrals with $\theta$-functions 5

4 IBP identities and the NNLO QCD contribution to the zero-jettiness soft function

4.1 An example of an IBP relation 7

4.2 Reduction to master integrals 9

$\begin{array}{ll}4.3 \text { Computation of master integrals } & 11\end{array}$

$\begin{array}{lll}4.4 & \text { Results for the real emission contribution at NNLO }\end{array}$

5 Testing the method with some N3LO contributions to the zero-jettiness soft function

5.1 The $\omega_{n \bar{n}}^{(3), a}$ contribution $\quad 16$

5.2 The $\omega_{n \bar{n}}^{(3), b}$ contribution $\quad 18$

5.3 The $\omega_{n \bar{n}}^{(3), c}$ contribution 22

5.4 Differential equations and $\omega_{n \bar{n}}^{(3), d}$ contribution 28

5.4.1 Calculation of the boundary conditions 30

5.4.2 Numerical solution of the differential equations 35

6 Conclusion 38

A Master integrals for $n n n$ contribution to the soft function 39

B Matrices for the differential equations in section $\mathbf{5 . 4 . 2} 41$

\section{Introduction}

Precision studies of hadron collisions moved into the focus of the particle physics community after no clear evidence for physics beyond the Standard Model has been found during the first two LHC runs. A pre-requisite for such studies is a solid theoretical framework that allows one to describe hadron collisions using quark and gluon degrees of freedom and, in this way, connect experimental data to the Standard Model Lagrangian without the need for additional modeling. The current theoretical framework is based on the concept of collinear factorization [1] that, for processes with large momentum transfer, relates hadronic cross sections to convolutions of partonic cross sections, computable in perturbation theory, with universal non-perturbative parton distribution functions. Further refinements and practical advancements of such a framework are currently among the central topics in theoretical collider physics. 
Improvements in an existent framework may be provided by the soft-collinear effective theory (SCET) [2-6] that seeks to establish a general pattern of factorization in collider processes including both perturbative and non-perturbative physics. This effective theory defines objects that are sensitive to particular momenta "modes" such as e.g. soft, collinear etc. These objects can be calculated independently of each other and then combined to provide predictions for physical quantities such as cross sections and kinematic distributions. Key to this effort are factorization theorems that both define these objects precisely and also provide information on how physical predictions should be assembled once these objects have been computed.

There are four types of objects that appear in SCET; they are known as hard, beam, jet and soft functions. Theoretical predictions for these functions are of interest since they can be used to re-sum logarithmically-enhanced terms that appear in perturbative expansion in QCD. In addition, they can also be used to set up a slicing method for deriving fixed-order predictions for fully-differential calculations in QCD [7-18].

The slicing method requires an observable which can be used to separate the realemission phase spaces into "singular" and "regular" parts. Although, in principle, any observable can be used to do that, if an observable is chosen in a way that does not violate factorization into collinear and soft modes, a cross section, differential with respect to such an observable, should satisfy a particular factorization theorem. Such a factorization theorem would then contain soft and beam functions whose computation enables both, the construction of subtraction terms for perturbative computations and the re-summation of large logarithms that arise in theoretical predictions once the slicing variable becomes small.

In this paper we will deal with the so called zero-jettiness variable $[19,20]$ that can be used as a slicing variable for processes where a color-less final state is produced in hadron collisions. Calculations of the corresponding soft and beam functions have been performed during the past decade. The zero-jettiness soft function has been computed through NNLO QCD in refs. [21, 22] (see also refs. [23, 24]). The zero-jettiness beam function has been calculated through NNLO QCD in refs. [25-27]. Studies of zero-jettiness beam functions at N3LO QCD were initiated in refs. [24, 28-30] and were recently completed in refs. [31, 32]. ${ }^{1}$

In this paper, we focus on certain technical aspects that arise in the computation of the zero-jettiness soft function in higher orders of perturbative QCD. Due to algebraic complexity of computations in high orders of perturbation theory, specialized tools and methods are usually employed. Chief among them is the integration-by-parts (IBP) method for loop integrals introduced in ref. [43] and adapted to real-emission integrals in ref. [44]. However, application of the IBP method to the computation of the zero-jettiness soft function is not straightforward, as the zero-jettiness observable contains Heaviside functions that depend on the light-cone components of four-momenta of the emitted partons. ${ }^{2}$ This fact, as well as the need to compute large number of complicated integrals, makes the

\footnotetext{
${ }^{1}$ Jettiness soft functions for more complicated final states were studied in refs. [33-36]. Beam functions for $q_{T}$ factorization were calculated in refs. [37-40]. Automated approaches to computation of soft, beam and jet functions are discussed in refs. [41, 42].

${ }^{2}$ We note that Heaviside functions can also appear in the construction of NNLO subtraction terms that need to be integrated over unresolved phase spaces of final state particles, see e.g. refs. [45-47].
} 
calculation of N3LO soft function non-trivial. Our goal in this paper is to discuss possible ways to overcome these technical difficulties paving the way for the computation of the triple-real and real-virtual contributions to the N3LO soft function. As a proof of concept, we compute a number of non-trivial contributions to the N3LO soft function, that describe emission of three gluons into the same hemisphere.

The remainder of this paper is organized as follows. In section 2 we introduce the zero-jettiness soft function. In section 3 we explain how integration by parts can be used to simplify computation of integrals with Heaviside functions. In section 4, we apply this method to compute the soft function at next-to-next-to-leading order in QCD. We then discuss various aspects of the N3LO calculation in section 5 . We conclude in section 6 . Some useful formulas are collected in appendices A and B.

\section{Heaviside functions in the zero-jettiness soft function}

In this section we will briefly discuss the zero-jettiness soft function to explain how Heaviside functions appear in phase-space integrals. Consider a process where two partons with (normalized) momenta $n$ and $\bar{n}$ collide and produce a color-neutral final state together with $m$ QCD partons with momenta $k_{1,2, ., m}$. The jettiness variable $\tau$ is defined as follows

$$
\tau=\sum_{j=1}^{m} \min _{q \in\{n, \bar{n}\}}\left[\frac{2 q k_{j}}{n \bar{n}}\right] .
$$

To compute the minima in eq. (2.1), we use the Sudakov parameterization of the parton's momenta and write

$$
k_{i}=\frac{\alpha_{i}}{2} n+\frac{\beta_{i}}{2} \bar{n}+k_{\perp, i}, \quad i=1, \ldots, m,
$$

where $k_{\perp, i} n=k_{\perp, i} \bar{n}=0, n^{2}=\bar{n}^{2}=0$ and $n \bar{n}=2$. It follows that

$$
\tau=\sum_{i=1}^{m} \min \left\{\alpha_{i}, \beta_{i}\right\}
$$

To enable the choice between $\alpha_{i}$ and $\beta_{i}$ in the above equation, we can partition the phase space by writing

$$
1=\theta\left(\alpha_{i}-\beta_{i}\right)+\theta\left(\beta_{i}-\alpha_{i}\right),
$$

for each of the $m$ partons. ${ }^{3}$ It is clear that the first term will contribute $\beta_{i}$ and the second one $\alpha_{i}$ to the jettiness variable $\tau$.

Inserting the partition eq. (2.4) into integrals over momenta of final-state gluons we find phase-space integrals with Heaviside functions. In general, for $m$-emitted gluons we can obtain $2^{m}$ different terms however, using the symmetry between $m$ gluons and the fact that the result is invariant under the simultaneous replacement of all $\alpha_{i}$ 's with $\beta_{i}$ 's and vice versa, the number of different terms is dramatically reduced. For example, for $m \leq 3$ which covers NLO, NNLO and N3LO cases, only two independent contributions to the

\footnotetext{
${ }^{3}$ In what follows, we will focus on gluon emissions.
} 
soft-function need to be considered. They are 1) all $m$ gluons are emitted into the same hemisphere and 2) all but one gluons are emitted into the same hemisphere.

These different cases can be described as integrals over the following phase spaces

$$
\mathrm{d} \Phi_{f_{1}, \ldots, f_{m}}^{h_{1}, \ldots, h_{m}}=\left(N_{\epsilon}\right)^{-m}\left(\prod_{j=1}^{m}\left[\mathrm{~d} k_{j}\right] f_{j}\left(\Delta_{j, h_{j}}\right)\right) \delta\left(1-\sum_{j=1}^{m} \kappa_{j, h_{j}}\right),
$$

where

$$
N_{\epsilon}=\frac{(4 \pi)^{-\epsilon}}{16 \pi^{2} \Gamma(1-\epsilon)}
$$

and

$$
\Delta_{j, h_{j}}=\left\{\begin{array}{ll}
\alpha_{j}-\beta_{j}, & h_{j}=n, \\
\beta_{j}-\alpha_{j}, & h_{j}=\bar{n},
\end{array} \quad \text { and } \quad \kappa_{j, h_{j}}= \begin{cases}\beta_{j}, & h_{j}=n \\
\alpha_{j}, & h_{j}=\bar{n}\end{cases}\right.
$$

Thanks to the definition of the jettiness variable, we associate functions $f_{j}$ in eq. (2.5) with Heaviside functions but, as we will see later, we will also need to consider cases where one or several of these functions $f$ are $\delta$-functions.

To explicitly see how these phase spaces are used, we consider an example of the real-emission contribution to the soft function. Then, for NNLO and N3LO computations we require the following integrals

$$
S_{n n}=\int \mathrm{d} \Phi_{\theta \theta}^{n n} \operatorname{Eik}_{2 g}\left(\left\{k_{i}\right\}, n, \bar{n}\right), \quad S_{n \bar{n}}=\int \mathrm{d} \Phi_{\theta \theta}^{n \bar{n}} \operatorname{Eik}_{2 g}\left(\left\{k_{i}\right\}, n, \bar{n}\right),
$$

and

$$
S_{n n n}=\int \mathrm{d} \Phi_{\theta \theta \theta}^{n n n} \operatorname{Eik}_{3 g}\left(\left\{k_{i}\right\}, n, \bar{n}\right), \quad S_{n n \bar{n}}=\int \mathrm{d} \Phi_{\theta \theta \theta}^{n n \bar{n}} \operatorname{Eik}_{3 g}\left(\left\{k_{i}\right\}, n, \bar{n}\right),
$$

where $\operatorname{Eik}_{3 g}\left(\left\{k_{i}\right\}\right.$ and $\operatorname{Eik}_{2 g}\left(\left\{k_{i}\right\}\right.$ are properly rescaled three-gluon and two-gluon eikonal functions. We will specify these functions later. For now, suffice it to say that they depend on the scalar products of gluon four-momenta $k_{i} k_{j}$ and on scalar products of gluon four-momenta with external vectors $n$ and $\bar{n}$.

A standard way to simplify computation of complicated phase-space integrals is to use reverse unitarity [44] to map such integrals onto cut loop integrals for which IBP identities can be derived in a straightforward manner. This is achieved by using the formula

$$
\delta(P(\vec{x})) \rightarrow \frac{i}{2 \pi}\left[\frac{1}{P(\vec{x})+i 0}-\frac{1}{P(\vec{x})-i 0}\right],
$$

where $P(\vec{x})$ is a polynomial in variables $\vec{x}$ that can be e.g certain components of gluons' momenta. Once all $\delta$-function constraints in phase-space integrals are removed using eq. (2.10) and, provided, that there are no other non-polynomial constraints in the integrands, one can make use of the powerful integration-by-parts technology [43] to reduce computation of a large number of phase-space integrals to a few master integrals.

Unfortunately, if integrands contain Heaviside functions, this approach fails since the last condition mentioned in the previous paragraph is not fulfilled. A possible solution to 
this problem was pointed out by one of us in ref. [24], where it was suggested to rewrite all $\theta$-functions that appear in relevant integrals as follows ${ }^{4}$

$$
\theta\left(b_{i}-a_{i}\right)=\int_{0}^{1} \mathrm{~d} z_{i} \delta\left(z_{i} b_{i}-a_{i}\right) b_{i}, \quad a_{i}, b_{i}>0 .
$$

While this representation yields an integrand whose dependence on auxiliary variables $z_{i}$ can be computed using reverse unitarity, it also introduces one additional parametric integral per $\theta$-function, which can become quite cumbersome. For this reason, in this paper we would like to investigate how to derive and use IBP relations for phase-space integrals with $\theta$-functions directly, i.e. without the need to introduce additional variables.

\section{Applying integration-by-parts technology to integrals with $\theta$-functions}

The goal of reverse unitarity [44] is to turn phase-space integrals into loop integrals. We explained the main idea of the method in the previous section; we will now make this discussion more specific considering the zero-jettiness soft function.

To remove all $\delta$-function constraints from the integration measure, we start with a phase-space element of a gluon $i$ with momentum $k_{i}^{\mu}=\left(E_{i}, \vec{k}_{i}\right)$ and write it as

$$
\left[\mathrm{d} k_{i}\right]=\frac{\mathrm{d}^{d-1} \vec{k}_{i}}{(2 \pi)^{d-1} 2 E_{i}}=\frac{\mathrm{d}^{d} k_{i}}{(2 \pi)^{d}} 2 \pi \delta_{+}\left(k_{i}^{2}\right)
$$

We then re-write the $\delta$-function as in eq. (2.10)

$$
\delta\left(k_{i}^{2}\right)=\frac{i}{2 \pi}\left(\frac{1}{k_{i}^{2}+i 0}-\frac{1}{k_{i}^{2}-i 0}\right)=\frac{1}{\left[k_{i}^{2}\right]_{c}} .
$$

In addition, to deal with soft functions shown in eqs. (2.8), (2.9) we need to re-write all $\delta$-functions that define the re-scaled jettiness as cut propagators. For example, in case of the $n n \bar{n}$ kinematic configuration, we write

$$
\delta\left(1-k_{12} n-k_{3} \bar{n}\right)=\frac{1}{\left[1-k_{12} n-k_{3} \bar{n}\right]_{c}},
$$

where $k_{12}=k_{1}+k_{2}$.

Hence, if we ignore $\theta$-functions in the integrands of $S_{n n}, S_{n \bar{n}}, S_{n n n}, S_{n n \bar{n}}$, we immediately recognize that we need to compute a collection of "loop" integrals with conventional and unconventional cut "propagators". To do that, we can apply integration-by-parts identities [43] to reduce the number of independent integrals that need to be calculated. Furthermore, there are powerful public programs such as Fire [48, 49], Kira [50, 51], LiteRed [52, 53], and Reduze $[54,55]$ that can perform reductions to master integrals in a highly automated and efficient fashion.

However, if relevant integrands contain a collection of $\theta$-functions, as is indeed the case for the soft function, this procedure can not be applied. An obvious problem is that

\footnotetext{
${ }^{4}$ See also section 4.2 .2 in ref. [38].
} 
$\theta$-functions cannot be turned into "propagators" since the mapping similar to the one shown in eq. (3.3) does not exist. However, we would like to understand what happens if we ignore this problem and attempt to derive IBP identities for integrands with $\theta$-functions.

To study this question, we consider the following integral

$$
I[\theta(f), g]=\int \mathrm{d}^{d} k \theta(f(k)) g(k),
$$

where $f(k)$ is a polynomial in momentum $k$ and $g(k)$ is a function that allows a standard derivation of integration-by-parts identities. ${ }^{5}$ To derive integration-by-parts identities for the integral $I[\theta(f), g]$, we write the standard equation

$$
0=\int \mathrm{d}^{d} k \frac{\partial}{\partial k^{\mu}}\left[v^{\mu} \theta(f(k)) g(k)\right],
$$

that is valid for dimensionally-regularized integrals. Vector $v^{\mu}$ in eq. (3.5) is an arbitrary vector that we do not need to specify further. Calculating the derivative, we obtain

$$
\frac{\partial}{\partial k^{\mu}}\left\{v^{\mu} \theta(f(k)) g(k)\right\}=\theta(f(k)) \frac{\partial}{\partial k^{\mu}}\left\{v^{\mu} g(k)\right\}+\left\{g(k) \delta(f(k)) v^{\mu}\right\} \frac{\partial f(k)}{\partial k_{\mu}} .
$$

It follows that

$$
0=I\left[\theta(f), \partial_{\mu}\left(v^{\mu} g\right)\right]+I\left[\delta(f), g v^{\mu}\left(\partial_{\mu} f\right)\right] .
$$

The first term on the right-hand side belongs to the same class of integrals as the original one $I[\theta(f), g]$ because it involves the same $\theta$-function; we call this term the homogeneous part of the IBP relation. Since, by assumption, integrals of $g(k)$ can be studied using standard integration-by-parts technology, it follows that the homogeneous terms produce a closed set of linear equations when studied on their own.

The second term in eq. (3.7) involves $\delta(f)$; we call this term the inhomogeneous part of the IBP relation. Since we can use the generalized unitarity trick to write $\delta(f) \rightarrow 1 / f$ and since $f$ is a polynomial in $k, I\left[\delta(f), g v^{\mu} \partial_{\mu} f\right]$ defines a class of integrals that can be studied on their own independent of integrals with $\theta$-functions. In fact, obtaining integration-by-parts identities for this class of integrals can be done with standard methods. The only subtlety that we have to deal with when working with inhomogeneous terms is that the function $g(k) / f(k)$ may contain linearly-dependent "propagators" that will have to be re-mapped onto properly-defined integral families. Although this, by itself, is not a crucial issue, it does not allow us to derive IBP relations for integrals with arbitrary powers of propagators and forces us to produce IBP relations for each of the seed integrals individually.

We thus conclude that it is possible to establish useful integration-by-parts identities for integrals with multiple $\theta$-functions by iteratively using eq. (3.7). It follows from that equation that the derivative of an integrand produces inhomogeneous terms, where a $\theta$-function is replaced by a $\delta$-function.

Therefore, by using eq. (3.7) repeatedly, we obtain a hierarchical sequence of IBP relations containing integrals with a decreasing number of $\theta$-functions and an increasing number of $\delta$-functions. The IBP relations can be used to express all relevant integrals

\footnotetext{
${ }^{5}$ In case of the soft functions, $f(k)$ reads $f(k)= \pm(k \bar{n}-k n)$.
} 
through a set of master integrals. When choosing master integrals, we try to select those that contain fewer $\theta$-functions since they are easier to compute. We will illustrate the construction of IBP relations and their usage in the next section where we will calculate the real-emission contribution to the zero-jettiness soft function at NNLO.

\section{IBP identities and the NNLO QCD contribution to the zero-jettiness soft function}

In this section, we show how to use reverse unitarity and modified IBP relations to compute the maximally non-abelian contribution to zero-jettiness soft function at NNLO. We define it as

$$
S_{\mathrm{NA}}^{2 g}=\frac{1}{\tau^{1+4 \epsilon}}\left[\int \mathrm{d} \Phi_{\theta \theta}^{n n} \omega_{n \bar{n}}^{(2)}\left(k_{1}, k_{2}\right)+\int \mathrm{d} \Phi_{\theta \theta}^{n \bar{n}} \omega_{n \bar{n}}^{(2)}\left(k_{1}, k_{2}\right)\right],
$$

where $[56]$

$$
\omega_{n \bar{n}}^{(2)}\left(k_{1}, k_{2}\right)=S_{n \bar{n}}\left(k_{1}, k_{2}\right)+S_{\bar{n} n}\left(k_{1}, k_{2}\right)-S_{n n}\left(k_{1}, k_{2}\right)-S_{\bar{n} \bar{n}}\left(k_{1}, k_{2}\right)
$$

with

$$
\begin{aligned}
\mathcal{S}_{p_{i} p_{j}}\left(k_{1}, k_{2}\right)= & \frac{(1-\epsilon)}{\left(k_{1} \cdot k_{2}\right)^{2}} \frac{\left[\left(p_{i} \cdot k_{1}\right)\left(p_{j} \cdot k_{2}\right)+i \leftrightarrow j\right]}{\left(p_{i} \cdot k_{12}\right)\left(p_{j} \cdot k_{12}\right)} \\
& -\frac{\left(p_{i} \cdot p_{j}\right)^{2}}{2\left(p_{i} \cdot k_{1}\right)\left(p_{j} \cdot k_{2}\right)\left(p_{i} \cdot k_{2}\right)\left(p_{j} \cdot k_{1}\right)}\left[2-\frac{\left[\left(p_{i} \cdot k_{1}\right)\left(p_{j} \cdot k_{2}\right)+i \leftrightarrow j\right]}{\left(p_{i} \cdot k_{12}\right)\left(p_{j} \cdot k_{12}\right)}\right] \\
+\frac{\left(p_{i} \cdot p_{j}\right)}{2\left(k_{1} \cdot k_{2}\right)}\left[\frac{2}{\left(p_{i} \cdot k_{1}\right)\left(p_{j} \cdot k_{2}\right)}+\frac{2}{\left(p_{j} \cdot k_{1}\right)\left(p_{i} \cdot k_{2}\right)}-\frac{1}{\left(p_{i} \cdot k_{12}\right)\left(p_{j} \cdot k_{12}\right)}\right. & \\
& \left.\times\left(4+\frac{\left[\left(p_{i} \cdot k_{1}\right)\left(p_{j} \cdot k_{2}\right)+i \leftrightarrow j\right]^{2}}{\left(p_{i} \cdot k_{1}\right)\left(p_{j} \cdot k_{2}\right)\left(p_{i} \cdot k_{2}\right)\left(p_{j} \cdot k_{1}\right)}\right)\right] .
\end{aligned}
$$

In the next section we explicitly construct a few examples of IBP equations with $\theta$-functions that are relevant for this case. We discuss the computation of the two terms in eq. (4.1) after that.

\subsection{An example of an IBP relation}

In this section, we explain how to employ modified integration-by-parts identities discussed in section 3. As the first step, we map all integrals that appear in eq. (4.1) onto integral families. These families are defined by sets of linearly-independent propagators ${ }^{6}$ and additionally contain two $\theta$-functions from phase-space measures in eq. (4.1). Cut propagators are constructed from $\delta$-functions $\delta\left(k_{i}^{2}\right)$ that enforce the on-shell conditions for the emitted gluons, and also from jettiness-dependent $\delta$-functions that appear in the corresponding phase spaces

$$
\begin{aligned}
& \mathrm{d} \Phi_{\theta \theta}^{n n} \sim \delta\left(1-\beta_{1}-\beta_{2}\right)=\delta\left(1-k_{1} n-k_{2} n\right), \\
& \mathrm{d} \Phi_{\theta \theta}^{n \bar{n}} \sim \delta\left(1-\beta_{1}-\alpha_{2}\right)=\delta\left(1-k_{1} n-k_{2} \bar{n}\right) .
\end{aligned}
$$

\footnotetext{
${ }^{6}$ When referring to propagators, we imply both cut and ordinary ones. In case of the real-emission contribution to NNLO soft function, we have three cut propagators and four ordinary ones.
} 
After partial fractioning, we find that we need several independent integral families in this case. For example, one integral family that is required to describe the $n n$-configuration reads

$$
\mathcal{T}_{a_{1} \ldots a_{7}}^{\mathrm{ex}}=\int \frac{\mathrm{d}^{d} k_{1} \mathrm{~d}^{d} k_{2} \theta\left(k_{1} \bar{n}-k_{1} n\right) \theta\left(k_{2} \bar{n}-k_{2} n\right)}{\left[\left(k_{1}^{2}\right)^{a_{1}}\left(k_{2}^{2}\right)^{a_{2}}\left(1-k_{12} n\right)^{a_{3}}\right]_{c}\left(k_{1} k_{2}\right)^{a_{4}}\left(k_{2} n\right)^{a_{5}}\left(k_{1} \bar{n}\right)^{a_{6}}\left(k_{12} \bar{n}\right)^{a_{7}}},
$$

where the subscript $c$ denotes cut propagators.

In order to construct an explicit example of the modified IBP relations discussed in section 3 , we consider the integral

$$
\mathcal{I}_{\text {ex }}=\int \frac{\mathrm{d} \Phi_{\theta \theta}^{n n}}{\left(k_{2} n\right)\left(k_{1} \bar{n}\right)\left(k_{12} \bar{n}\right)}=\mathcal{T}_{1,1,1,0,1,1,1}^{\mathrm{ex}} .
$$

Starting with this "seed integral" and following the discussion around eq. (3.5), we derive eight different equations by computing derivatives w.r.t. $k_{1}$ and $k_{2}$ and by using vectors $v \in\left\{k_{1}, k_{2}, n, \bar{n}\right\}$. For example, differentiating w.r.t. $k_{1}$ and choosing $v=k_{1}$, we find

$$
\begin{aligned}
0= & \int \mathrm{d}^{d} k_{1} \mathrm{~d}^{d} k_{2} \frac{\partial}{\partial k_{1}^{\mu}} \frac{k_{1}^{\mu} \theta\left(k_{1} \bar{n}-k_{1} n\right) \theta\left(k_{2} \bar{n}-k_{2} n\right)}{\left(k_{1}^{2}\right)\left(k_{2}^{2}\right)\left(1-k_{12} n\right)\left(k_{2} n\right)\left(k_{1} \bar{n}\right)\left(k_{12} \bar{n}\right)} \\
= & (d-4) \mathcal{T}_{1,1,1,0,1,1,1}^{\mathrm{ex}}-\mathcal{T}_{1,1,1,0,1,0,2}^{\mathrm{ex}}-\mathcal{T}_{1,1,2,0,0,1,1}^{\mathrm{ex}}+\mathcal{T}_{1,1,2,0,1,1,1}^{\mathrm{ex}} \\
& +\int \mathrm{d}^{d} k_{1} \mathrm{~d}^{d} k_{2} \frac{k_{1}^{\mu}(\bar{n}-n)_{\mu} \delta\left(k_{1} \bar{n}-k_{1} n\right) \theta\left(k_{2} \bar{n}-k_{2} n\right)}{\left(k_{1}^{2}\right)\left(k_{2}^{2}\right)\left(1-k_{12} n\right)\left(k_{2} n\right)\left(k_{1} \bar{n}\right)\left(k_{12} \bar{n}\right)}
\end{aligned}
$$

In writing this equation, we have used the fact that homogeneous terms, which arise when the derivative does not act on the $\theta$-function, belong to the same family of integrals as the seed integral.

As we already mentioned, inhomogeneous terms arise when derivatives act on the $\theta$-functions. In our example the last term in eq. (4.8) is inhomogeneous. This term vanishes because $k_{1}^{\mu}(\bar{n}-n)_{\mu} \delta\left(k_{1} \bar{n}-k_{1} n\right)=0$. This is a general feature; indeed, we find that integration-by-parts identities that involve a differential operator $k_{i}^{\mu} \partial / \partial k_{i, \mu}$ do not produce inhomogeneous terms. To prove that assertion, consider

$$
\frac{\partial}{\partial k_{i}^{\mu}}\left[k_{i}^{\mu} \theta\left(k_{i}^{\mu} q_{\mu}\right) g(k)\right]=\theta\left(k_{i}^{\mu} q_{\mu}\right) \frac{\partial}{\partial k_{i}^{\mu}}\left[k_{i}^{\mu} g(k)\right]+[g(k)] k_{i}^{\mu} q_{\mu} \delta\left(k_{i}^{\mu} q_{\mu}\right) .
$$

The second term on the right hand side of eq. (4.9) vanishes which implies that differential operators of the form $k_{i}^{\mu} \partial / \partial k_{i}^{\mu}$ do not produce inhomogeneous contributions.

As a second example, we consider the derivative w.r.t. $k_{1}$ and use $v=n$. We find

$$
\begin{aligned}
0= & \int \mathrm{d}^{d} k_{1} \mathrm{~d}^{d} k_{2} n^{\mu} \frac{\partial}{\partial k_{1}^{\mu}} \frac{\theta\left(k_{1} \bar{n}-k_{1} n\right) \theta\left(k_{2} \bar{n}-k_{2} n\right)}{\left(k_{1}^{2}\right)\left(k_{2}^{2}\right)\left(1-k_{12} n\right)\left(k_{1} \bar{n}\right)\left(k_{2} n\right)\left(k_{12} \bar{n}\right)} \\
= & 2 \mathcal{T}_{1,1,1,0,1,1,2}^{\mathrm{ex}}-2 \mathcal{T}_{1,1,1,0,1,2,1}^{\mathrm{ex}}+2 \mathcal{T}_{2,1,1,0,0,1,1}^{\mathrm{ex}}-2 \mathcal{T}_{2,1,1,0,1,1,1}^{\mathrm{ex}} \\
& +(n \bar{n}) \int \mathrm{d}^{d} k_{1} \mathrm{~d}^{d} k_{2} \frac{\delta\left(k_{1} \bar{n}-k_{1} n\right) \theta\left(k_{2} \bar{n}-k_{2} n\right)}{\left(k_{1}^{2}\right)\left(k_{2}^{2}\right)\left(1-k_{12} n\right)\left(k_{1} \bar{n}\right)\left(k_{2} n\right)\left(k_{12} \bar{n}\right)} .
\end{aligned}
$$

Similar to the previous case, we have expressed homogeneous terms through integrals of the family $\mathcal{T}^{\mathrm{ex}}$. However, in this second example, the inhomogeneous term does not vanish and requires further treatment in the IBP setup. 
The reason for that is the fact that the propagators in that term are linearly dependent due to the new $\delta$-function in the integrand $\delta\left(k_{1} \bar{n}-k_{1} n\right)$ that becomes a rational function if reverse unitarity is used. To see this explicitly, we make the replacement $\delta\left(k_{1} \bar{n}-k_{1} n\right) \rightarrow$ $1 /\left[k_{1} \bar{n}-k_{1} n\right]_{c}$, multiply the result with the partial fractioning identity

$$
1=\frac{\left(k_{1} \bar{n}\right)-\left[k_{1} \bar{n}-k_{1} n\right]_{c}}{\left(k_{1} n\right)} \times\left\{\left[1-k_{12} n\right]_{c}+\left(k_{1} n\right)+\left(k_{2} n\right)\right\}
$$

and obtain

$$
\begin{aligned}
& \int \frac{\mathrm{d}^{d} k_{1} \mathrm{~d}^{d} k_{2} \theta\left(k_{2} \bar{n}-k_{2} n\right)}{\left[\left(k_{1}^{2}\right)\left(k_{2}^{2}\right)\left(1-k_{12} n\right)\left(k_{1} \bar{n}-k_{1} n\right)\right]_{c}\left(k_{1} \bar{n}\right)\left(k_{2} n\right)\left(k_{12} \bar{n}\right)} \\
& \quad=\int \frac{\mathrm{d}^{d} k_{1} \mathrm{~d}^{d} k_{2} \theta\left(k_{2} \bar{n}-k_{2} n\right)}{\left[\left(k_{1}^{2}\right)\left(k_{2}^{2}\right)\left(1-k_{12} n\right)\left(k_{1} \bar{n}-k_{1} n\right)\right]_{c}\left(k_{12} \bar{n}\right)}\left[\frac{1}{\left(k_{1} n\right)}+\frac{1}{\left(k_{2} n\right)}\right] .
\end{aligned}
$$

We note that terms that do not contain the complete set of cut propagators were set to zero in the above equation. Furthermore, we note that it is the partial fractioning step that prevents us from writing IBP relations for arbitrary powers of propagators, as it is usually done when traditional IBP relations are derived for integrals without $\theta$-functions. Because of this, we need to generate equations by selecting seed integrals, deriving IBP relations for them and explicitly mapping all inhomogeneous terms to simpler integral families. We further elaborate on this point in the next section.

In summary, we found that modified IBP relations in the considered case relate integrals with two $\theta$-functions to integrals with one $\theta$-function. Written as phase-space integrals, the modified IBP relations read

$$
\begin{aligned}
& 0=\sum_{i} a_{i}^{\text {hom }}(\epsilon) \int \mathrm{d} \Phi_{\theta \theta}^{h_{1} h_{2}} g_{i}\left(n, \bar{n}, k_{1}, k_{2}\right)+\sum_{i} a_{i}^{\text {inhom }}(\epsilon) \int \mathrm{d} \Phi_{\delta \theta}^{h_{1} h_{2}} g_{i}\left(n, \bar{n}, k_{1}, k_{2}\right), \\
& 0=\sum_{i} b_{i}^{\text {hom }}(\epsilon) \int \mathrm{d} \Phi_{\theta \theta}^{h_{1} h_{2}} g_{i}\left(n, \bar{n}, k_{1}, k_{2}\right)+\sum_{i} b_{i}^{\text {inhom }}(\epsilon) \int \mathrm{d} \Phi_{\theta \delta}^{h_{1} h_{2}} g_{i}\left(n, \bar{n}, k_{1}, k_{2}\right),
\end{aligned}
$$

where the two equations arise from derivatives w.r.t. $k_{1}$ and $k_{2}$, respectively.

We can apply the same logic to integrals with a $\theta$-function and a $\delta$-function. Inhomogeneous terms in this case will contain two $\delta$-functions and no $\theta$-function. IBP relations for such integrals can be derived using conventional methods since $\delta$-function constraints can be immediately mapped onto rational functions of parton momenta using reverse unitarity.

\subsection{Reduction to master integrals}

In the previous section, we presented an explicit example of a modified IBP relation that we derived starting with a seed integral with two $\theta$-functions. We note that modified IBP relations naturally form a hierarchical structure since the smaller the number of $\theta$-functions that a particular integral contains, the easier it is to compute it. Hence, in the course of the reduction, we try to express integrals with larger number of $\theta$-functions through integrals with some of the $\theta$-functions replaced by the $\delta$-functions. Integrals that belong to the same "hierarchical level" are organized into distinct integral families. 
In practice, we implement derivation of modified IBP relations in Mathematica and solve them using the "user-defined system" functionality of Kira. This requires us to define integral families with different numbers of $\theta$ - and $\delta$-functions and to derive relations among integrals that belong to different families. We note that for conventional integrals all these steps are done automatically by publicly-available reduction programs, but we have to take care of them ourselves in the present case.

As we already mentioned, it is straightforward to derive homogeneous relations for integrals in each family for arbitrary powers of propagators. For example, for the integral family defined in eq. (4.6), the derivative with respect to $k_{2}$, contracted with $v=\bar{n}$ yields ${ }^{7}$

$$
\begin{aligned}
0 & =\int \mathrm{d}^{d} k_{1} \mathrm{~d}^{d} k_{2} \bar{n}^{\mu} \frac{\partial}{\partial k_{2}^{\mu}} \frac{\theta\left(k_{1} \bar{n}-k_{1} n\right) \theta\left(k_{2} \bar{n}-k_{2} n\right)}{[\ldots]_{c}\left(k_{1} k_{2}\right)^{a_{4}}\left(k_{2} n\right)^{a_{5}}\left(k_{1} \bar{n}\right)^{a_{6}}\left(k_{12} \bar{n}\right)^{a_{7}}} \\
& =\left[2 a_{2} \hat{2}^{+}\left(\hat{6}^{-}-\hat{7}^{-}\right)+2 a_{3} \hat{3}^{+}-a_{4} \hat{4}^{+} \hat{6}^{-}-2 a_{5} \hat{5}^{+}\right] \mathcal{T}_{a_{1}, a_{2}, a_{3}, a_{4}, a_{5}, a_{6}, a_{7}}^{\mathrm{ex}}+\ldots
\end{aligned}
$$

In writing this equation, we have defined operators $\hat{i}^{+(-)}$that raise (lower) the index $a_{i}$ of the integral $\mathcal{T}_{a_{1}, a_{2}, a_{3}, a_{4}, a_{5}, a_{6}, a_{7}}^{\mathrm{ex}}$ by one.

Inhomogeneous terms, such as the one that appeared in eq. (4.10) and discussed after that equation, are generated on an integral-by-integral basis; they require partial fraction decomposition and "on-the-fly" matching to topologies with fewer $\theta$-functions. Although this step is straightforward, it needs to be implemented in a separate Mathematica code whose output is then fed to Kira.

Using this setup, we express the maximally non-abelian contribution to the zero-jettiness soft function defined in eq. (4.1) in terms of eleven master integrals

$$
\begin{aligned}
S_{\mathrm{NA}}^{2 g}=\tau^{-1-4 \epsilon}\left\{\left[\frac{\left(192 \epsilon^{5}+48 \epsilon^{4}-736 \epsilon^{3}+1336 \epsilon^{2}-376 \epsilon+33\right)}{3 \epsilon^{3}(2 \epsilon-3)(2 \epsilon-1)} \mathcal{I}_{1}^{n n}\right.\right. \\
\left.\quad-\frac{8(4 \epsilon-1)\left(12 \epsilon^{4}-25 \epsilon^{2}+41 \epsilon-3\right)}{3 \epsilon^{2}(2 \epsilon-3)(2 \epsilon-1)} \mathcal{I}_{2}^{n n}+\frac{3}{\epsilon} \mathcal{I}_{3}^{n n}+\frac{2}{\epsilon} \mathcal{I}_{4}^{n n}\right] \\
+\left[\frac{128 \epsilon^{7}+864 \epsilon^{6}-848 \epsilon^{5}-1680 \epsilon^{4}+152 \epsilon^{3}+770 \epsilon^{2}-163 \epsilon+3}{\epsilon^{3}(\epsilon+1)(2 \epsilon-1)(2 \epsilon+1)(2 \epsilon+3)} \mathcal{I}_{1}^{n \bar{n}}\right. \\
\quad-\frac{8\left(64 \epsilon^{7}+120 \epsilon^{6}-164 \epsilon^{5}-246 \epsilon^{4}+69 \epsilon^{3}+126 \epsilon^{2}-46 \epsilon+3\right)}{\epsilon^{2}(\epsilon+1)(2 \epsilon-1)(2 \epsilon+1)(2 \epsilon+3)} \mathcal{I}_{2}^{n \bar{n}} \\
\quad+\frac{\left(16 \epsilon^{5}+16 \epsilon^{3}+36 \epsilon^{2}+11 \epsilon-9\right)}{\epsilon(\epsilon+1)(2 \epsilon+1)(2 \epsilon+3)} \mathcal{I}_{3}^{n \bar{n}}+\frac{2}{\epsilon} \mathcal{I}_{4}^{n \bar{n}} \\
\left.\left.\quad-\frac{8(4 \epsilon-1)\left(2 \epsilon^{3}+3 \epsilon^{2}+3 \epsilon-3\right)}{\epsilon(2 \epsilon+1)(2 \epsilon+3)} \mathcal{I}_{5}^{n \bar{n}}+2 \mathcal{I}_{6}^{n \bar{n}}+4 \mathcal{I}_{7}^{n \bar{n}}\right]\right\} .
\end{aligned}
$$

The master integrals that appear in eq. (4.16) read

$$
\begin{array}{ll}
\mathcal{I}_{1}^{n n}=\int \mathrm{d} \Phi_{\delta \delta}^{n n}, & \mathcal{I}_{2}^{n n}=\int \frac{\mathrm{d} \Phi_{\delta \theta}^{n n}}{\left(k_{12} \bar{n}\right)}, \\
\mathcal{I}_{3}^{n n}=\int \frac{\mathrm{d} \Phi_{\delta \theta}^{n n}}{\left(k_{1} k_{2}\right)\left(k_{2} \bar{n}\right)}, & \mathcal{I}_{4}^{n n}=\int \frac{\mathrm{d} \Phi_{\delta \theta}^{n n}}{\left(k_{1} k_{2}\right)\left(k_{2} n\right)\left(k_{12} \bar{n}\right)},
\end{array}
$$

\footnotetext{
${ }^{7}$ We neglect inhomogeneous terms, represented by the ellipses.
} 
and

$$
\begin{array}{rlrl}
\mathcal{I}_{1}^{n \bar{n}} & =\int \mathrm{d} \Phi_{\delta \delta}^{n \bar{n}}, & \mathcal{I}_{2}^{n \bar{n}} & =\int \frac{\mathrm{d} \Phi_{\delta \theta}^{n \bar{n}}}{\left(k_{12} n\right)}, \\
\mathcal{I}_{3}^{n \bar{n}} & =\int \frac{\mathrm{d} \Phi_{\delta \theta}^{n \bar{n}}}{\left(k_{1} k_{2}\right)\left(k_{2} n\right)}, & \mathcal{I}_{4}^{n \bar{n}} & =\int \frac{\mathrm{d} \Phi_{\delta \theta}^{n \bar{n}}}{\left(k_{1} k_{2}\right)\left(k_{2} \bar{n}\right)\left(k_{12} n\right)}, \\
\mathcal{I}_{5}^{n \bar{n}} & =\int \frac{\mathrm{d} \Phi_{\theta \theta}^{n \bar{n}}}{\left(k_{12} n\right)\left(k_{12} \bar{n}\right)}, & \mathcal{I}_{6}^{n \bar{n}} & =\int \frac{\mathrm{d} \Phi_{\theta \theta}^{n \bar{n}}}{\left(k_{1} k_{2}\right)\left(k_{1} \bar{n}\right)\left(k_{2} n\right)}, \\
\mathcal{I}_{7}^{n \bar{n}} & =\int \frac{\mathrm{d} \Phi_{\theta \theta}^{n \bar{n}}}{\left(k_{1} k_{2}\right)\left(k_{2} n\right)\left(k_{12} \bar{n}\right)}
\end{array}
$$

It is interesting that the above set of master integrals is actually redundant since for $i=1,2,3,4, \mathcal{I}_{i}^{n \bar{n}}=\mathcal{I}_{i}^{n n}$. This happens because in certain cases integrals depend on $\alpha_{i}$ and $\beta_{i}$ (or on $n$ and $\bar{n}$ ) in a symmetric way. As an example, consider $\mathcal{I}_{2}^{n \bar{n}}$. We find

$$
\begin{gathered}
\mathcal{I}_{2}^{n \bar{n}}=N_{\epsilon}^{-2} \int \frac{\left[\mathrm{d} k_{1}\right]\left[\mathrm{d} k_{2}\right] \delta\left(1-k_{1} n-k_{2} \bar{n}\right) \delta\left(k_{1} \bar{n}-k_{1} n\right) \theta\left(k_{2} n-k_{2} \bar{n}\right)}{\left(k_{12} n\right)} \\
\stackrel{n \leftrightarrow \bar{n}}{=} N_{\epsilon}^{-2} \int \frac{\left[\mathrm{d} k_{1}\right]\left[\mathrm{d} k_{2}\right] \delta\left(1-k_{1} \bar{n}-k_{2} n\right) \delta\left(k_{1} n-k_{1} \bar{n}\right) \theta\left(k_{2} \bar{n}-k_{2} n\right)}{\left(k_{12} \bar{n}\right)} \\
\stackrel{\left.k_{1} n-k_{1} \bar{n}\right)}{=} N_{\epsilon}^{-2} \int \frac{\left[\mathrm{d} k_{1}\right]\left[\mathrm{d} k_{2}\right] \delta\left(1-k_{1} n-k_{2} n\right) \delta\left(k_{1} \bar{n}-k_{1} n\right) \theta\left(k_{2} \bar{n}-k_{2} n\right)}{\left(k_{12} \bar{n}\right)}=\mathcal{I}_{2}^{n n} .
\end{gathered}
$$

Another interesting feature of the above set of master integrals is that only three master integrals $I_{5 \ldots 7}$ contain two $\theta$-functions; all these integrals correspond to the $n \bar{n}$ configuration. For all other master integrals, either one or both $\theta$-functions are replaced by $\delta$-functions; these integrals are simpler to compute than the original ones.

To understand why there are no $n n$ master integrals with two $\theta$-functions, we note that homogeneous parts of IBP relations are unaffected by $\theta$-functions; hence, by solving homogeneous parts of the IBP relations we should find master integrals that would be present if all $\theta$-functions are removed from the integrand. It is then easy to see that, in the case of $n n$ integrals, removal of $\theta$-functions from the integrand leads to scaleless integrals since the jettiness constraint only depends on $\beta_{1,2}$ in this case.

\subsection{Computation of master integrals}

Having discussed the reduction to master integrals, in this section we explain how to compute the master integrals that appear in eqs. (4.17), (4.18). Since the NNLO soft function is required for the computation of N3LO soft function, we will compute $S_{N A}^{2 g}$ through weight six.

We begin by calculating the four integrals that are needed to describe the $n n$ configuration. To this end, we combine the phase-space parameterization in eq. (2.5) and the Sudakov parametrization of the phase-space element of gluon $i$

$$
\left[\mathrm{d} k_{i}\right]=\frac{\Omega^{(d-2)}}{4(2 \pi)^{d-1}} \mathrm{~d} \alpha_{i} \mathrm{~d} \beta_{i}\left(\alpha_{i} \beta_{i}\right)^{-\epsilon} \frac{\mathrm{d} \Omega_{i, \perp}^{(d-2)}}{\Omega^{(d-2)}}, \quad \alpha_{i}, \beta_{i} \in[0, \infty),
$$


to write

$$
\begin{aligned}
\mathcal{I}_{1}^{n n} & =\int \mathrm{d} \Phi_{\delta \delta}^{n n}=\int \prod_{i=1}^{2} \mathrm{~d} \alpha_{i} \mathrm{~d} \beta_{i}\left(\alpha_{i} \beta_{i}\right)^{-\epsilon} \delta\left(1-\beta_{12}\right) \delta\left(\alpha_{1}-\beta_{1}\right) \delta\left(\alpha_{2}-\beta_{2}\right) \\
& =\int_{0}^{1} \mathrm{~d} \beta_{1} \mathrm{~d} \beta_{2}\left(\beta_{1} \beta_{2}\right)^{-2 \epsilon} \delta\left(1-\beta_{12}\right)=\frac{\Gamma^{2}(1-2 \epsilon)}{\Gamma(2-4 \epsilon)}
\end{aligned}
$$

where we have used $\beta_{12}=\beta_{1}+\beta_{2}$.

The computation of $\mathcal{I}_{2}^{n n}$ proceeds in a similar way. We find

$$
\begin{aligned}
\mathcal{I}_{2}^{n n} & =\int \frac{\mathrm{d} \Phi_{\delta \theta}^{n n}}{\left(k_{12} \bar{n}\right)}=\int_{0}^{\infty} \mathrm{d} \alpha_{2} \mathrm{~d} \beta_{1} \mathrm{~d} \beta_{2} \frac{\left(\alpha_{2} \beta_{1}^{2} \beta_{2}\right)^{-\epsilon} \delta\left(1-\beta_{12}\right) \theta\left(\alpha_{2}-\beta_{2}\right)}{\beta_{1}+\alpha_{2}} \\
\stackrel{\alpha_{2} \rightarrow \beta_{2} / \xi_{2}}{=} & \int_{0}^{1} \mathrm{~d} \xi_{2} \int_{0}^{1} \mathrm{~d} \beta_{1} \frac{\beta_{1}^{-2 \epsilon}\left(1-\beta_{1}\right)^{1-2 \epsilon} \xi_{2}^{\epsilon-1}}{1-\beta_{1}\left(1-\xi_{2}\right)} \\
= & \frac{\Gamma(1-2 \epsilon) \Gamma(2-2 \epsilon)}{\epsilon \Gamma(3-4 \epsilon)}{ }_{3} F_{2}[\{1,1,1-2 \epsilon\},\{3-4 \epsilon, 1+\epsilon\} ; 1] .
\end{aligned}
$$

The integral $\mathcal{I}_{3}^{n n}$ involves the scalar product of the two gluon momenta. To facilitate its computation, we write

$$
\mathcal{I}_{3}^{n n}=\int \frac{\mathrm{d} \Phi_{\delta \theta}^{n n}}{\left(k_{1} k_{2}\right)\left(k_{2} \bar{n}\right)} \stackrel{\alpha_{2} \rightarrow \beta_{2} / \xi_{2}}{=} \int \frac{\mathrm{d} \Omega_{12}^{(d-2)}}{\left[\Omega^{(d-2)}\right]^{2}} \int \frac{\mathrm{d} \xi_{2} \mathrm{~d} \beta_{1} \xi_{2}^{\epsilon}\left[\beta_{1}\left(1-\beta_{1}\right)\right]^{-1-2 \epsilon}}{\left[\xi_{2}+1-2 \sqrt{\xi_{2}} \cos \varphi_{12}\right]},
$$

where $\mathrm{d} \Omega_{12}^{(d-2)}=\mathrm{d} \Omega_{1}^{(d-2)} \mathrm{d} \Omega_{2}^{(d-2)}$ and $\varphi_{12}$ is the relative angle between transverse components of $k_{1}$ and $k_{2}$. To integrate over this angle, we introduce a new variable $\eta$ defined as

$$
\eta=\frac{1-\cos \varphi_{12}}{2}
$$

and write

$$
\mathrm{d} \Omega_{12}^{(d-2)}=2 \mathrm{~d} \Omega^{(d-2)} \mathrm{d} \Omega^{(d-3)} \mathrm{d} \eta[4 \eta(1-\eta)]^{-1 / 2-\epsilon} .
$$

We integrate over $\eta$ using the formula

$$
2 \frac{\Omega^{d-3}}{\Omega^{(d-2)}} \int_{0}^{1} \mathrm{~d} \eta \frac{[4 \eta(1-\eta)]^{-1 / 2-\epsilon}}{\left[\xi_{2}+1-2 \sqrt{\xi_{2}}(1-2 \eta)\right]}=\frac{{ }_{2} F_{1}\left[\{1,1 / 2-\epsilon\},\{1-2 \epsilon\} ; \frac{-4 \sqrt{\xi_{2}}}{\left(1-\sqrt{\xi_{2}}\right)^{2}}\right]}{\left(1-\sqrt{\xi_{2}}\right)^{2}},
$$

apply the following hypergeometric identity ${ }^{8}$

$$
{ }_{2} F_{1}[\{a, b\},\{2 b\} ; z]=\left(1-\frac{z}{2}\right)^{-a}{ }_{2} F_{1}\left[\left\{\frac{a}{2}, \frac{a+1}{2}\right\},\left\{b+\frac{1}{2}\right\} ; \frac{z^{2}}{4(1-z / 2)^{2}}\right],
$$

and obtain

$$
\mathcal{I}_{3}^{n n}=-\frac{2 \Gamma^{2}(1-2 \epsilon)}{\epsilon(1+\epsilon) \Gamma(1-4 \epsilon)}{ }_{3} F_{2}[\{1,1+\epsilon, 1+\epsilon\},\{1-\epsilon, 2+\epsilon\} ; 1] .
$$

\footnotetext{
${ }^{8}$ See, e.g. eq. (15.3.16) in ref. [57].
} 
Following similar steps, we derive

$$
\mathcal{I}_{4}^{n n}=\int \frac{\mathrm{d} \Phi_{\delta \theta}^{n n}}{\left(k_{1} k_{2}\right)\left(k_{2} n\right)\left(k_{12} \bar{n}\right)}=-2 \frac{\Gamma^{2}(1-2 \epsilon)}{\epsilon \Gamma(1-4 \epsilon)} \int_{0}^{1} \mathrm{~d} \xi_{2} \xi_{2}^{-1-\epsilon}\left(1-\xi_{2}\right)^{-1-2 \epsilon} \mathcal{X}_{4}\left(\xi_{2}\right)
$$

where the function $\mathcal{X}_{4}$ reads

$$
\mathcal{X}_{4}\left(\xi_{2}\right)={ }_{2} F_{1}\left[\{-1-4 \epsilon,-2 \epsilon\},\{-4 \epsilon\} ; 1-\xi_{2}\right]{ }_{2} F_{1}\left[\{-\epsilon,-2 \epsilon\},\{1-\epsilon\} ; \xi_{2}\right]
$$

We note that the integral in eq. (4.29) diverges logarithmically at the integration boundaries $\xi_{2}=0,1$ but the function $\mathcal{X}_{4}\left(\xi_{2}\right)$ is regular at these points

$$
\mathcal{X}_{4}(0)=\frac{\Gamma(1-4 \epsilon) \Gamma(1+2 \epsilon)}{2 \Gamma(1-2 \epsilon)}, \quad \mathcal{X}_{4}(1)=\frac{\Gamma(1-\epsilon) \Gamma(1+2 \epsilon)}{\Gamma(1+\epsilon)} .
$$

Hence, we can compute the master integral $\mathcal{I}_{4}^{n n}$ by subtracting divergent contributions at endpoints and adding them back. Specifically, we write

$$
\begin{aligned}
\mathcal{I}_{4}^{n n}= & -2 \frac{\Gamma^{2}(1-2 \epsilon)}{\epsilon \Gamma(1-4 \epsilon)}\left\{\mathcal{X}_{4}(0) \int_{0}^{1} \mathrm{~d} \xi_{2} \xi_{2}^{-1-\epsilon}+\mathcal{X}_{4}(1) \int_{0}^{1} \mathrm{~d} \xi_{2}\left(1-\xi_{2}\right)^{-1-2 \epsilon}\right. \\
& \left.+\int_{0}^{1} \mathrm{~d} \xi_{2}\left[\xi_{2}^{-1-\epsilon}\left(1-\xi_{2}\right)^{-1-2 \epsilon} \mathcal{X}_{4}\left(\xi_{2}\right)-\xi_{2}^{-1-\epsilon} \mathcal{X}_{4}(0)-\left(1-\xi_{2}\right)^{-1-2 \epsilon} \mathcal{X}_{4}(1)\right]\right\} .
\end{aligned}
$$

The first two integrals on the r.h.s of eq. (4.32) are trivial. The last integral is regular in the integration domain $\xi_{2} \in[0,1]$ and can be computed after expansion in $\epsilon$. We construct such an expansion using the package HypExp [58] and use the program HyperInt [59] to integrate the result over $\xi_{2}$. We arrive at

$$
\mathcal{I}_{4}^{n n}=\frac{2}{\epsilon^{2}}+\frac{\pi^{2}}{3}-\frac{17 \pi^{4} \epsilon^{2}}{90}+\epsilon^{3}\left[-6 \pi^{2} \zeta_{3}-26 \zeta_{5}\right]-\epsilon^{4}\left[\frac{193 \pi^{6}}{810}+64 \zeta_{3}^{2}\right]+\mathcal{O}\left(\epsilon^{5}\right),
$$

where we have discarded contributions of weight seven and higher.

It remains to compute three additional integrals for the $n \bar{n}$ configuration. We begin with $\mathcal{I}_{5}^{n \bar{n}}$ and change variables $\alpha_{1}=\beta_{1} / \xi_{1}, \beta_{2}=\alpha_{2} / \xi_{2}$. We find

$$
\begin{aligned}
\mathcal{I}_{5}^{n \bar{n}}= & \int \frac{\mathrm{d} \Phi_{\theta \theta}^{n \bar{n}}}{\left(k_{12} n\right)\left(k_{12} \bar{n}\right)} \\
= & \frac{2 \Gamma^{2}(2-2 \epsilon)}{\Gamma(4-4 \epsilon)} \\
& \times \int_{0}^{1} \mathrm{~d} \xi_{1} \int_{0}^{1} \mathrm{~d} \xi_{2} \frac{\left(1-\xi_{1}\right)\left(\xi_{1} \xi_{2}\right)^{-1+\epsilon}}{1-\xi_{1} \xi_{2}}{ }_{2} F_{1}\left[\{1,2-2 \epsilon\},\{4-4 \epsilon\} ; 1-\xi_{1}\right] \\
= & \frac{2 \Gamma^{2}(2-2 \epsilon)}{\epsilon \Gamma(4-4 \epsilon)} \int_{0}^{1} \mathrm{~d} \xi_{1}\left(1-\xi_{1}\right) \xi_{1}^{-1+\epsilon} \mathcal{X}_{5}\left(\xi_{1}\right),
\end{aligned}
$$

where

$$
\mathcal{X}_{5}\left(\xi_{1}\right)={ }_{2} F_{1}\left[\{1, \epsilon\},\{1+\epsilon\} ; \xi_{1}\right]_{2} F_{1}\left[\{1,2-2 \epsilon\},\{4-4 \epsilon\} ; 1-\xi_{1}\right] .
$$


We subtract the (only) logarithmic singularity at $\xi_{1}=0$ and obtain after the integration

$$
\begin{aligned}
\mathcal{I}_{5}^{n \bar{n}}= & \frac{1}{\epsilon^{2}}+\frac{2}{\epsilon}+\left[2+\frac{\pi^{2}}{6}\right]+\epsilon\left[2 \zeta_{3}-8+\pi^{2}\right]+\epsilon^{2}\left[16 \zeta_{3}-64+4 \pi^{2}+\frac{\pi^{4}}{9}\right] \\
& +\epsilon^{3}\left[64 \zeta_{3}-\frac{2 \pi^{2} \zeta_{3}}{3}+30 \zeta_{5}-256+\frac{32 \pi^{2}}{3}+\frac{26 \pi^{4}}{45}\right] \\
& +\epsilon^{4}\left[128 \zeta_{3}+4 \pi^{2} \zeta_{3}+8 \zeta_{3}^{2}+60 \zeta_{5}-512+\frac{92 \pi^{4}}{45}+\frac{44 \pi^{6}}{945}\right]+\mathcal{O}\left(\epsilon^{5}\right) .
\end{aligned}
$$

Following the same steps as described above, we find the result for $\mathcal{I}_{6}^{n \bar{n}}$

$$
\mathcal{I}_{6}^{n \bar{n}}=-\frac{2 \Gamma^{2}(1-2 \epsilon)}{\epsilon(1+\epsilon)^{2} \Gamma(1-4 \epsilon)}{ }_{4} F_{3}[\{1,1+\epsilon, 1+\epsilon, 1+\epsilon\},\{1-\epsilon, 2+\epsilon, 2+\epsilon\} ; 1] .
$$

Finally, for the integral $\mathcal{I}_{7}^{n \bar{n}}$ we obtain

$$
\begin{aligned}
\mathcal{I}_{7}^{n \bar{n}}= & \int \frac{\mathrm{d} \Phi_{\theta \theta}^{n \bar{n}}}{\left(k_{1} k_{2}\right)\left(k_{2} n\right)\left(k_{12} \bar{n}\right)} \\
= & -\frac{\Gamma^{2}(1-2 \epsilon)}{\epsilon(1+\epsilon) \Gamma(1-4 \epsilon)} \int_{0}^{1} \mathrm{~d} \xi_{1} \xi_{1}^{\epsilon} \\
& \times{ }_{2} F_{1}\left[\{1,-2 \epsilon\},\{1-4 \epsilon\} ; 1-\xi_{1}\right]_{3} F_{2}\left[\{1,1+\epsilon, 1+\epsilon\},\{1-\epsilon, 2+\epsilon\} ; \xi_{1}\right] .
\end{aligned}
$$

The $\xi_{1}$-integral is finite; we expand it in powers of $\epsilon$ and integrate using HyperInt. The result reads

$$
\mathcal{I}_{7}^{n \bar{n}}=-\frac{\pi^{2}}{6 \epsilon}+2 \zeta_{3}+\frac{\pi^{4} \epsilon}{12}+\epsilon^{2}\left[\frac{5 \pi^{2} \zeta_{3}}{3}+19 \zeta_{5}\right]+\epsilon^{3}\left[\frac{937 \pi^{6}}{3780}-82 \zeta_{3}^{2}\right]+\mathcal{O}\left(\epsilon^{4}\right) .
$$

This concludes the computation of master integrals required for the calculation of the real-emission contribution to the zero-jettiness soft function at NNLO.

\subsection{Results for the real emission contribution at NNLO}

We use the master integrals computed in section 4.3, insert them into eq. (4.16) and obtain

$$
\begin{aligned}
S_{\mathrm{NA}}^{2 g}=\tau^{-1-4 \epsilon} & \left\{\frac{2}{\epsilon^{3}}+\frac{11}{3 \epsilon^{2}}+\frac{1}{\epsilon}\left[\frac{67}{9}-\pi^{2}\right]+\left[\frac{404}{27}-\frac{11 \pi^{2}}{9}-18 \zeta_{3}\right]\right. \\
+ & \epsilon\left[\frac{2140}{81}+\frac{67 \pi^{2}}{9}-\frac{59 \pi^{4}}{90}-\frac{220 \zeta_{3}}{3}\right]+\epsilon^{2}\left[\frac{12416}{243}-\frac{368 \pi^{2}}{81}\right. \\
& \left.-\frac{143 \pi^{4}}{45}+268 \zeta_{3}+4 \pi^{2} \zeta_{3}-182 \zeta_{5}\right]+\epsilon^{3}\left[\frac{67528}{729}+\frac{2416 \pi^{2}}{81}\right. \\
& \left.\left.+\frac{469 \pi^{4}}{45}-\frac{17 \pi^{6}}{105}-\frac{7864 \zeta_{3}}{27}+\frac{880 \pi^{2} \zeta_{3}}{9}-122 \zeta_{3}^{2}-\frac{6248 \zeta_{5}}{3}\right]+\mathcal{O}\left(\epsilon^{4}\right)\right\} .
\end{aligned}
$$

The result agrees with the one derived earlier in ref. [24]. ${ }^{9}$

\footnotetext{
${ }^{9}$ We note that it also agrees with the results of refs. [21, 22] which were computed through weight four. Partial results through weight six have been obtained in ref. [60].
} 
To summarize, we have shown that by constructing the integration-by-parts identities for phase-space integrals with Heaviside functions it is possible to express the real emission contribution to the zero-jettiness NNLO soft function through seven master integrals. The majority of these integrals needs to be computed by integrating over a simplified phase space with all or some $\theta$-functions replaced by the $\delta$-functions. This simplification is very striking in case of the $n n$ kinematic configuration where we find that no master integrals with two $\theta$-functions need to be computed. As we discussed earlier, this interesting feature can be readily understood if IBP technology is applied to phase-space integrals with Heaviside functions.

\section{Testing the method with some N3LO contributions to the zero-jettiness soft function}

It appears from the discussion in the previous section that it is useful to construct IBP relations for integrals with Heaviside functions. However, given an enormous growth in computational complexity with increase in the perturbative order, it is important to check this statement by considering a more complex example. Given our interest in the N3LO QCD contribution to the zero-jettiness soft function, it is natural to check if modified IBP relations can be constructed and used to compute it.

To this end, in this section we consider the maximally non-abelian part of the realemission contribution to the soft function with all gluons emitted to the same hemisphere. We define it as follows

$$
S_{n n n}^{3 g}=\tau^{-1-6 \epsilon} \int \mathrm{d} \Phi_{\theta \theta \theta}^{n n n} \omega_{n \bar{n}}^{(3)}\left(k_{1}, k_{2}, k_{3}\right),
$$

where the function $\omega_{n \bar{n}}^{(3)}\left(k_{1}, k_{2}, k_{3}\right)$ in eq. (5.1) reads [61]

$$
\begin{gathered}
\omega_{n \bar{n}}^{(3)}\left(k_{1}, k_{2}, k_{3}\right)=\sum_{t \in\{a, b, c, d\}} \omega_{n \bar{n}}^{(3), t}\left(k_{1}, k_{2}, k_{3}\right) \\
\omega_{n \bar{n}}^{(3), t}=\left[\overline{\mathcal{S}}_{n \bar{n}}^{(t)}\left(k_{1}, k_{2}, k_{3}\right)+\overline{\mathcal{S}}_{\bar{n} n}^{(t)}\left(k_{1}, k_{2}, k_{3}\right)-\overline{\mathcal{S}}_{n n}^{(t)}\left(k_{1}, k_{2}, k_{3}\right)-\overline{\mathcal{S}}_{\bar{n} \bar{n}}^{(t)}\left(k_{1}, k_{2}, k_{3}\right)\right] \\
+ \text { permutations }\left\{k_{1}, k_{2}, k_{3}\right\} .
\end{gathered}
$$

In eq. (5.3), "permutations $\left\{k_{1}, k_{2}, k_{3}\right\}$ " describes all possible permutations of the gluon momenta $k_{i}$. The four terms $\overline{\mathcal{S}}_{i k}^{(t)}, t=a, b, c, d$ in eq. (5.3) are contributions to the soft eikonal function that are ordered according to the structure of their collinear singularities [61].

A simple generalization of the discussion in section 3 implies that, in order to set up IBP relations for integrals that appear in $S_{n n n}^{3 g}$, we require eight distinct classes of integrals. They correspond to integrations over the following phase spaces $I_{\theta \theta \theta} \sim \int \mathrm{d} \Phi_{\theta \theta \theta}^{n n n}$, $I_{\theta \theta \delta} \sim \int \mathrm{d} \Phi_{\theta \theta \delta}^{n n n}, I_{\theta \delta \delta} \sim \int \mathrm{d} \Phi_{\theta \delta \delta}^{n n n}, I_{\delta \delta \delta} \sim \int \mathrm{d} \Phi_{\delta \delta \delta}^{n n n}$ and four more cases where the ordering of $\theta$ - and $\delta$-functions differs from the above examples.

Similar to the two-gluon case, these classes of integrals possess hierarchical structure that we rely upon when solving the integration-by-parts identities. Indeed, a closed set of IBP 
relations can be derived for $I_{\delta \delta \delta}$ using reverse unitarity. On the other hand, IBP identities for $I_{\theta \delta \delta}$ involve $I_{\delta \delta \delta}$, and IBP identities for $I_{\theta \theta \delta}$ involve $I_{\theta \delta \delta}$ and $I_{\delta \delta \delta}$. Finally, integration-byparts identities for $I_{\theta \theta \theta}$ make use of all other integrals with $\theta$ - and $\delta$-functions. We illustrate how this approach applies to various N3LO QCD contributions to the zero-jettiness soft function in the next sections.

\subsection{The $\omega_{n \bar{n}}^{(3), a}$ contribution}

The simplest contribution to consider is an integral of $\omega_{n \bar{n}}^{(3), a}$. According to eq. (5.3), this function is constructed from the function $\overline{\mathcal{S}}_{i k}^{(a)}$, which reads $[61]^{10}$

$$
\begin{aligned}
& \overline{\mathcal{S}}_{i k}^{(a)}\left(q_{1}, q_{2}, q_{3}\right)=\frac{31}{144} \frac{\left(p_{i} p_{k}\right)^{3}}{p_{i} q_{1} p_{k} q_{1} p_{i} q_{2} p_{k} q_{2} p_{i} q_{3} p_{k} q_{3}} \\
& \quad+\frac{\left(p_{i} p_{k}\right)^{3}}{32 p_{k} q_{12} p_{k} q_{3} p_{i} q_{1} p_{i} q_{3}}\left(\frac{1}{p_{k} q_{1}}-\frac{1}{p_{k} q_{2}}\right)\left(\frac{6}{p_{i} q_{12}}+\frac{p_{i}\left(q_{3}-q_{1}\right)}{p_{i} q_{13} p_{i} q_{2}}\right) \\
& \quad+\frac{\left(p_{i} p_{k}\right)^{3}}{288 p_{k} q_{123} p_{i} q_{123}}\left(\frac{1}{p_{k} q_{1}}-\frac{1}{p_{k} q_{2}}\right) \times\left\{\frac{2}{p_{i} q_{1}}\left(\frac{1}{p_{i} q_{3}}-\frac{3}{p_{i} q_{12}}\right)\left(\frac{1}{p_{k} q_{3}}-\frac{3}{p_{k} q_{12}}\right)\right. \\
& \left.\quad+\left(\frac{1}{p_{i} q_{2}}-\frac{3}{p_{i} q_{13}}\right)\left(\frac{1}{p_{i} q_{1}}-\frac{1}{p_{i} q_{3}}\right)\left(\frac{1}{p_{k} q_{3}}-\frac{3}{p_{k} q_{12}}\right)\right\},
\end{aligned}
$$

where $p_{i}=n, p_{k}=\bar{n}$ and $q_{i}=k_{i}, i=1,2,3$ in our notations. As can be easily checked, $\overline{\mathcal{S}}_{i k}^{(a)}$ is not singular when any of the two gluons become collinear to each other. This feature reduces the number of scalar products that appear in the denominators of that function, making integration over three-gluon phase space simpler.

Applying integration-by-parts identities, we find that the integral of $\omega_{n \bar{n}}^{(3), a}$ over the phase space $\mathrm{d} \Phi_{\theta \theta \theta}^{n n n}$ can be expressed through six master integrals. The result of the reduction reads

$$
\begin{aligned}
& \int \mathrm{d} \Phi_{\theta \theta \theta}^{n n n} \omega_{n \bar{n}}^{(3), a}\left(k_{1}, k_{2}, k_{3}\right) \\
= & \frac{1}{(1-4 \epsilon)(1-5 \epsilon)}\left\{\left[\frac{182}{5 \epsilon^{5}}-\frac{3392}{5 \epsilon^{4}}+\frac{23268}{5 \epsilon^{3}}-\frac{69432}{5 \epsilon^{2}}+\frac{75728}{5 \epsilon}\right] I_{1}\right. \\
& +\left[\frac{8}{5 \epsilon^{3}}-\frac{72}{5 \epsilon^{2}}+\frac{32}{\epsilon}\right] I_{2}+\left[-\frac{112}{5 \epsilon^{4}}+\frac{2016}{5 \epsilon^{3}}-\frac{13328}{5 \epsilon^{2}}+\frac{38304}{5 \epsilon}-8064\right] I_{3} \\
& +\left[-\frac{4}{\epsilon^{4}}+\frac{356}{5 \epsilon^{3}}-\frac{2248}{5 \epsilon^{2}}+\frac{5968}{5 \epsilon}-\frac{5664}{5}\right] I_{4}+\left[-\frac{8}{5 \epsilon^{3}}+\frac{104}{5 \epsilon^{2}}-\frac{448}{5 \epsilon}+128\right] I_{5} \\
& \left.+\left[-\frac{36}{5 \epsilon^{4}}+\frac{648}{5 \epsilon^{3}}-\frac{4428}{5 \epsilon^{2}}+2592 \epsilon+\frac{14328}{5 \epsilon}-\frac{22032}{5}\right] I_{6}\right\} .
\end{aligned}
$$

Definitions of the six master integrals are given in appendix A, eq. (A.1). However, it is useful to emphasize at this point that none of these integrals contains three $\theta$-functions. We have explained why this happens when discussing the NNLO contribution to the soft function in section 4 .

Calculation of master integrals that appear in eq. (5.5) is rather straightforward. As an example, consider a phase-space integral $I_{1}$ with three $\delta$-functions. It reads

$$
I_{1}=\int \mathrm{d} \Phi_{\delta \delta \delta}^{n n n} .
$$

\footnotetext{
${ }^{10}$ We note that we take the emitters $i, j$ to be massless.
} 
Since the integrand does not depend on the relative orientation of the three partons in the transverse plane, we can integrate over $\mathrm{d} \Omega_{i, \perp}^{(d-2)}$ for $i=1,2,3$. We then remove all $\delta\left(\alpha_{i}-\beta_{i}\right)$-functions by integrating over $\alpha_{i}, i=1,2,3$, and find

$$
I_{1}=\int \prod_{i=1}^{3} \mathrm{~d} \beta_{i} \beta_{i}^{-2 \epsilon} \delta\left(1-\beta_{123}\right)=\frac{\Gamma^{3}(1-2 \epsilon)}{\Gamma(3-6 \epsilon)} .
$$

For a less trivial example, consider the integral $I_{5}$. It reads

$$
I_{5}=\int \frac{\mathrm{d} \Phi_{\delta \delta \theta}^{n n n}}{\left(k_{13} n\right)\left(k_{123} \bar{n}\right)} .
$$

To compute this integral, we remove two $\delta$-functions by integrating over $\alpha_{1,2}$ and then integrate over $\beta_{1}$ to remove $\delta\left(1-\beta_{123}\right)$. Then, writing $\beta_{2}=\left(1-\beta_{3}\right) \xi$, we arrive at

$$
I_{5}=\int \mathrm{d} \alpha_{3} \mathrm{~d} \beta_{3} \alpha_{3}^{-\epsilon} \beta_{3}^{-\epsilon} \theta\left(\alpha_{3}-\beta_{3}\right) \frac{\left(1-\beta_{3}\right)^{1-4 \epsilon}}{1-\beta_{3}+\alpha_{3}} \int_{0}^{1} \frac{\mathrm{d} \xi \xi^{-2 \epsilon}(1-\xi)^{-2 \epsilon}}{1-\left(1-\beta_{3}\right) \xi} .
$$

It is straightforward to express the integrals over $\xi$ and $\alpha_{3}$ through hypergeometric functions. The result reads

$$
I_{5}=\frac{\Gamma^{2}(1-2 \epsilon)}{\epsilon \Gamma(2-4 \epsilon)} \int_{0}^{1} \mathrm{~d} u(1-u)^{1-2 \epsilon} u^{1-4 \epsilon}{ }_{2} F_{1}[\{1,1-2 \epsilon\},\{2-4 \epsilon\} ; u]_{2} F_{1}[\{1,1\},\{1+\epsilon\} ; u],
$$

where we introduced a new variable $u=1-\beta_{3}$. Although both hypergeometric functions in eq. (5.10) are singular at $u=1$, this singularity is made integrable by an explicit factor $(1-u)^{1-2 \epsilon}$ in the integrand. Hence, we can directly expand the integrand in eq. (5.10) in powers of $\epsilon$ and integrate over $u$ using HyperInt [59]. We find

$$
\begin{aligned}
I_{5}= & \frac{1}{\epsilon}+8+\epsilon\left(2 \zeta_{3}-\frac{7 \pi^{2}}{6}+46\right)+\epsilon^{2}\left(-26 \zeta_{3}+\frac{83 \pi^{4}}{360}-\frac{23 \pi^{2}}{3}+216\right) \\
& +\epsilon^{3}\left(-\frac{1}{6} 89 \pi^{2} \zeta_{3}-180 \zeta_{3}+\frac{585 \zeta_{5}}{2}+\frac{17 \pi^{4}}{180}-\frac{86 \pi^{2}}{3}+776\right) \\
& +\epsilon^{4}\left(-271 \zeta_{3}^{2}-\frac{89 \pi^{2} \zeta_{3}}{3}-536 \zeta_{3}+729 \zeta_{5}+\frac{7739 \pi^{6}}{7560}+\frac{4 \pi^{2}}{3}-\frac{149 \pi^{4}}{90}+1024\right)
\end{aligned}
$$

where we retained all contributions through weight six.

The results for all other integrals which appear in eq. (5.5) can be obtained along similar lines; they are given in the supplementary material. Using them in eq. (5.5), we obtain a remarkably simple result for the integral of $\omega_{n \bar{n}}^{(3), a}$ in the $n n n$ configuration

$$
\begin{aligned}
\int \mathrm{d} \Phi_{\theta \theta \theta}^{n n n} \omega_{n \bar{n}}^{(3), a}\left(k_{1}, k_{2}, k_{3}\right)= & \frac{8}{\epsilon^{5}}-\frac{20 \pi^{2}}{\epsilon^{3}}-\frac{584 \zeta_{3}}{\epsilon^{2}}-\frac{86 \pi^{4}}{15 \epsilon}+\left(1472 \pi^{2} \zeta_{3}-12480 \zeta_{5}\right) \\
& +\epsilon\left(22288 \zeta_{3}^{2}-\frac{3796 \pi^{6}}{945}\right),
\end{aligned}
$$

showing the usefulness of applying integration-by-parts technology to phase-space integrals with Heaviside functions. 


\subsection{The $\omega_{n \bar{n}}^{(3), b}$ contribution}

We consider the second contribution to the zero-jettiness soft function given by an integral of the function $\omega_{n \bar{n}}^{(3), b}$. This function is constructed from the function $\overline{\mathcal{S}}_{i k}^{(b)}$, which reads [61]

$$
\begin{aligned}
\overline{\mathcal{S}}_{i k}^{(b)}= & \frac{1}{16 q_{1} q_{2} p_{i} q_{12}}\left\{\frac{7 p_{i} p_{k}}{p_{i} q_{1} p_{i} q_{3} p_{k} q_{2} p_{k} q_{3}}\left(-p_{i} p_{k} p_{i} q_{12}\right)\right. \\
& \left.+\frac{\left(p_{i} p_{k}\right)^{2}}{p_{i} q_{3}}\left[\frac{12 p_{i}\left(q_{1}-q_{2}\right)}{p_{k} q_{12} p_{i} q_{1} p_{k} q_{3}}+\frac{1}{p_{k} q_{13}}\left(\frac{1}{p_{k} q_{3}}-\frac{1}{p_{k} q_{1}}\right)\left(3+\frac{p_{i} q_{1}}{p_{i} q_{2}}-\frac{2 p_{k} q_{1}}{p_{k} q_{2}}\right)\right]\right\} \\
& +\frac{1}{48 q_{1} q_{2} p_{k} q_{123}}\left\{\frac{3}{p_{k} q_{1} p_{i} q_{2}}\left(\frac{1}{p_{k} q_{12}}-\frac{1}{p_{k} q_{3}}\right)\left(\frac{p_{i} p_{k}}{p_{i} q_{3}}\right)\left(p_{i} p_{k} p_{k} q_{12}\right)\right. \\
& \left.+3\left(\frac{1}{p_{k} q_{3}}-\frac{1}{p_{k} q_{12}}\right)\left(p_{i} p_{k} p_{k} q_{12}\right)\left[\frac{p_{i} p_{k}}{p_{i} q_{23} p_{k} q_{1}}\left(\frac{1}{p_{i} q_{2}}-\frac{1}{p_{i} q_{3}}\right)\right]\right\} \\
& +\frac{p_{i} p_{k}}{48 q_{1} q_{2} p_{k} q_{123} p_{i} q_{123}}\left(\frac{1}{p_{i} q_{12}}-\frac{1}{p_{i} q_{3}}\right)\left(2 p_{i} p_{k} p_{k}\left(q_{2}-q_{1}\right)\right) \\
& \times\left[\left(\frac{1}{p_{k} q_{3}}-\frac{3}{p_{k} q_{12}}\right)\left(\frac{1}{p_{k} q_{1}}-\frac{1}{p_{k} q_{2}}\right)+\left(\frac{1}{p_{k} q_{2}}-\frac{3}{p_{k} q_{13}}\right)\left(\frac{1}{p_{k} q_{1}}-\frac{1}{p_{k} q_{3}}\right)\right],
\end{aligned}
$$

where $p_{i}=n, p_{k}=\bar{n}$ and $q_{i}=k_{i}, i=1,2,3$ in our notations. At variance with $\overline{\mathcal{S}}_{i k}^{(a)}$, the function $\overline{\mathcal{S}}_{i k}^{(b)}$ contains a scalar product of two gluon momenta $q_{1} q_{2}$ which causes a collinear singularity in the limit $\boldsymbol{q}_{1} \| \boldsymbol{q}_{2}$ and makes it more difficult to integrate the function $\omega_{n \bar{n}}^{(3), b}$ compared to the discussion in the previous section. However, there is no reason to expect that things may work differently for this contribution.

We have, therefore, proceeded as before and performed a reduction to master integrals. To check our results numerically, we derived Mellin-Barnes (MB) representations for relevant integrals [62] and used computer packages developed in refs. [63, 64] to compute them numerically. Comparing numerical and analytic results, we have found that reduction to master integrals produces wrong results because not all integrals that appear in the integration-by-parts identities in the course of the reduction are regulated dimensionally.

It is interesting to point out that a) integrals that appear in the function $\omega_{n \bar{n}}^{(3), b}\left(k_{1}, k_{2}, k_{3}\right)$ are well-defined in dimensional regularization and b) all master integrals that appear in the expression for the amplitude obtained after the reduction do not exhibit singularities that cannot be regulated dimensionally. This means that the failure of dimensional regularization is very well hidden in the internal dynamic of the reduction process and, therefore, hard to detect.

To remedy this situation, we introduced an analytic regulator in addition to the dimensional one. The analytic regulator is introduced in such a way that the phase-space element is multiplied by a factor

$$
\mathrm{d} \Phi_{f f f}^{n n n} \rightarrow \mathrm{d} \Phi_{f f f}^{n n n}\left(k_{1} n\right)^{\nu}\left(k_{2} n\right)^{\nu}\left(k_{3} n\right)^{\nu} .
$$

This modification changes the integration-by-parts equations making them $\nu$-dependent and, therefore, significantly more complicated. ${ }^{11}$

\footnotetext{
${ }^{11}$ To illustrate increase in complexity related to the introduction of the regulator, we mention that the size of the reduction tables grew from $300 \mathrm{~KB}$ (without the regulator) to $64 \mathrm{MB}$ (with the regulator).
} 
However, the main steps that we have described earlier in connection with deriving IBP equations and establishing a hierarchy of integrals is not affected by the analytic regulator.

Having performed the reduction for finite values of $\nu$ and $\epsilon$, we have found that the integral of $\omega_{n \bar{n}}^{(3), b}\left(k_{1}, k_{2}, k_{3}\right)$ can be written in a remarkably simple form which, however, demonstrates very clearly why the analytic regulator is needed. We obtain

$$
\int \mathrm{d} \Phi_{\theta \theta \theta}^{n n n}\left(k_{1} n\right)^{\nu}\left(k_{2} n\right)^{\nu}\left(k_{3} n\right)^{\nu} \omega_{n \bar{n}}^{(3), b}\left(k_{1}, k_{2}, k_{3}\right)=\sum_{i=1}^{23} C_{i}^{(b)}(\epsilon, \nu) J_{i}(\epsilon, \nu) .
$$

As indicated in eq. (5.15), both the reduction coefficients and the integrals $J_{1, ., 23}$ are functions of $\nu$ but, studying the $\nu \rightarrow 0$ limit of eq. (5.15), we find that for $i=1,2, . ., 22$ the following holds

$$
\lim _{\nu \rightarrow 0} C_{i}(\epsilon, \nu)=C_{i}(\epsilon), \quad \lim _{\nu \rightarrow 0} J_{i}(\epsilon, \nu)=I_{i} .
$$

The integrals $I_{1, . ., 6}$ have been already discussed in the previous section and $I_{7, \ldots, 22}$ are another sixteen integrals that can be evaluated at $\nu=0$. These integrals are given in eq. (A.2).

The last integral, $J_{23}(\epsilon, \nu)$, has a $1 / \nu$ pole but $C_{23}(\epsilon, \nu)$ is proportional to $\nu$. Therefore, $C_{23}(\epsilon, \nu) J_{23}(\epsilon, \nu)$ produces a finite result in the $\nu \rightarrow 0$ limit that, however, is completely missed if the reduction at $\nu=0$ is performed.

Following this discussion and taking the $\nu \rightarrow 0$ limit where appropriate, we write the result of the reduction as follows

$$
\begin{aligned}
& \int \mathrm{d} \Phi_{\theta \theta \theta}^{n n n} \omega_{n \bar{n}}^{(3), b}\left(k_{1}, k_{2}, k_{3}\right)= \\
& \frac{\left(10249456 \epsilon^{5}-6479980 \epsilon^{4}+713856 \epsilon^{3}+268429 \epsilon^{2}-67966 \epsilon+4287\right)}{200 \epsilon^{5}(2 \epsilon-1)(4 \epsilon-1)(5 \epsilon-1)} I_{1} \\
& +\frac{7}{5 \epsilon^{3}} I_{2}-\frac{38\left(504 \epsilon^{3}-270 \epsilon^{2}+37 \epsilon-1\right)}{5 \epsilon^{4}(2 \epsilon-1)} I_{3} \\
& -\frac{\left(4260192 \epsilon^{5}-3531008 \epsilon^{4}+674380 \epsilon^{3}+124140 \epsilon^{2}-49897 \epsilon+3923\right)}{50 \epsilon^{4}\left(40 \epsilon^{3}-38 \epsilon^{2}+11 \epsilon-1\right)} I_{4} \\
& +\frac{\left(704 \epsilon^{2}-280 \epsilon+26\right)}{5 \epsilon^{3}-10 \epsilon^{4}} I_{5}+\frac{9\left(9288 \epsilon^{4}-7308 \epsilon^{3}+858 \epsilon^{2}+347 \epsilon-55\right)}{5 \epsilon^{4}(4 \epsilon-1)} I_{6}-\frac{237}{4 \epsilon^{2}} I_{7} \\
& -\frac{6(4 \epsilon+1)}{\epsilon^{3}} I_{8}+\frac{10}{3 \epsilon^{2}} I_{9}-\frac{10}{\epsilon^{2}} I_{10}+\frac{6}{\epsilon^{2}} I_{11}+\frac{6}{5 \epsilon^{2}} I_{12}-\frac{6}{5 \epsilon^{2}} I_{13}+\frac{18}{\epsilon^{2}} I_{14}-\frac{9}{5 \epsilon^{2}} I_{15} \\
& +\frac{(14-84 \epsilon)}{5 \epsilon^{2}} I_{16}+\frac{12}{5 \epsilon} I_{17}+\frac{93}{5 \epsilon} I_{18}-\frac{12}{\epsilon} I_{19}-\frac{39}{\epsilon} I_{20}+\frac{22(4 \epsilon-1)}{\epsilon^{2}} I_{21} \\
& -\frac{7}{\epsilon} I_{22}-\frac{4}{3 \epsilon^{2}}\left[\lim _{\nu \rightarrow 0} \nu J_{\nu}(\epsilon, \nu)\right],
\end{aligned}
$$

where we renamed $J_{23}$ to $J_{\nu}$.

As we already mentioned the integrals $I_{1 . .22}$ that appear in the above expression can be found in appendix A, eqs. (A.1), (A.2). The integral that is singular in the $\nu \rightarrow 0$ limit is defined as follows

$$
J_{\nu}(\epsilon, \nu)=\int \frac{\mathrm{d} \Phi_{\theta \delta \theta}^{n n n}\left(k_{1} n\right)^{\nu}\left(k_{2} n\right)^{\nu}\left(k_{3} n\right)^{\nu}}{\left(k_{1} k_{3}\right)\left(k_{1} n\right)\left(k_{12} \bar{n}\right)\left(k_{3} \bar{n}\right)} .
$$

We will now explain how this "pathological" integral can be computed. 
To integrate over the relative azimuthal angle between $\vec{k}_{1, \perp}$ and $\vec{k}_{3, \perp}$, we use the following formula

$$
\begin{array}{r}
\int \frac{\mathrm{d} \Omega^{(d-2)}}{\Omega^{(d-2)}} \frac{1}{k_{i} k_{j}}=2\left\{\frac{\theta\left(\alpha_{i} \beta_{j}-\alpha_{j} \beta_{i}\right)}{\alpha_{i} \beta_{j}}{ }_{2} F_{1}\left[\{1,1+\epsilon\},\{1-\epsilon\} ; \frac{\alpha_{j} \beta_{i}}{\alpha_{i} \beta_{j}}\right]\right. \\
\left.+\frac{\theta\left(\alpha_{j} \beta_{i}-\alpha_{i} \beta_{j}\right)}{\alpha_{j} \beta_{i}}{ }_{2} F_{1}\left[\{1,1+\epsilon\},\{1-\epsilon\} ; \frac{\alpha_{i} \beta_{j}}{\alpha_{j} \beta_{i}}\right]\right\},
\end{array}
$$

where $i=1$ and $j=3$. To proceed further, we change variables $\alpha_{1,3} \rightarrow \beta_{1,3} / \xi_{1,3}$ and obtain the following integral representation

$$
\begin{aligned}
J_{\nu}= & 2 \int \prod_{i=1}^{3} \mathrm{~d} \beta_{i} \beta_{i}^{-2 \epsilon+\nu} \delta\left(1-\beta_{123}\right) \frac{\mathrm{d} \xi_{1} \mathrm{~d} \xi_{3}\left(\xi_{1} \xi_{3}\right)^{\epsilon-1}}{\beta_{1} \beta_{3}\left(\beta_{1}+\beta_{2} \xi_{1}\right)}\left\{\xi_{3} \theta\left(\xi_{1}-\xi_{3}\right)\right. \\
& \left.\times{ }_{2} F_{1}\left[\{1,1+\epsilon\},\{1-\epsilon\} ; \frac{\xi_{3}}{\xi_{1}}\right]+\xi_{1} \theta\left(\xi_{3}-\xi_{1}\right){ }_{2} F_{1}\left[\{1,1+\epsilon\},\{1-\epsilon\} ; \frac{\xi_{1}}{\xi_{3}}\right]\right\} .
\end{aligned}
$$

We make a further change of variables, $\xi_{3}=r f$ and $\xi_{1}=f$ in the first term in the square brackets and $\xi_{3}=f$ and $\xi_{1}=r f$ in the second one. We find

$$
\begin{aligned}
J_{\nu}= & 2 \int \prod_{i=1}^{3} \mathrm{~d} \beta_{i} \beta_{i}^{-2 \epsilon+\nu} \delta\left(1-\beta_{123}\right) \mathrm{d} r \mathrm{~d} f r^{\epsilon} f^{2 \epsilon}{ }_{2} F_{1}[\{1,1+\epsilon\},\{1-\epsilon\} ; r] \\
& \times \frac{1}{\beta_{1} \beta_{3}}\left(\frac{1}{\left(\beta_{1}+\beta_{2} f\right)}+\frac{1}{\left(\beta_{1}+\beta_{2} f r\right)}\right)
\end{aligned}
$$

It is convenient to write $\beta_{1}=x(1-y), \beta_{2}=x y$ and $\beta_{3}=1-x, 0<x, y<1$. Upon doing that, we find that integrations over $x$ and $y$ can be readily performed. We obtain

$$
J_{\nu}=2 \frac{\Gamma(-4 \epsilon+2 \nu) \Gamma(-2 \epsilon+\nu)}{\Gamma(-6 \epsilon+3 \nu)} \frac{\Gamma(1-2 \epsilon+\nu) \Gamma(-2 \epsilon+\nu)}{\Gamma(1-4 \epsilon+2 \nu)} \tilde{J}_{\nu}
$$

where

$$
\begin{aligned}
\tilde{J}_{\nu}= & \int \mathrm{d} f \mathrm{~d} r f^{2 \epsilon} r^{\epsilon}\left[{ }_{2} F_{1}[\{1,1-2 \epsilon+\nu\},\{1-4 \epsilon+2 \nu\} ; 1-f]\right. \\
& \left.+{ }_{2} F_{1}[\{1,1-2 \epsilon+\nu\},\{1-4 \epsilon+2 \nu\} ; 1-r f]\right]{ }_{2} F_{1}[\{1,1+\epsilon\},\{1-\epsilon\} ; r] .
\end{aligned}
$$

The two hypergeometric functions in the square brackets in the last equation can be conveniently re-written using the following identity

$$
\begin{aligned}
{ }_{2} F_{1}[\{1,1-2 \epsilon+\nu\},\{1-4 \epsilon+2 \nu\} ; z] & \\
& =(1-z)^{-1-2 \epsilon+\nu}{ }_{2} F_{1}[\{-4 \epsilon+2 \nu,-2 \epsilon+\nu\},\{1-4 \epsilon+2 \nu\} ; z]
\end{aligned}
$$


Inserting eq. (5.24) into eq. (5.23), we obtain

$$
\begin{aligned}
\tilde{J}_{\nu}= & \int_{0}^{1} \mathrm{~d} f \mathrm{~d} r f^{\nu-1} r^{\epsilon}\left\{{ }_{2} F_{1}[\{-4 \epsilon+2 \nu,-2 \epsilon+\nu\},\{1-4 \epsilon+2 \nu\} ; 1-f]\right. \\
& \left.+r^{-2 \epsilon+\nu-1}{ }_{2} F_{1}[\{-4 \epsilon+2 \nu,-2 \epsilon+\nu\},\{1-4 \epsilon+2 \nu\} ; 1-r f]\right\} \\
& \times{ }_{2} F_{1}[\{1,1+\epsilon\},\{1-\epsilon\} ; r] .
\end{aligned}
$$

From the above equation we readily see how the $1 / \nu$-singularity appears; it is generated by the singularity at $f=0$ in eq. (5.25) which is not regulated dimensionally.

Although we can compute $\tilde{J}_{\nu}$ by expanding it in Laurent series in $\epsilon$ and $\nu$, we only require the $1 / \nu$ singularity of this integral. We therefore compute the residue at $f=0$ and find

$$
\begin{aligned}
& \left.\tilde{J}_{\nu}\right|_{\nu \rightarrow 0}=\frac{{ }_{2} F_{1}[\{-4 \epsilon,-2 \epsilon\},\{1-4 \epsilon\} ; 1]}{\nu} \int_{0}^{1} \mathrm{~d} r r_{2}^{\epsilon} F_{1}[\{1,1+\epsilon\},\{1-\epsilon\} ; r]\left[1+r^{-2 \epsilon-1}\right] \\
& =\frac{{ }_{2} F_{1}[\{-4 \epsilon,-2 \epsilon\},\{1-4 \epsilon\} ; 1]}{\nu}\left(\frac{\Gamma(1+\epsilon)}{\Gamma(2+\epsilon)}{ }_{3} F_{2}[\{1,1+\epsilon, 1+\epsilon\},\{1-\epsilon, 2+\epsilon\} ; 1]\right. \\
& \left.+\frac{\Gamma(-\epsilon)}{\Gamma(1-\epsilon)}{ }_{3} F_{2}[\{1,1+\epsilon,-\epsilon\},\{1-\epsilon, 1-\epsilon\} ; 1]\right) .
\end{aligned}
$$

We now use eqs. (5.22), (5.26), expand the result in $\epsilon$ and obtain

$$
\begin{aligned}
\lim _{\nu \rightarrow 0}\left(\nu J_{\nu}\right)= & -\frac{3}{2 \epsilon^{3}}+\frac{\pi^{2}}{\epsilon}+75 \zeta_{3}+\frac{77 \pi^{4} \epsilon}{20}+\epsilon^{2}\left(2025 \zeta_{5}-50 \pi^{2} \zeta_{3}\right) \\
& +\epsilon^{3}\left(\frac{1787 \pi^{6}}{210}-1875 \zeta_{3}^{2}\right)+\mathcal{O}\left(\epsilon^{4}\right) .
\end{aligned}
$$

Calculation of the master integrals $I_{1,2, \ldots, 22}$ required for computing the integral of the function $\omega_{n \bar{n}}^{(3), b}$ proceeds in full analogy to the above case and for this reason we do not discuss it here. Finally, if we use the reduction to master integrals eq. (5.17) and explicit results for the master integrals that can be found in the supplementary material, we obtain the following result for the integral of the function $\omega_{n \bar{n}}^{(3), b}$ in the $n n n$ configuration

$$
\begin{aligned}
\int \mathrm{d} \Phi_{\theta \theta \theta}^{n n n} \omega_{n \bar{n}}^{(3), b}\left(k_{1}, k_{2}, k_{3}\right)= & \frac{8}{\epsilon^{5}}+\frac{32}{\epsilon^{4}}+\frac{1}{\epsilon^{3}}\left(64-\frac{41 \pi^{2}}{3}\right) \\
& +\frac{1}{\epsilon^{2}}\left(128-64 \pi^{2}-774 \zeta_{3}\right)+\frac{1}{\epsilon}\left(256-128 \pi^{2}-\frac{581 \pi^{4}}{10}-2144 \zeta_{3}\right) \\
+ & \left(512-256 \pi^{2}-\frac{1688 \pi^{4}}{15}-4288 \zeta_{3}+\frac{1306 \pi^{2} \zeta_{3}}{3}-28770 \zeta_{5}\right) \\
+ & \epsilon\left(1024-512 \pi^{2}-\frac{3376 \pi^{4}}{15}-\frac{616 \pi^{6}}{5}-8576 \zeta_{3}+2304 \pi^{2} \zeta_{3}\right. \\
& \left.+19480 \zeta_{3}^{2}-68736 \zeta_{5}\right) .
\end{aligned}
$$


Again, we see that, apart from an unexpected (and interesting) complication related to the need to introduce an analytic regulator, it is beneficial to make use of the IBP identities to compute non-trivial contributions to the zero-jettiness soft function at N3LO.

\subsection{The $\omega_{n \bar{n}}^{(3), c}$ contribution}

The third contribution to the zero-jettiness soft function is associated with the integral of the function $\omega_{n \bar{n}}^{(3), c}$. Similar to the other two contributions, we construct $\omega_{n \bar{n}}^{(3), c}$ from the function $\overline{\mathcal{S}}_{i k}^{(c)}$, which reads [61]

$$
\begin{aligned}
\overline{\mathcal{S}}_{i k}^{(c)}= & \frac{1}{8\left(q_{1} q_{2}\right)^{2} p_{i} q_{12} p_{k} q_{3}}\left\{( ( 4 - d ) p _ { i } q _ { 1 } + d p _ { i } q _ { 2 } ) \left[\frac{p_{i} p_{k} p_{k} q_{1}}{2 p_{k} q_{123} p_{i} q_{123}}\left(\frac{p_{k} q_{3}}{p_{k} q_{12}}-1\right)\left(\frac{p_{i} q_{12}}{p_{i} q_{3}}-1\right)\right.\right. \\
& \left.+\frac{p_{i} p_{k}}{p_{i} q_{3}}\left(\frac{p_{i} q_{1}}{p_{i} q_{12}}-\frac{3}{2} \frac{p_{k} q_{1}}{p_{k} q_{12}}\right)\right] \\
& \left.+\frac{p_{i} p_{k}}{p_{i} q_{123}}\left(\frac{1}{p_{i} q_{3}}-\frac{1}{p_{i} q_{12}}\right)\left((4-d)\left(p_{i} q_{1}\right)^{2}+d p_{i} q_{1} p_{i} q_{2}\right)\right\} \\
& +\frac{1}{32 q_{1} q_{2} q_{1} q_{3} p_{i} q_{12}}\left\{\frac { p _ { i } p _ { k } } { p _ { k } q _ { 2 } } \left[\frac{4 p_{k}\left(q_{2}-q_{1}\right)}{p_{k} q_{3}}+\frac{2 p_{i} q_{12}}{p_{i} q_{3}}+2 \frac{p_{i} q_{2} p_{i} q_{3}+p_{i} q_{1} p_{i} q_{123}}{p_{i} q_{13} p_{i} q_{3}}\right.\right. \\
& \left.+\frac{1}{p_{k} q_{13} p_{i} q_{3}}\left(p_{i} q_{1} p_{k}\left(5 q_{1}-8 q_{2}+2 q_{3}\right)-3 p_{i} q_{2} p_{k} q_{3}+4 p_{i} p_{k} q_{2} q_{3}\right)\right] \\
& +\frac{2}{p_{i} q_{123} p_{k} q_{3}}\left(\frac{1}{p_{i} q_{2}}-\frac{1}{p_{i} q_{13}}\right)\left(p_{i} p_{k} p_{i} q_{12} p_{i} q_{13}\right) \\
& +\frac{2}{p_{k} q_{123} p_{k} q_{3}}\left(\frac{1}{p_{k} q_{13}}-\frac{1}{p_{k} q_{2}}\right)\left[2 p_{i} p_{k} p_{k} q_{13} p_{k}\left(q_{2}-q_{1}\right)\right]+\frac{1}{p_{k} q_{123} p_{i} q_{123}}\left(1-\frac{p_{i} q_{12}}{p_{i} q_{3}}\right) \\
& \left.\times\left(\frac{1}{p_{k} q_{13}}-\frac{1}{p_{k} q_{2}}\right)\left[4\left(p_{i} p_{k}\right)^{2} q_{2} q_{3}+p_{i} p_{k}\left(p_{i} q_{1} p_{k}\left(5 q_{1}-8 q_{2}+2 q_{3}\right)-3 p_{i} q_{2} p_{k} q_{3}\right)\right]\right\},
\end{aligned}
$$

where $p_{i}=n, p_{k}=\bar{n}$ and $q_{i}=k_{i}, i=1,2,3$ in our notations. At variance with the functions $\overline{\mathcal{S}}_{i k}^{(a, b)}$, the function $\overline{\mathcal{S}}_{i k}^{(c)}$ contains propagators $1 /\left(q_{1} q_{3}\right)$ and $1 /\left(q_{2} q_{3}\right)$ and, therefore, exhibits a more complicated singularity structure. However, no additional issues arise when modified IBP relations are constructed and used to reduce required integrals, so that working with the analytic regulator introduced in the previous section, see eq. (5.14), suffices.

Performing the reduction to master integrals and taking the $\nu \rightarrow 0$ limit where appropriate, we obtain

$$
\begin{aligned}
& \int \mathrm{d} \Phi_{\theta \theta \theta}^{n n n} \omega_{n \bar{n}}^{(3), c}\left(k_{1}, k_{2}, k_{3}\right)= \\
& \left(-\frac{5152}{675 \epsilon^{5}}+\frac{60883}{1350 \epsilon^{4}}+\frac{2218663}{5400 \epsilon^{3}}-\frac{33423797}{10800 \epsilon^{2}}-\frac{49850253233 \epsilon}{466560}+\frac{44313583}{12960 \epsilon}+\frac{40023347}{15552}\right) I_{1} \\
& +\left(-\frac{262}{135 \epsilon^{3}}+\frac{8}{135 \epsilon^{2}}-\frac{1280 \epsilon}{9}-\frac{16}{9 \epsilon}+\frac{160}{9}\right) I_{2}+\left(\frac{2242}{135 \epsilon^{4}}-\frac{23954}{135 \epsilon^{3}}+\frac{74967872 \epsilon^{2}}{1215}\right. \\
& \left.+\frac{14596}{45 \epsilon^{2}}+\frac{2845312 \epsilon}{405}+\frac{69832}{45 \epsilon}-\frac{733168}{135}\right) I_{3}+\left(-\frac{47683}{675 \epsilon^{4}}+\frac{27427}{25 \epsilon^{3}}-\frac{7899529}{1350 \epsilon^{2}}\right. \\
& \left.+\frac{11094485353 \epsilon^{2}}{12960}-\frac{119650151 \epsilon}{2160}+\frac{7137263}{900 \epsilon}+\frac{5243129}{360}\right) I_{4}+\left(\frac{334}{135 \epsilon^{3}}-\frac{600703 \epsilon^{2}}{81}\right.
\end{aligned}
$$




$$
\begin{aligned}
& \left.-\frac{794}{45 \epsilon^{2}}+\frac{38854 \epsilon}{27}+\frac{5036}{135 \epsilon}-\frac{2024}{9}\right) I_{5}+\left(-\frac{1202}{15 \epsilon^{4}}+\frac{4022}{3 \epsilon^{3}}+\frac{6077660177 \epsilon^{2}}{6480}-\frac{121631}{15 \epsilon^{2}}\right. \\
& \left.-\frac{76894303 \epsilon}{1080}+\frac{170291}{10 \epsilon}+\frac{534641}{180}\right) I_{6}+\left(-3211 \epsilon^{4}+1409 \epsilon^{3}-151 \epsilon^{2}-\frac{9}{2 \epsilon^{2}}+119 \epsilon\right. \\
& \left.+\frac{3}{2 \epsilon}+44\right) I_{7}+\left(\frac{16640 \epsilon^{4}}{3}-\frac{3968 \epsilon^{3}}{3}+\frac{1088 \epsilon^{2}}{3}-\frac{14}{3 \epsilon^{2}}-\frac{224 \epsilon}{3}-\frac{8}{3 \epsilon}+\frac{80}{3}\right) I_{9}+\frac{65}{9 \epsilon^{2}} I_{10} \\
& +\left(-\frac{430971 \epsilon^{4}}{32}+\frac{238589 \epsilon^{3}}{48}-\frac{21817 \epsilon^{2}}{24}-\frac{47}{9 \epsilon^{2}}+\frac{1527 \epsilon}{4}+\frac{337}{9 \epsilon}+\frac{47}{6}\right) I_{11}+\left(\frac{1613075 \epsilon^{4}}{96}\right. \\
& \left.-\frac{118055 \epsilon^{3}}{48}+\frac{8195 \epsilon^{2}}{24}-\frac{13}{15 \epsilon^{2}}-\frac{515 \epsilon}{12}+\frac{25}{6}\right)\left[I_{12}-I_{13}\right]+\left(-\frac{127360 \epsilon^{4}}{3}+\frac{18560 \epsilon^{3}}{3}\right. \\
& \left.-\frac{2560 \epsilon^{2}}{3}-\frac{578}{45 \epsilon^{2}}+\frac{320 \epsilon}{3}+\frac{16}{45 \epsilon}-\frac{32}{3}\right) I_{14}+\left(-\frac{377080 \epsilon^{4}}{9}+\frac{54680 \epsilon^{3}}{9}-\frac{7480 \epsilon^{2}}{9}\right. \\
& \left.+\frac{19}{15 \epsilon^{2}}+\frac{920 \epsilon}{9}+\frac{8}{45 \epsilon}-\frac{88}{9}\right) I_{15}+\left(-\frac{110384 \epsilon^{3}}{3}+\frac{21808 \epsilon^{2}}{3}-\frac{92}{15 \epsilon^{2}}-\frac{4496 \epsilon}{3}\right. \\
& \left.+\frac{212}{15 \epsilon}+\frac{832}{3}\right) I_{16}+\left(-\frac{1075877 \epsilon^{5}}{16}+\frac{114497 \epsilon^{4}}{8}-\frac{10397 \epsilon^{3}}{4}+\frac{1217 \epsilon^{2}}{2}-17 \epsilon\right. \\
& \left.+\frac{68}{5 \epsilon}+14\right) I_{17}+\left(\frac{1111653 \epsilon^{5}}{16}-\frac{127713 \epsilon^{4}}{8}+\frac{9933 \epsilon^{3}}{4}-\frac{1593 \epsilon^{2}}{2}-87 \epsilon+\frac{12}{5 \epsilon}-36\right) I_{18} \\
& +\left(\frac{2546205 \epsilon^{5}}{16}-\frac{275865 \epsilon^{4}}{8}+\frac{22005 \epsilon^{3}}{4}-\frac{3465 \epsilon^{2}}{2}-15 \epsilon+\frac{18}{\epsilon}-150\right) I_{19} \\
& +\left(-172 \epsilon^{3}+44 \epsilon^{2}+32 \epsilon+\frac{2}{\epsilon}-14\right) I_{20}+\left(64 \epsilon^{3}+32 \epsilon^{2}-\frac{4}{3 \epsilon^{2}}+16 \epsilon+\frac{4}{3 \epsilon}+8\right) I_{21} \\
& +\left(1968 \epsilon^{3}-320 \epsilon^{2}+\frac{172 \epsilon}{3}+\frac{40}{9 \epsilon}-\frac{68}{9}\right) I_{22}+\left(-312 \epsilon^{3}+\frac{151}{60 \epsilon^{3}}-\frac{4532 \epsilon^{2}}{3}+\frac{73}{6 \epsilon^{2}}\right. \\
& \left.-270 \epsilon-\frac{69}{10 \epsilon}-\frac{353}{3}\right) I_{23}+\left(3840 \epsilon^{4}-672 \epsilon^{3}+96 \epsilon^{2}-24 \epsilon-\frac{2}{\epsilon}\right) I_{24}+\left(69719 \epsilon^{4}\right. \\
& \left.-9413 \epsilon^{3}+2419 \epsilon^{2}+\frac{1}{3 \epsilon^{2}}-107 \epsilon-\frac{125}{18 \epsilon}+\frac{920}{9}\right) I_{25}+\left(\frac{83680 \epsilon^{4}}{3}-\frac{13280 \epsilon^{3}}{3}+\frac{2080 \epsilon^{2}}{3}\right. \\
& \left.+\frac{4}{45 \epsilon^{2}}-\frac{320 \epsilon}{3}-\frac{104}{45 \epsilon}+16\right) I_{26}+\left(-96 \epsilon^{4}-48 \epsilon^{3}-24 \epsilon^{2}-12 \epsilon-\frac{3}{\epsilon}-6\right) I_{27} \\
& +\left(\frac{4480 \epsilon^{5}}{3}+\frac{2240 \epsilon^{4}}{3}+\frac{1120 \epsilon^{3}}{3}+\frac{560 \epsilon^{2}}{3}+\frac{4}{3 \epsilon^{2}}+\frac{280 \epsilon}{3}+\frac{91}{6 \epsilon}+\frac{140}{3}\right) I_{28} \\
& +\left(117120 \epsilon^{5}-19200 \epsilon^{4}+3360 \epsilon^{3}-480 \epsilon^{2}-\frac{2}{\epsilon^{2}}+120 \epsilon+\frac{2}{3 \epsilon}\right) I_{29}+\left(34560 \epsilon^{5}\right. \\
& \left.-5760 \epsilon^{4}+960 \epsilon^{3}-160 \epsilon^{2}+\frac{80 \epsilon}{3}+\frac{20}{9 \epsilon}-\frac{40}{9}\right) I_{30}+\left(-96576 \epsilon^{5}+15216 \epsilon^{4}\right. \\
& \left.-2976 \epsilon^{3}+276 \epsilon^{2}+\frac{2}{3 \epsilon^{2}}-156 \epsilon-\frac{9}{\epsilon}-29\right) I_{31}+\left(-\frac{40832 \epsilon^{5}}{3}+\frac{7232 \epsilon^{4}}{3}-\frac{992 \epsilon^{3}}{3}\right. \\
& \left.+\frac{272 \epsilon^{2}}{3}+\frac{7}{18 \epsilon^{2}}+\frac{8 \epsilon}{3}+\frac{29}{18 \epsilon}+\frac{76}{9}\right) I_{32}+\left(-5728 \epsilon^{4}+1048 \epsilon^{3}-128 \epsilon^{2}-\frac{37}{18 \epsilon^{2}}\right. \\
& \left.+\frac{134 \epsilon}{3}-\frac{9}{2 \epsilon}+\frac{38}{9}\right) I_{33}+\left(\frac{93152 \epsilon^{5}}{3}-\frac{15632 \epsilon^{4}}{3}+\frac{2552 \epsilon^{3}}{3}-\frac{452 \epsilon^{2}}{3}-\frac{7}{18 \epsilon^{2}}+\frac{62 \epsilon}{3}-\frac{1}{6 \epsilon}\right.
\end{aligned}
$$




$$
\begin{aligned}
& \left.-\frac{17}{3}\right) I_{34}+\left(34695 \epsilon^{5}-5805 \epsilon^{4}+975 \epsilon^{3}-165 \epsilon^{2}+\frac{85 \epsilon}{3}+\frac{5}{3 \epsilon}-5\right)\left[I_{35}-I_{36}+I_{37}\right] \\
& +\frac{4}{3 \epsilon}\left[I_{38}-I_{39}\right]+\left(-\frac{16640 \epsilon^{4}}{3}+\frac{3968 \epsilon^{3}}{3}-\frac{1088 \epsilon^{2}}{3}+\frac{8}{3 \epsilon^{2}}+\frac{224 \epsilon}{3}+\frac{8}{3 \epsilon}\right. \\
& \left.-\frac{80}{3}\right)\left[\lim _{\nu \rightarrow 0} \nu J_{\nu}\right]+\mathcal{O}\left(\epsilon^{2}\right) .
\end{aligned}
$$

All the master integrals $I_{1, . ., 39}$ that appear in eq. (5.30) are computed at $\nu=0$; their definition can be found in appendix A, eqs. (A.1), (A.2), (A.3). The only $\nu$-dependent integral $J_{\nu}$ present in eq. (5.30) is the same integral which we have seen (and computed) in the previous section.

In principle, computation of the $I$-integrals that appear in the calculation of the case "c" does not differ from what we already discussed for cases "a" and "b". However, integrals with two separate scalar products of parton's four-momenta are significantly more complicated than what we have discussed so far and, to illustrate this point, we will discuss how one of them can be computed.

We consider integral $I_{30}$ defined as

$$
I_{30}=\int \frac{\mathrm{d} \Phi_{\theta \delta \theta}^{n n n}}{\left(k_{1} k_{2}\right)\left(k_{1} k_{3}\right)\left(k_{12} n\right)\left(k_{13} \bar{n}\right)} .
$$

We can use eq. (5.19) to perform integration over azimuthal angles of $k_{2}$ and $k_{3}$. We note, however, that the momentum $k_{2}$ is special because the $\delta$-function in the definition of $I_{30}$ implies that $\alpha_{2}=\beta_{2}$. This condition removes one of the two hypergeometric functions in eq. (5.19). Integrating over azimuthal angles and changing variables $\alpha_{1,3}=\beta_{1,3} / \xi_{1,3}$, we arrive at the following representation for $I_{30}$

$$
\begin{aligned}
I_{30}= & 4 \int \prod_{i=1}^{3} \mathrm{~d} \beta_{i} \beta_{i}^{-2 \epsilon-1} \delta\left(1-\beta_{123}\right) \frac{\mathrm{d} \xi_{1} \mathrm{~d} \xi_{3} \xi_{1}^{\epsilon} \xi_{3}^{\epsilon-1} \beta_{3}}{\left(\beta_{1}+\beta_{2}\right)\left(\beta_{1} \xi_{3}+\beta_{3} \xi_{1}\right)} \\
& \times{ }_{2} F_{1}\left[\{1,1+\epsilon\},\{1-\epsilon\} ; \xi_{1}\right]\left[\xi_{1} \theta\left(\xi_{3}-\xi_{1}\right)_{2} F_{1}\left[\{1,1+\epsilon\},\{1-\epsilon\} ; \frac{\xi_{1}}{\xi_{3}}\right]\right. \\
& \left.+\xi_{3} \theta\left(\xi_{1}-\xi_{3}\right)_{2} F_{1}\left[\{1,1+\epsilon\},\{1-\epsilon\} ; \frac{\xi_{3}}{\xi_{1}}\right]\right]
\end{aligned}
$$

As the next step, we remove $\delta\left(1-\beta_{123}\right)$ by integrating over $\beta_{2}$ and change variables $\left(\beta_{1}, \beta_{3}\right) \rightarrow(x, y)$ according to the following formula $\beta_{1}=x(1-y), \beta_{3}=x y$. Integration over $x$ leads to a hypergeometric function. We obtain

$$
\begin{aligned}
I_{30}= & \frac{4 \Gamma(-4 \epsilon) \Gamma(-2 \epsilon)}{\Gamma(-6 \epsilon)} \int \mathrm{d} y \mathrm{~d} \xi_{1} \mathrm{~d} \xi_{3} \frac{y^{-2 \epsilon}(1-y)^{-2 \epsilon-1} \xi_{1}^{\epsilon} \xi_{3}^{\epsilon}}{(1-y) \xi_{3}+y \xi_{1}} \\
& \times{ }_{2} F_{1}[\{1,-4 \epsilon\},\{-6 \epsilon\} ; y]_{2} F_{1}\left[\{1,1+\epsilon\},\{1-\epsilon\} ; \xi_{1}\right] \\
& \times\left[\frac{\xi_{1}}{\xi_{3}} \theta\left(\xi_{3}-\xi_{1}\right){ }_{2} F_{1}\left[\{1,1+\epsilon\},\{1-\epsilon\} ; \frac{\xi_{1}}{\xi_{3}}\right]+\theta\left(\xi_{1}-\xi_{3}\right){ }_{2} F_{1}\left[\{1,1+\epsilon\},\{1-\epsilon\} ; \frac{\xi_{3}}{\xi_{1}}\right]\right] .
\end{aligned}
$$


The integral naturally splits into two parts. To proceed, we change variables $\xi_{1}=$ $r \xi, \xi_{3}=\xi$, and $\xi_{3}=r \xi, \quad \xi_{1}=\xi$, in the first and the second integral in eq. (5.33), respectively. We also rewrite the hypergeometric functions that appear in square brackets in the above equation using the transformation

$$
{ }_{2} F_{1}[\{a, b\},\{c\} ; z]=(1-z)^{c-a-b}{ }_{2} F_{1}[\{c-a, c-b\},\{c\} ; z] .
$$

We then write

$$
I_{30}=\frac{4 \Gamma(-4 \epsilon) \Gamma(-2 \epsilon)}{\Gamma(-6 \epsilon)}\left(\mathcal{I}_{30}^{(a)}+\mathcal{I}_{30}^{(b)}\right)
$$

where

$$
\begin{aligned}
\mathcal{I}_{30}^{(a)}= & \int \mathrm{d} y \mathrm{~d} \xi \mathrm{d} r \frac{y^{-2 \epsilon}(1-y)^{-4 \epsilon-2} \xi^{2 \epsilon} r^{1+\epsilon}[(1-r)(1-r \xi)]^{-1-2 \epsilon}}{1-y(1-r)} \\
& \times{ }_{2} F_{1}[\{-1-6 \epsilon,-2 \epsilon\},\{-6 \epsilon\} ; y]{ }_{2} F_{1}[\{-\epsilon,-2 \epsilon\},\{1-\epsilon\} ; r \xi] \\
& \times{ }_{2} F_{1}[\{-\epsilon,-2 \epsilon\},\{1-\epsilon\} ; r] .
\end{aligned}
$$

and

$$
\begin{aligned}
\mathcal{I}_{30}^{(b)}= & \int \mathrm{d} y \mathrm{~d} \xi \mathrm{d} r \frac{y^{-2 \epsilon}(1-y)^{-4 \epsilon-2} \xi^{2 \epsilon} r^{\epsilon}(1-r)^{-2 \epsilon-1}}{r+y(1-r)} \\
& \times{ }_{2} F_{1}[\{-1-6 \epsilon,-2 \epsilon\},\{-6 \epsilon\} ; y]{ }_{2} F_{1}[\{1,1+\epsilon\},\{1-\epsilon\} ; \xi] \\
& \times{ }_{2} F_{1}[\{-\epsilon,-2 \epsilon\},\{1-\epsilon\} ; r] .
\end{aligned}
$$

The difficulty with computing these integrals is a power-like singularity at $y=1$. To extract and isolate it, we transform the $y$-dependent hypergeometric function in the following way

$$
\begin{aligned}
{ }_{2} F_{1}[\{-1-6 \epsilon,-2 \epsilon\},\{-6 \epsilon\} ; y]=\frac{\Gamma(-6 \epsilon) \Gamma(1+2 \epsilon)}{\Gamma(-4 \epsilon)} y^{1+6 \epsilon} \\
\quad+(1-y)^{1+2 \epsilon} \frac{\Gamma(-1-2 \epsilon) \Gamma(-6 \epsilon)}{\Gamma(-1-6 \epsilon) \Gamma(-2 \epsilon)}{ }_{2} F_{1}[\{1,-4 \epsilon\},\{2+2 \epsilon\} ; 1-y] .
\end{aligned}
$$

This representation is helpful because the $y$-dependence of the first term in eq. (5.38) is simple so that integration over $y$ can be immediately performed, and the second term in eq. (5.38) leads to integrals with only a logarithmic singularity at $y=1$.

To proceed further, we consider integral $\mathcal{I}_{30}^{(a)}$ and write it as

$$
\mathcal{I}_{30}^{(a)}=\mathcal{I}_{30}^{(a, 1)}+\mathcal{I}_{30}^{(a, 2)},
$$

where the two terms correspond to the two terms on the right hand side of eq. (5.38). To compute $\mathcal{I}_{30}^{(a, 1)}$ we integrate over $y$ and obtain

$$
\int \mathrm{d} y \frac{y^{1+4 \epsilon}(1-y)^{-4 \epsilon-2}}{1-y(1-r)}=\Gamma(2+4 \epsilon) \Gamma(-1-4 \epsilon) r^{-2-4 \epsilon} .
$$


We then use this result in the expression for $\mathcal{I}_{30}^{(a, 1)}$ and write

$$
\begin{aligned}
\mathcal{I}_{30}^{(a, 1)}= & -\frac{\Gamma(2+4 \epsilon) \Gamma(-6 \epsilon) \Gamma(1+2 \epsilon)}{(1+4 \epsilon)} \int \mathrm{d} \xi \mathrm{d} r \xi^{2 \epsilon} r^{-1-3 \epsilon}(1-r)^{-2 \epsilon-1}(1-\xi r)^{-2 \epsilon-1} \\
& \times{ }_{2} F_{1}[\{-\epsilon,-2 \epsilon\},\{1-\epsilon\} ; r \xi]{ }_{2} F_{1}[\{-\epsilon,-2 \epsilon\},\{1-\epsilon\} ; r] .
\end{aligned}
$$

This integral has a singularity at $r=0$ and another (overlapping) singularity at $r=1, \xi=1$. The two singularities can be separated by multiplying the integrand with $1=(1-r)+r$. The first term in the sum removes the $r=1$ singularity. Since the $r=0$ singularity does not overlap with any other singularity, it can be easily extracted. On the contrary, the $r=1$ singularity overlaps with $\xi=1$ singularity and for this reason a slightly more sophisticated treatment is needed. To this end, we subtract the product of hypergeometric functions at $r=1$ and add it back. When the difference

$$
\begin{aligned}
& { }_{2} F_{1}[\{-\epsilon,-2 \epsilon\},\{1-\epsilon\} ; r \xi]{ }_{2} F_{1}[\{-\epsilon,-2 \epsilon\},\{1-\epsilon\} ; r] \\
& -{ }_{2} F_{1}[\{-\epsilon,-2 \epsilon\},\{1-\epsilon\} ; \xi]{ }_{2} F_{1}[\{-\epsilon,-2 \epsilon\},\{1-\epsilon\} ; 1],
\end{aligned}
$$

is used in the integrand, it becomes non-singular at $r=1$, so that we can expand it in $\epsilon$ and integrate. On the other hand, hypergeometric functions in the subtraction term do not depend on $r$ so that integration over $r$ becomes straightforward. The resulting one-dimensional integration over $\xi$ contains a logarithmic singularity at $\xi=1$ that can be easily isolated and extracted. Putting everything together, we find

$$
\begin{aligned}
\mathcal{I}_{30}^{(a, 1)}= & -\frac{1}{18 \epsilon^{2}}+\frac{1}{48 \epsilon^{3}}+\frac{1}{\epsilon}\left(\frac{5}{18}+\frac{\pi^{2}}{6}\right)+\frac{43 \zeta_{3}}{12}+\frac{5 \pi^{2}}{108}-\frac{25}{18} \\
& +\epsilon\left(\frac{61 \zeta_{3}}{6}+\frac{437 \pi^{4}}{540}+\frac{125}{18}-\frac{13 \pi^{2}}{108}\right)+\epsilon^{2}\left(\frac{89 \pi^{2} \zeta_{3}}{9}-\frac{85 \zeta_{3}}{2}+\frac{845 \zeta_{5}}{4}+\frac{209 \pi^{4}}{162}\right. \\
& \left.+\frac{17 \pi^{2}}{108}-\frac{625}{18}\right)+\epsilon^{3}\left(-32 \zeta_{3}^{2}+\frac{304 \pi^{2} \zeta_{3}}{9}+\frac{1075 \zeta_{3}}{6}+\frac{3059 \zeta_{5}}{6}+\frac{57637 \pi^{6}}{17010}\right. \\
& \left.+\frac{107 \pi^{2}}{108}+\frac{3125}{18}-\frac{2194 \pi^{4}}{405}\right) .
\end{aligned}
$$

The computation of $\mathcal{I}_{30}^{(a, 2)}$ proceeds in the following way. First, we observe that in this case there are two singularities, $y=1$ and $r=1$. We note that the latter overlaps with the $\xi=1$ singularity. To disentangle overlapping singularities, we replace

$$
\begin{aligned}
& \frac{r^{1+\epsilon}}{1-y(1-r)}{ }_{2} F_{1}[\{-\epsilon,-2 \epsilon\},\{1-\epsilon\} ; \xi r]_{2} F_{1}[\{-\epsilon,-2 \epsilon\},\{1-\epsilon\} ; r] \rightarrow \\
& \left.{ }_{2} F_{1}[\{-\epsilon,-2 \epsilon\},\{1-\epsilon\} ; \xi]\right]_{2} F_{1}[\{-\epsilon,-2 \epsilon\},\{1-\epsilon\} ; 1],
\end{aligned}
$$

in eq. (5.36) and add the difference of the two terms back. In the difference, the $r=1$ singularity is regulated, so that one only needs to extract a logarithmic $y=1$ singularity. To compute the contribution of the subtraction term (the r.h.s. of eq. (5.44)), we can integrate over $y$ and over $r$ to obtain yet another hypergeometric function of $\xi$. The resulting integral 
over $\xi$ has a logarithmic singularity at $\xi=1$ which can be easily extracted. We obtain

$$
\begin{aligned}
\mathcal{I}_{30}^{(a, 2)}= & -\frac{1}{16 \epsilon^{3}}-\frac{1}{4 \epsilon^{2}}+\frac{1}{\epsilon}\left(1-\frac{\pi^{2}}{4}\right)-\frac{15 \zeta_{3}}{4}-\frac{2 \pi^{2}}{3}-4+\epsilon\left(-23 \zeta_{3}+\frac{8 \pi^{2}}{3}\right. \\
& \left.-\frac{23 \pi^{4}}{36}+16\right)+\epsilon^{2}\left(\frac{23 \pi^{2} \zeta_{3}}{6}+104 \zeta_{3}-\frac{595 \zeta_{5}}{4}-\frac{32 \pi^{2}}{3}-\frac{47 \pi^{4}}{30}-64\right) \\
& +\epsilon^{3}\left(249 \zeta_{3}^{2}+\frac{2 \pi^{2} \zeta_{3}}{3}-476 \zeta_{3}-577 \zeta_{5}+\frac{67 \pi^{4}}{10}+\frac{128 \pi^{2}}{3}-\frac{4756 \pi^{6}}{2835}+256\right) .
\end{aligned}
$$

Computation of the integral $\mathcal{I}_{30}^{(b)}$ proceeds in a similar way except that the integration over $\xi$ can be performed right away. We find

$$
\begin{aligned}
\mathcal{I}_{30}^{(b)}= & \frac{1}{1+2 \epsilon}{ }_{3} F_{2}[\{1,1+\epsilon, 1+2 \epsilon\},\{1-\epsilon, 2+2 \epsilon\} ; 1] \\
& \times \int \mathrm{d} y \mathrm{~d} r \frac{y^{-2 \epsilon}(1-y)^{-4 \epsilon-2} r^{\epsilon}(1-r)^{-2 \epsilon-1}}{r+y(1-r)} \\
& \times{ }_{2} F_{1}[\{-1-6 \epsilon,-2 \epsilon\},\{-6 \epsilon\} ; y]{ }_{2} F_{1}[\{-\epsilon,-2 \epsilon\},\{1-\epsilon\} ; r] .
\end{aligned}
$$

We then rewrite ${ }_{2} F_{1}[\{-1-6 \epsilon,-2 \epsilon\},\{-6 \epsilon\} ; y]$ using eq. (5.38) and integrate the two terms that appear in that equation separately. This integration is relatively straightforward since integration of the first term in eq. (5.38) leads to yet another ${ }_{3} F_{2}$-function and integration of the second term does not require resolution of any overlapping singularities.

Putting everything together, we arrive at the following result for the integral $I_{30}$

$$
\begin{aligned}
I_{30}= & \frac{3}{8 \epsilon^{4}}+\frac{13}{6 \epsilon^{3}}+\frac{1}{\epsilon^{2}}\left(-\frac{109}{12}-\frac{\pi^{2}}{12}\right)+\frac{1}{\epsilon}\left(-31 \zeta_{3}+\frac{5 \pi^{2}}{9}+\frac{461}{12}\right)-76 \zeta_{3} \\
& -\frac{1969}{12}-\frac{23 \pi^{2}}{18}-\frac{1211 \pi^{4}}{360}+\epsilon\left(-\frac{311}{6} \pi^{2} \zeta_{3}+292 \zeta_{3}-\frac{3111 \zeta_{5}}{2}+\frac{7 \pi^{2}}{18}+\frac{8501}{12}\right. \\
& \left.-\frac{355 \pi^{4}}{36}\right)+\epsilon^{2}\left(18 \zeta_{3}^{2}-\frac{727 \pi^{2} \zeta_{3}}{3}-1108 \zeta_{3}-4035 \zeta_{5}+\frac{1147 \pi^{4}}{30}\right. \\
& \left.+\frac{397 \pi^{2}}{18}-\frac{37129}{12}-\frac{10729 \pi^{6}}{810}\right) .
\end{aligned}
$$

Finally, using reduction to master integrals and explicit expressions for integrals given in the supplementary material, we arrive at the following result for the integral of $\omega_{n \bar{n}}^{(3), c}$ over the $n n n$ phase space

$$
\begin{aligned}
& \int \mathrm{d} \Phi_{\theta \theta \theta}^{n n n} \omega_{n \bar{n}}^{(3), c}\left(k_{1}, k_{2}, k_{3}\right) \\
= & -\frac{4}{\epsilon^{5}}+\frac{70}{3 \epsilon^{4}}+\frac{1}{\epsilon^{3}}\left(\frac{920}{9}+\frac{19 \pi^{2}}{3}\right)+\frac{1}{\epsilon^{2}}\left(\frac{8527}{27}+\frac{122 \pi^{2}}{3}+162 \zeta_{3}\right) \\
& +\frac{1}{\epsilon}\left(\frac{67193}{81}+\frac{1280 \pi^{2}}{9}+\frac{197 \pi^{4}}{90}+\frac{2732 \zeta_{3}}{3}\right)+\left(\frac{558745}{243}+\frac{10990 \pi^{2}}{27}+\frac{439 \pi^{4}}{9}\right. \\
& \left.+\frac{32032 \zeta_{3}}{9}-\frac{1604 \pi^{2} \zeta_{3}}{3}+4204 \zeta_{5}\right)+\epsilon\left(\frac{4074557}{729}+\frac{89138 \pi^{2}}{81}+\frac{28024 \pi^{4}}{135}\right. \\
& \left.-\frac{23029 \pi^{6}}{2835}+\frac{288992 \zeta_{3}}{27}-\frac{9224 \pi^{2} \zeta_{3}}{3}-7604 \zeta_{3}^{2}+50296 \zeta_{5}\right) .
\end{aligned}
$$




\subsection{Differential equations and $\omega_{n \bar{n}}^{(3), d}$ contribution}

Application of integration-by-parts identities to phase-space integrals with Heaviside functions opens up an opportunity to compute complicated integrals using differential equations. We will illustrate this by considering the contribution of the function $\omega_{n \bar{n}}^{(3), d}$, constructed using the function $\overline{\mathcal{S}}_{i k}^{(d)}$ defined in ref. [61], to the zero-jettiness soft function. The function $\overline{\mathcal{S}}_{i k}^{(d)}$ reads

$$
\begin{aligned}
& \overline{\mathcal{S}}_{i k}^{(d)}=\frac{-\left(p_{i} p_{k}\right)^{2}}{4 q_{123}^{2} p_{k} q_{123} p_{k} q_{1} p_{i} q_{2} p_{i} q_{3}}+\frac{1}{4 q_{123}^{2} p_{k} q_{123}}\left\{\frac { 1 } { q _ { 1 } q _ { 2 } } \left[\frac { 1 } { p _ { k } q _ { 1 } p _ { i } q _ { 2 } } \left(p_{i} p_{k}\left(q_{2} q_{3}-q_{1} q_{3}\right)\left(\frac{p_{i} p_{k}}{p_{i} q_{3}}\right)\right.\right.\right. \\
& \left.+2 \frac{p_{i} p_{k}}{p_{i} q_{3}}\left(p_{i} q_{12} p_{k} q_{123}-p_{i} q_{3} p_{k} q_{2}-p_{i} q_{2} p_{k} q_{3}\right)\right) \\
& +\frac{1}{2 p_{i} q_{13} p_{k} q_{2}}\left\{\left(\frac{1}{p_{i} q_{1}}-\frac{1}{p_{i} q_{3}}\right)\right. \\
& \times\left[p_{i} p_{k}\left(p_{k} q_{1} p_{i}\left(4 q_{3}-3 q_{12}\right)-3 p_{k} q_{2} p_{i} q_{12}+p_{k} q_{3} p_{i}\left(q_{1}-3 q_{2}\right)+2 p_{i} p_{k}\left(q_{2} q_{3}-q_{1} q_{3}\right)\right)\right] \\
& \left.+4 p_{i} p_{k} p_{k}\left(q_{3}-q_{1}\right)\right\} \\
& +\frac{1}{p_{i} q_{12} p_{k} q_{3}}\left\{\left(\frac{1}{p_{i} q_{1}}-\frac{1}{p_{i} q_{2}}\right)\left(-2 p_{i} p_{k} p_{i} q_{1} p_{k} q_{123}\right)\right. \\
& \left.+p_{i} p_{k} p_{k}\left(q_{1}-3 q_{2}-q_{3}\right)\right\}+\frac{1}{p_{k} q_{13} p_{i} q_{2}}\left\{p_{i} p_{k} p_{k} q_{13}\right. \\
& \left.+\left(\frac{1}{p_{k} q_{3}}-\frac{1}{p_{k} q_{1}}\right)\left[\frac{p_{i} p_{k}}{2} p_{k} q_{123} p_{k} q_{12}\right]\right\}+\frac{1}{p_{k} q_{12} p_{i} q_{3}}\left\{p_{i} p_{k} p_{k}\left(3 q_{1}+q_{2}-3 q_{3}\right)\right. \\
& \left.+\left(\frac{1}{p_{k} q_{2}}-\frac{1}{p_{k} q_{1}}\right)\left[2 p_{i} p_{k} p_{k} q_{1} p_{k} q_{13}\right]\right\} \\
& +\frac{2}{p_{i} q_{123}}\left\{\left(\frac{p_{k} q_{13}}{p_{k} q_{2}}-1\right)\left(\frac{p_{i} p_{k}}{2}\right)+\left(\frac{1}{p_{k} q_{12}}-\frac{1}{p_{k} q_{3}}\right)\left(-p_{i} p_{k} p_{k}\left(q_{2}+2 q_{3}\right)\right)\right. \\
& +\frac{1}{3}\left(\frac{1}{p_{k} q_{3}}-\frac{3}{p_{k} q_{12}}\right)\left(\frac{1}{p_{k} q_{1}}-\frac{1}{p_{k} q_{2}}\right)\left(2 p_{i} p_{k} p_{k} q_{2} p_{k}\left(q_{12}-q_{3}\right)\right) \\
& \left.\left.+\frac{1}{12}\left(\frac{1}{p_{k} q_{2}}-\frac{3}{p_{k} q_{13}}\right)\left(\frac{1}{p_{k} q_{1}}-\frac{1}{p_{k} q_{3}}\right)\left[4 p_{i} p_{k} p_{k}\left(q_{1}-q_{2}\right) p_{k}\left(q_{3}-q_{12}\right)\right]\right\}\right] \\
& +\frac{1}{\left(q_{1} q_{2}\right)^{2}}\left[\frac{2}{p_{i} q_{123}}\left(\frac{1}{p_{k} q_{3}}-\frac{1}{p_{k} q_{12}}\right)\right. \\
& \times\left[p_{i} p_{k} q_{1} q_{3}\left((d-4) p_{k} q_{1}-d p_{k} q_{2}\right)+2(d-2) p_{i} q_{2}\left(p_{k} q_{1}\right)^{2}+p_{k} q_{1} p_{k} q_{2}\left((4-d) p_{i} q_{1}+\frac{d}{2} p_{i} q_{3}\right)\right] \\
& +\frac{1}{p_{i} q_{12} p_{k} q_{3}}\left\{(d-2) p_{i}\left(q_{2}-q_{1}\right) p_{k} q_{1} p_{k} q_{13}\right\} \\
& \left.+\frac{1}{p_{k} q_{12} p_{i} q_{3}}\left\{2 p_{i} p_{k} q_{1} q_{3}\left((4-d) p_{k} q_{1}+d p_{k} q_{2}\right)+(d-2) p_{i} q_{1} p_{k}\left(q_{1}-q_{2}\right) p_{k}\left(q_{13}-3 q_{2}\right)\right\}\right] \\
& +\frac{1}{2 q_{1} q_{2} q_{1} q_{3}}\left[\frac { 1 } { p _ { i } q _ { 1 2 } p _ { k } q _ { 3 } } \left\{4 p_{i} p_{k} q_{2} q_{3} p_{k}\left(2 q_{1}+q_{3}\right)\right.\right. \\
& +\left(p_{k} q_{1}\right)^{2}\left((7-2 d) p_{i} q_{1}+(2 d+1) p_{i} q_{2}-4 p_{i} q_{3}\right)+2 p_{k} q_{1} p_{k} q_{3}\left((5-d) p_{i} q_{1}+(d-5) p_{i} q_{2}-2 p_{i} q_{3}\right) \\
& +p_{k} q_{1} p_{k} q_{2}\left((2 d-3) p_{i} q_{1}+(9-2 d) p_{i} q_{2}-2 p_{i} q_{3}\right)+3\left(p_{k} q_{3}\right)^{2} p_{i}\left(q_{1}-q_{2}\right)
\end{aligned}
$$




$$
\begin{aligned}
& \left.+p_{k} q_{2} p_{k} q_{3} p_{i}\left(9 q_{1}-3 q_{2}+2 q_{3}\right)+2\left(p_{k} q_{2}\right)^{2} p_{i}\left(q_{3}-q_{1}\right)\right\} \\
& +\frac{1}{p_{k} q_{12} p_{i} q_{3}}\left\{2 p_{i} p_{k} q_{2} q_{3} p_{k}\left(q_{12}+2 q_{3}\right)+\left(p_{k} q_{1}\right)^{2}\left((2 d-7) p_{i} q_{1}+2 p_{i} q_{2}+p_{i} q_{3}\right)\right. \\
& +p_{k} q_{1} p_{k} q_{3}\left((2 d-7) p_{i} q_{1}+4 p_{i} q_{2}-3 p_{i} q_{3}\right)+2 p_{k} q_{1} p_{k} q_{2}\left(p_{i} q_{2}-2 d p_{i} q_{1}\right)+2\left(p_{k} q_{3}\right)^{2} p_{i}\left(2 q_{1}-q_{2}\right) \\
& \left.+p_{k} q_{2} p_{k} q_{3}\left(2 p_{i} q_{2}+3 p_{i} q_{3}-(2 d+1) p_{i} q_{1}\right)+\left(p_{k} q_{2}\right)^{2}\left((2 d-9) p_{i} q_{1}-p_{i} q_{3}\right)\right\} \\
& +\frac{4}{p_{i} q_{123}}\left(\frac{1}{p_{k} q_{12}}-\frac{1}{p_{k} q_{3}}\right)\left\{q_{2} q_{3}\left[\frac{p_{i} p_{k}}{2} p_{k}\left(5 q_{1}-3 q_{2}+4 q_{3}\right)\right]+\left(p_{k} q_{2}\right)^{2} p_{i} q_{3}\right. \\
& +\left(p_{k} q_{1}\right)^{2}\left(p_{i} q_{1}+(3-d) p_{i} q_{2}\right) \\
& \left.\left.\left.+(d-2) p_{k} q_{1} p_{k} q_{2} p_{i} q_{2}+p_{k} q_{1} p_{k} q_{3} p_{i}\left(q_{1}-3 q_{2}\right)+p_{k} q_{2} p_{k} q_{3} p_{i}\left(3 q_{1}-q_{2}\right)\right\}\right]\right\} \\
& +\frac{1}{2\left(q_{123}^{2}\right)^{2} p_{i} q_{123} p_{k} q_{123}}\left\{(3 d-10) p_{i} p_{k}+\frac{2 p_{i} p_{k} q_{1} q_{3}}{\left(q_{1} q_{2}\right)^{2}}\left((d-4) q_{1} q_{3}-d q_{2} q_{3}\right)\right. \\
& +\frac{1}{q_{1} q_{2}}\left[p_{k} q_{1}\left((8-3 d) p_{i} q_{1}+(16-7 d) p_{i} q_{2}\right)-\frac{d}{2} p_{k} q_{3} p_{i} q_{3}-2 p_{i} p_{k}\left(2 q_{1} q_{3}+3 q_{2} q_{3}\right)\right] \\
& \left.+\frac{q_{2} q_{3}}{q_{1} q_{2} q_{1} q_{3}}\left[p_{i} p_{k} q_{2} q_{3}+4(d-4) p_{k} q_{1} p_{i} q_{1}-16 p_{k} q_{1} p_{i} q_{2}+4(2-d) p_{k} q_{2} p_{i} q_{2}\right]\right\}
\end{aligned}
$$

where $p_{i}=n, p_{k}=\bar{n}$ and $q_{i}=k_{i}, i=1,2,3$ in our notations. A distinct feature of the function $\overline{\mathcal{S}}_{i k}^{(d)}$ is that it contains a propagator $1 / k_{123}^{2}$ which depends on the relative orientation of all three gluons in the plane transverse to the collision axis. This feature makes it very difficult to integrate the function $\omega_{n \bar{n}}^{(3), d}$ analytically over the phase space with $\theta$-functions. On the other hand, the ability to write down the IBP equations and perform reduction to master integrals should allow us to use differential equations to compute even the most complicated master integrals. This is what we would like to discuss in this section.

We begin by expressing the integral of $\omega_{n \bar{n}}^{(3), d}$ through master integrals. These master integrals can be found in appendix A; they can be split into two groups, depending on whether they contain the $1 / k_{123}^{2}$ propagator or not. The master integrals from the first group are similar to what we have discussed earlier; they can be calculated in a similar manner and the results are provided in the supplementary material. Master integrals that belong to the second group involve a propagator $1 / k_{123}^{2}$. These are the most complicated integrals and we decided to compute them numerically.

To this end, we replace a propagator $1 / k_{123}^{2}$ with $1 /\left(k_{123}^{2}+m^{2}\right)$ in all master integrals that contribute to the integral of $\omega_{n \bar{n}}^{(3), d}$ and derive differential equations for these integrals w.r.t. the mass parameter $m^{2}$. We emphasize that the very possibility to use differential equations for phase-space integrals with Heaviside functions depends on our ability to set up integration-by-parts identities and reduce the derivatives of master integrals back to basis integrals.

It is quite clear that additional mass parameter makes integration-by-parts identities more complex and requires us to introduce more integrals to close them. However, once the differential equations are derived, it is in principle straightforward to compute the required integrals by solving them numerically. We do this by using the method described in ref. [65]. 
In what follows, we first discuss calculation of boundary conditions and then explain how to solve differential equations by considering a (relatively) simple example.

\subsubsection{Calculation of the boundary conditions}

We have seen in the previous sections that, although IBP reductions have to be performed for a non-vanishing value of the analytic regulator, the limit $\nu \rightarrow 0$ can be taken in an absolute majority of integrals after the reduction is performed. The same applies to integrals that contain propagators $1 / k_{123}^{2}$ that we modify by introducing the mass parameter $m$. We will therefore discuss calculation of boundary conditions for such integrals, setting the analytic regulator to zero. ${ }^{12}$

The complexity of the boundary conditions computation strongly depends on the type of constraints that a particular integral is subject to. As we explain below, the more $\delta$-functions a particular integral has, the easier it is to compute the boundary conditions. To understand this, consider integrals with three $\delta$-functions

$$
I_{\delta \delta \delta}(m)=\int \frac{\mathrm{d} \Phi_{\delta \delta \delta}^{n n n}}{\left(k_{123}^{2}+m^{2}\right)^{i} \ldots} .
$$

In eq. (5.50) the ellipses stand for mass-parameter-independent scalar products and $i$ is an integer number. In all integrals that refer to the $n n n$ configuration, all $\beta$-variables are restricted because $\beta_{i}>0, i=1,2,3$ and $\beta_{1}+\beta_{2}+\beta_{3}=1$. Moreover, for integrals with three $\delta$-functions, all $\alpha$-variables are equal to $\beta$-variables and $k_{i, \perp}^{2}=\alpha_{i} \beta_{i}=\beta_{i}^{2}, i=1,2,3$. Hence, integration in eq. (5.50) is performed over a finite region of the three-particle phase space, so that the approximate form of the integral in the $m^{2} \rightarrow \infty$ limit is simply obtained by expanding the propagator $1 /\left(k_{123}^{2}+m^{2}\right)$ in $k_{123}^{2} / m^{2}$. It follows that all $I_{\delta \delta \delta}$ integrals have a particularly simple asymptotic mass dependence

$$
\lim _{m \rightarrow \infty} I_{\delta \delta \delta}(m) \sim m^{-2 i} .
$$

It is obvious that a Taylor expansion of $I_{\delta \delta \delta}$-integrals in $k_{123}^{2} / \mathrm{m}^{2}$ produces integrals where a "massive" propagator is absent. As the result, once the expansion is performed, we can use integration by parts for $k_{123}^{2}$-independent integrals to express any expanded $I_{\delta \delta \delta}$ integral through master integrals computed in the previous sections.

Consider now an integral that contains two $\delta$-functions and one $\theta$-function. We choose four-momenta $k_{1,2,3}$ in such a way that the $\theta$-function depends on $k_{1}$ (more precisely on $\alpha_{1}$ and $\beta_{1}$ ) and write

$$
I_{\theta \delta \delta}(m)=\int \frac{\mathrm{d} \Phi_{\theta \delta \delta}^{n n n}}{\left(k_{123}^{2}+m^{2}\right)^{i} \ldots} .
$$

At variance with $I_{\delta \delta \delta}$ integrals, the integration over $\alpha_{1}$ in $I_{\theta \delta \delta}$ integrals is not restricted from above. This implies that, in the limit $m \rightarrow \infty$, there are contributions from the region $\alpha_{1} \sim m$ as well as $\alpha_{1} \sim 1$. Since the phase-space element scales as $\alpha_{1}^{-\epsilon}$ and since in the

\footnotetext{
${ }^{12}$ There are two integrals $J_{\nu}$ and $J_{\nu}^{(d)}$ (defined in eq. (5.18) and eq. (A.6)) that exhibit $1 / \nu$ behavior and enter $\omega_{n \bar{n}}^{(3), d}$ through the combination $\nu J_{\nu}$ and $\nu J_{\nu}^{(d)}$. The differential equation relevant to $\nu J_{\nu}$ and $\nu J_{\nu}^{(d)}$ is regular in $\nu$, which can be solved in exactly the same way as described in the following.
} 
limit $\alpha_{1} \rightarrow \infty, k_{123}^{2}+m^{2} \sim \alpha_{1}\left(\beta_{2}+\beta_{3}\right) \cdots+m^{2}$, we find that all integrals $I_{\theta \delta \delta}$ have the following asymptotic dependence on the mass parameter $m$ in the $m \rightarrow \infty$ limit

$$
\lim _{m \rightarrow \infty} I_{\theta \delta \delta}(m) \sim m^{-2 i_{1}-2 \epsilon} A_{2}+m^{-2 i} A_{1} .
$$

In this formula $i_{1}, i$ are two integers that are particular to the integral under consideration, and $A_{1,2}$ are independent of the mass. To compute $A_{1}$ we need to Taylor-expand the integrand in $k_{123}^{2} / \mathrm{m}^{2}$ and then use integration by parts to reduce the resulting integrals to massless $\theta \delta \delta$-integrals. To compute $A_{2}$, we need to drop the $\theta\left(\alpha_{1}-\beta_{1}\right)$ constraint, as it is only relevant for $\alpha_{1} \sim \beta_{1} \sim 1$, and then simplify a particular integral under the assumption that $\alpha_{1} \sim m^{2} \gg \alpha_{2}, \alpha_{3}, \beta_{1}, \beta_{2}, \beta_{3}$. We note that in this case all the relevant integrals can be straightforwardly computed in a closed form in terms of hypergeometric functions.

Finally, we require integrals with two $\theta$-functions and one $\delta$-function ${ }^{13}$

$$
I_{\theta \theta \delta}=\int \frac{\mathrm{d} \Phi_{\theta \theta \delta}^{n n n}}{\left(k_{123}^{2}+m^{2}\right)^{i} \ldots} .
$$

The asymptotic $m \rightarrow \infty$ mass-dependence of such integrals reads

$$
\lim _{m \rightarrow \infty} I_{\theta \theta \delta}(m) \sim m^{-2 i_{2}-4 \epsilon} A_{3}+m^{-2 i_{1}-2 \epsilon} A_{2}+m^{-2 i} A_{1} .
$$

In eq. (5.55) $A_{3}$ receives contributions from the integration region with $\alpha_{1} \sim \alpha_{2} \sim m^{2}$, $A_{2}$ - from regions where either $\alpha_{1} \sim m^{2}, \alpha_{2} \sim 1$ or $\alpha_{2} \sim m^{2}, \alpha_{1} \sim 1$ and $A_{1}$ - from the region where $\alpha_{1} \sim \alpha_{2} \sim 1$. We obtain $A_{1}$ upon Taylor expansion of an integrand in $k_{123}^{2} / m^{2}$ and $A_{2}$ upon Taylor expanding in "small" $\alpha$ - and $\beta$-parameters and neglecting the corresponding $\theta$-function constraint.

To illustrate how contributions of different regions can be computed, we consider one of the integrals with $1 /\left(k_{123}^{2}+m^{2}\right)$ propagator

$$
B_{1, \delta \theta \theta}=\int \frac{\mathrm{d} \Phi_{\delta \theta \theta}^{n n n}}{\left(k_{123}^{2}+m^{2}\right)\left(k_{1} k_{3}\right)\left(k_{12} n\right)\left(k_{3} \bar{n}\right)} .
$$

We would like to compute the leading contribution to $\mathcal{O}\left(m^{-2 \epsilon}\right)$ branch of this integrals in the limit $m \rightarrow \infty$. We consider two distinct contributions $\alpha_{2} \sim m^{2} \gg 1$ and $\alpha_{3} \sim m^{2} \gg 1$. In the first case, $\left[\mathrm{d} k_{2}\right] \sim \mathrm{d} \alpha_{2} \alpha_{2}^{-\epsilon}, k_{123}^{2}+m^{2} \sim \alpha_{2}\left(\beta_{1}+\beta_{3}\right) \cdots+m^{2}, k_{1} k_{3} \sim 1, k_{12} n \sim \beta_{12} \sim$ $1, k_{3} \bar{n} \sim \alpha_{3} \sim 1$. Hence, we conclude that

$$
\left.\lim _{m \rightarrow \infty} B_{1, \delta \theta \theta}\right|_{\alpha_{2} \sim m^{2}} \sim \int \frac{\mathrm{d} \alpha_{2} \alpha_{2}^{-\epsilon}}{\alpha_{2}\left(\beta_{1}+\beta_{3}\right)+m^{2}} \sim m^{-2 \epsilon} .
$$

However, in the case $\alpha_{3} \sim m^{2}$, there are additional dependences of the integrand on $k_{3}$ including $k_{3} k_{1} \sim \alpha_{3} \beta_{1} \sim m^{2}$ and $k_{3} \bar{n} \sim \alpha_{3} \sim m^{2}$. This implies that

$$
\left.\lim _{m \rightarrow \infty} B_{1, \delta \theta \theta}\right|_{\alpha_{3} \sim m^{2}} \sim \int \frac{\mathrm{d} \alpha_{3} \alpha_{3}^{-\epsilon}}{\left(\alpha_{3}\left(\beta_{1}+\beta_{2}\right)+m^{2}\right) \alpha_{3} \alpha_{3}} \sim m^{-2 \epsilon-4} .
$$

\footnotetext{
${ }^{13}$ As we explained earlier, there are no master integrals with three theta-functions in case of the $n n n$ configuration.
} 
We conclude that the leading $m \rightarrow \infty$ contribution to the $\mathcal{O}\left(m^{-2 \epsilon}\right)$ branch of the integral $B_{1, \delta \theta \theta}$ in eq. (5.56) arises from the region where $\alpha_{2} \sim m^{2}$ and all other variables are of order one.

To compute this contribution, we notice that, upon taking the limit $\alpha_{2} \sim m^{2} \rightarrow \infty$ in $k_{123}^{2}+m^{2}$, the only dependence of the integrand on relative azimuthal angles of massless partons resides in the simple scalar products, e.g. $k_{1} k_{3}$ in $B_{1}$. This integration can be easily performed following the discussion in the previous sections. We find

$$
\begin{aligned}
\left.B_{1, \delta \theta \theta}\right|_{\alpha_{2} \sim m^{2}}= & 2 \int \frac{\mathrm{d} \beta_{1} \mathrm{~d} \beta_{2} \mathrm{~d} \beta_{3} \beta_{1}^{-2 \epsilon} \beta_{2}^{-\epsilon} \beta_{3}^{-\epsilon} \delta\left(1-\beta_{123}\right) \mathrm{d} \alpha_{3} \alpha_{3}^{-\epsilon} \theta\left(\alpha_{3}-\beta_{3}\right)}{\left(\beta_{1}+\beta_{2}\right) \beta_{1} \alpha_{3}^{2}} \\
& \times{ }_{2} F_{1}\left[\{1,1+\epsilon\},\{1-\epsilon\} ; \frac{\beta_{3}}{\alpha_{3}}\right] \int_{0}^{\infty} \frac{\mathrm{d} \alpha_{2} \alpha_{2}^{-\epsilon}}{\alpha_{2} \beta_{13}+m^{2}} .
\end{aligned}
$$

We integrate over $\alpha_{2}$ and obtain

$$
\int_{0}^{\infty} \frac{\mathrm{d} \alpha_{2} \alpha_{2}^{-\epsilon}}{\alpha_{2} \beta_{13}+m^{2}}=m^{-2 \epsilon}\left(\beta_{13}\right)^{\epsilon-1} \Gamma(\epsilon) \Gamma(1-\epsilon) .
$$

We then change variables $\alpha_{3} \rightarrow \xi, \alpha_{3}=\beta_{3} / \xi$, observe that integration over $\xi$ factorizes and find

$$
\begin{aligned}
\left.B_{1, \delta \theta \theta}\right|_{\alpha_{2} \sim m^{2}}= & 2 m^{-2 \epsilon} \frac{\Gamma(1-\epsilon) \Gamma^{2}(1+\epsilon)}{\epsilon \Gamma(2+\epsilon)}{ }_{3} F_{2}[\{1,1+\epsilon, 1+\epsilon\},\{1-\epsilon, 2+\epsilon\} ; 1] \\
& \times \int \mathrm{d} \beta_{1} \mathrm{~d} \beta_{2} \mathrm{~d} \beta_{3} \delta\left(1-\beta_{123}\right) \beta_{1}^{-2 \epsilon-1} \beta_{2}^{-\epsilon} \beta_{3}^{-\epsilon} \beta_{12}^{-1} \beta_{13}^{\epsilon-1} \beta_{3}^{-\epsilon-1} .
\end{aligned}
$$

To compute the remaining integral, we remove the $\delta$-function by integrating over $\beta_{2}$ and change variables as follows $\beta_{1}=x y$ and $\beta_{3}=x(1-y)$. The integral over $x$ and $y$ is then of a hypergeometric type and we obtain the result for the required branch

$$
\begin{aligned}
& \left.B_{1, \delta \theta \theta}\right|_{\alpha_{2} \sim m^{2}}=2 m^{-2 \epsilon} \frac{\Gamma^{2}(1-\epsilon) \Gamma^{2}(1+\epsilon) \Gamma(-1-3 \epsilon) \Gamma^{2}(-2 \epsilon)}{\epsilon \Gamma(2+\epsilon) \Gamma^{2}(-4 \epsilon)} \\
& \times{ }_{3} F_{2}[\{1,1+\epsilon, 1+\epsilon\},\{1-\epsilon, 2+\epsilon\} ; 1]_{3} F_{2}[\{1,-1-3 \epsilon,-2 \epsilon\},\{-4 \epsilon,-4 \epsilon\} ; 1] .
\end{aligned}
$$

The computation of the asymptotic behavior of the $\mathcal{O}\left(m^{-2 \epsilon}\right)$ branch that we just described is typical. In fact, all such boundary conditions can be calculated in terms of hypergeometric functions in a straightforward manner.

It turns out that the most difficult boundary contributions to compute are the $\mathcal{O}\left(m^{-4 \epsilon}\right)$ branches of $I_{\theta \theta \delta}$ integrals. Indeed, for such branches, we need to consider asymptotic limits $\alpha_{1} \sim \alpha_{2} \sim m^{2} \gg \alpha_{3}, \beta_{1}, \beta_{2}, \beta_{3}$. Inspecting $k_{123}^{2}$ in this limit, we find that we cannot simplify the scalar product $k_{1} k_{2}$ so that $k_{123}^{2}+m^{2}$ still depends on the relative orientation of $k_{1}$ and $k_{2}$ in the transverse plane, even if $m$ is taken to infinity.

To understand how such integrals can be calculated, we note that for the computation of the $\mathcal{O}\left(m^{-4 \epsilon}\right)$ branch, we need to neglect all $\theta$-functions in the definition of $\theta \theta \delta$ integrals 
since they are only relevant for $\alpha_{1,2} \sim \beta_{1,2} \sim 1$ which violates the assumption $\alpha_{1,2} \sim m^{2}$. Hence, a $\mathcal{O}\left(m^{-4 \epsilon}\right)$ branch of any $\theta \theta \delta$ integral can be computed by writing

$$
\begin{aligned}
m^{-2 i_{1}-4 \epsilon} A_{3} \sim & \int \mathrm{d}^{d} q\left[\mathrm{~d} k_{3}\right] \frac{\delta\left(1-q n-k_{3} n\right) \delta\left(k_{3} \bar{n}-k_{3} n\right)}{\left(\left(k_{3}+q\right)^{2}+m^{2}\right)^{i}} \\
& \times \int\left[\mathrm{d} k_{1}\right]\left[\mathrm{d} k_{2}\right] \delta\left(q-k_{1}-k_{2}\right) \ldots,
\end{aligned}
$$

where ellipses stand for various scalar products of momenta that appear in a particular integral. All these scalar products have to be simplified under the assumption that $k_{1} \bar{n} \sim$ $k_{2} \bar{n} \sim m^{2} \gg k_{1} n, k_{2} n, k_{3} n, k_{3} \bar{n}$. We can further write

$$
k_{123}^{2}=\left(q+k_{3}\right)^{2}=q^{2}+2 k_{3} q \rightarrow q^{2}+\beta_{3}(q \bar{n})=q^{2}+(1-q n)(q \bar{n}) .
$$

Therefore, to determine the $\mathcal{O}\left(m^{-4 \epsilon}\right)$ branch of any $I_{\theta \theta \delta}$ integral, we need to compute

$$
\begin{aligned}
m^{-2 i_{1}-4 \epsilon} A_{3} & \sim \int \frac{\mathrm{d}^{d} q}{\left(q^{2}+(1-q n)(q \bar{n})+m^{2}\right)^{i}} F\left(q^{2}, q n, q \bar{n}\right), \\
F\left(q^{2}, q n, q \bar{n}\right) & =\int\left[\mathrm{d} k_{3}\right] \delta\left(1-q n-k_{3} n\right) \delta\left(k_{3} \bar{n}-k_{3} n\right) \int\left[\mathrm{d} k_{1}\right]\left[\mathrm{d} k_{2}\right] \delta\left(q-k_{1}-k_{2}\right) \ldots
\end{aligned}
$$

At this point, we can again use the integration-by-parts method and express all relevant integrals $F\left(q^{2}, q n, q \bar{n}\right)$ as linear combinations of five master integrals. They read

$$
\begin{aligned}
& \left\{\mathcal{B}_{1}, \mathcal{B}_{2}, \mathcal{B}_{3}, \mathcal{B}_{4}, \mathcal{B}_{5}\right\} \\
& =\int\left[\mathrm{d} k_{3}\right] \delta\left(1-q n-k_{3} n\right) \delta\left(k_{3} \bar{n}-k_{3} n\right) \int\left[\mathrm{d} k_{1}\right]\left[\mathrm{d} k_{2}\right] \delta\left(q-k_{1}-k_{2}\right) \\
& \quad \times\left\{1, \frac{1}{k_{2} \bar{n}}, \frac{1}{\left(k_{2} n\right)\left(k_{2} \bar{n}\right)}, \frac{1}{\left(k_{2} n\right)\left(k_{1} \bar{n}\right)}, \frac{1}{1-k_{1} n}\right\} .
\end{aligned}
$$

Note that these integrals do not contain scalar products of the gluon four-momenta. This happens because such scalar products are either simple as e.g. in case of $k_{1} k_{2}=q^{2} / 2$ or can be simplified for large $\alpha_{1,2}$ as e.g. in $k_{3} k_{1} \rightarrow\left(k_{3} n\right)\left(k_{1} \bar{n}\right), k_{3} k_{2} \rightarrow\left(k_{3} n\right)\left(k_{2} \bar{n}\right)$.

It is convenient to compute the integrals $\mathcal{B}_{1, . .5}$ in the rest frame of $q$ where $k_{1,2}$ are back-to-back. Then, using the following result for the angular integral

$$
\int \frac{\mathrm{d} \Omega_{k}^{(d-1)}}{\left(1-\vec{n}_{k} \vec{n}\right)\left(1-\vec{n}_{k} \underline{\vec{r}}\right)}=2^{-2 \epsilon} \Omega^{(d-2)} \frac{\Gamma(1-\epsilon) \Gamma(-\epsilon)}{\Gamma(1-2 \epsilon)}{ }_{2} F_{1}\left[\{1,1\},\{1-\epsilon\} ; \frac{1+\vec{n} \underline{\vec{n}}}{2}\right],
$$

we easily find

$$
\begin{aligned}
& \mathcal{B}_{1}=\tilde{N}_{\epsilon}\left(q^{2}\right)^{-\epsilon}(1-q n)^{-2 \epsilon}, \\
& \mathcal{B}_{2}=\tilde{N}_{\epsilon} \frac{(1-2 \epsilon)}{(-\epsilon)} \frac{\left(q^{2}\right)^{-\epsilon}(1-q n)^{-2 \epsilon}}{q \bar{n}}, \\
& \mathcal{B}_{3}=\tilde{N}_{\epsilon} \frac{(1-2 \epsilon)}{(-\epsilon)} \frac{2\left(q^{2}\right)^{-\epsilon}(1-q n)^{-2 \epsilon}}{(q n)(q \bar{n})^{2}}{ }_{2} F_{1}\left[\{1,1\},\{1-\epsilon\} ; 1-\frac{q^{2}}{(q n)(q \bar{n})}\right], \\
& \mathcal{B}_{4}=\tilde{N}_{\epsilon} \frac{(1-2 \epsilon)}{(-\epsilon)} \frac{2\left(q^{2}\right)^{-\epsilon}(1-q n)^{-2 \epsilon}}{(q n)(q \bar{n})^{2}}{ }_{2} F_{1}\left[\{1,1\},\{1-\epsilon\} ; \frac{q^{2}}{(q n)(q \bar{n})}\right], \\
& \mathcal{B}_{5}=\tilde{N}_{\epsilon} q^{-2 \epsilon}(1-q n)^{-2 \epsilon}{ }_{2} F_{1}[\{1,1-\epsilon\},\{2-2 \epsilon\} ; q n],
\end{aligned}
$$


where

$$
\tilde{N}_{\epsilon}=\frac{1}{(2 \pi)^{d-1}}\left[\frac{\Omega^{(d-2)}}{4(2 \pi)^{d-1}}\right]^{2} \frac{\Gamma^{2}(1-\epsilon)}{\Gamma(2-2 \epsilon)} .
$$

A typical function $F\left(q^{2}, q n, q \bar{n}\right)$ is given by a linear combination of the integrals $\mathcal{B}_{1, . ., 5}$. For example, for one of the boundary integrals that we will refer to as $B_{2}$ this function reads

$$
\begin{aligned}
F_{2}\left(q^{2}, q n, q \bar{n}\right)= & {\left[\frac{4(1-2 \epsilon)^{2}(2-q n)(1-q n)}{(1+\epsilon) m^{6}(q n)^{2}} \mathcal{B}_{1}+\frac{4 \epsilon^{2}}{(1+\epsilon) m^{4}} \mathcal{B}_{2}\right.} \\
& \left.-\frac{2(1+2 \epsilon)(1-q n) q \bar{n}}{m^{2} q^{2}} \mathcal{B}_{4}\right] .
\end{aligned}
$$

Hence, to determine the $m^{-4 \epsilon}$ branch of the corresponding integral, we need to compute

$$
B_{2}=\int \frac{\mathrm{d}^{d} q \theta(1-q n)}{q^{2}+q \bar{n}(1-q n)+m^{2}} F_{2}\left(q^{2}, q n, q \bar{n}\right) .
$$

To calculate the integral in eq. (5.71), we need to choose a convenient parameterization to integrate over $q$. We do this in the following way. We introduce the Sudakov decomposition for the vector $q$ and write $q=\frac{1}{2} \alpha_{q} n+\frac{1}{2} \beta_{q} \bar{n}+q_{\perp}$. It follows that

$$
\mathrm{d}^{d} q \theta(1-q n)=\frac{1}{4} \mathrm{~d} \alpha_{q} \mathrm{~d} \beta_{q} \mathrm{~d} q_{\perp}^{2}\left(q_{\perp}^{2}\right)^{-\epsilon} \mathrm{d} \Omega^{(d-2)} \theta\left(1-\beta_{q}\right) .
$$

In addition, the four-vector $q$ needs to be time-like, $q^{2}>0$. This implies

$$
q^{2}=\alpha_{q} \beta_{q}-q_{\perp}^{2}>0
$$

We would like to simplify the mass-dependent denominator in eq. (5.71). To do that, we first re-write it using Sudakov variables

$$
q^{2}+q \bar{n}(1-q n)+m^{2}=\alpha_{q}-q_{\perp}^{2}+m^{2}=t+m^{2},
$$

where we introduce a new variable $t=\alpha_{q}-q_{\perp}^{2}$. Positivity of $q^{2}$ requires

$$
\alpha_{q}>\frac{q_{\perp}^{2}}{\beta_{q}}
$$

This implies

$$
\begin{aligned}
& t=\alpha_{q}-q_{\perp}^{2}>\frac{q_{\perp}^{2}\left(1-\beta_{q}\right)}{\beta_{q}}>0 \\
& q^{2}>0 \Rightarrow t-\alpha_{q}\left(1-\beta_{a}\right)>0 .
\end{aligned}
$$

Hence, we can write

$$
\mathrm{d}^{d} q \theta(1-q n)=\frac{1}{4} \mathrm{~d} \alpha_{q} \mathrm{~d} \beta_{q} \mathrm{~d} t\left(\alpha_{q}-t\right)^{-\epsilon} \mathrm{d} \Omega^{(d-2)} \theta\left(1-\beta_{q}\right),
$$


where the integration boundaries are $0<\beta_{q}<1,0<\alpha_{q}<\infty$ and $\alpha_{q}\left(1-\beta_{q}\right)<t<\alpha_{q}$. Using the fact that the integrand in eq. (5.71) does not depend on the azimuthal angle, we integrate over $\mathrm{d} \Omega^{(d-2)}$ and write

$$
\begin{aligned}
B_{2}= & \frac{\Omega^{(d-2)}}{4} \int \frac{\mathrm{d} \alpha_{q} \mathrm{~d} \beta_{q} \mathrm{~d} t\left(\alpha_{q}-t\right)^{-\epsilon}}{t+m^{2}} \theta\left(t-\alpha_{q}\left(1-\beta_{q}\right)\right) \\
& \times\left[\frac{4(1-2 \epsilon)^{2}\left(2-\beta_{q}\right)\left(1-\beta_{q}\right)}{(1+\epsilon) m^{6} \beta_{q}^{2}} \mathcal{B}_{1}+\frac{4 \epsilon^{2}}{(1+\epsilon) m^{4}} \mathcal{B}_{2}-\frac{2(1+2 \epsilon)\left(1-\beta_{q}\right) \alpha_{q}}{m^{2}\left(t-\alpha_{q}\left(1-\beta_{q}\right)\right)} \mathcal{B}_{4}\right] .
\end{aligned}
$$

To integrate further, we change variables $\alpha_{q} \rightarrow \xi$ with $\alpha_{q}=t / \xi$ and $0<\xi<1$. Since

$$
q^{2}=t-\alpha_{q}\left(1-\beta_{q}\right)=\frac{t}{\xi}\left(\xi-\left(1-\beta_{q}\right)\right)>0,
$$

the integration boundary for $\beta_{q}$ becomes $1-\xi<\beta_{q}<1$. To accommodate these boundaries in a natural way, we change variables $\beta \rightarrow r$, with $\beta=1-\xi r, 0<r<1$.

Upon changing variables and using explicit expressions for integrals $\mathcal{B}_{1,2,4}$, we note that integration over $t$ factorizes and can be performed easily. We obtain

$$
\begin{aligned}
& B_{2}=-\frac{m^{-4-4 \epsilon} \Gamma^{2}(1-\epsilon) \Gamma(1+2 \epsilon)}{\epsilon^{2}} \int_{0}^{1} \mathrm{~d} \xi \mathrm{d} r W_{2}(\xi, r), \\
& W_{2}(\xi, r)=\left\{2 \epsilon \frac{\left(r(1+r \xi)+\epsilon\left(1-2 r(1+\xi)-2 r^{2} \xi(1-\xi / 2)\right)\right)}{(1+\epsilon)(1-r)^{\epsilon} r^{2 \epsilon}(1-\xi)^{\epsilon} \xi^{\epsilon}(1-r \xi)^{2}}\right. \\
& \\
&\left.+2(2 \epsilon+1) \frac{r^{1-2 \epsilon} \xi^{1-\epsilon}(1-r \xi)^{\epsilon}}{(1-r)^{1+\epsilon}(1-\xi)^{1+2 \epsilon}}{ }_{2} F_{1}\left[\{-\epsilon,-\epsilon\},\{1-\epsilon\} ; \frac{(1-r) \xi}{1-r \xi}\right]\right\} .
\end{aligned}
$$

Integrating over $\xi$ and $r$, we find

$$
\begin{aligned}
B_{2}= & m^{-4 \epsilon-4}\left(-\frac{3}{2 \epsilon^{4}}-\frac{6}{\epsilon^{3}}-\frac{12}{\epsilon^{2}}+\frac{15 \zeta_{3}-36}{\epsilon}+60 \zeta_{3}+\frac{\pi^{4}}{4}-84+\epsilon\left(120 \zeta_{3}\right.\right. \\
& \left.\left.+81 \zeta_{5}+\pi^{4}-204\right)+\epsilon^{2}\left(-75 \zeta_{3}^{2}+360 \zeta_{3}+324 \zeta_{5}+\frac{11 \pi^{6}}{63}+2 \pi^{4}-468\right)\right) .
\end{aligned}
$$

Calculation of $\mathcal{O}\left(m^{-4 \epsilon}\right)$ branches for other integrals that are needed to determine boundary conditions proceeds along similar lines. Integration over $t$ can always be performed exactly and the subsequent integration over $\xi$ and $r$ is then completed by constructing subtraction terms of end-point singularities to facilitate expansion of integrands in $\epsilon$.

\subsubsection{Numerical solution of the differential equations}

Having discussed the computation of the boundary conditions, we need to explicitly write down and solve the differential equations. We note that we need to consider about two hundred mass-dependent master integrals in total to close the system of differential equations. Since it is impossible to discuss such a huge system of differential equations in any detail, we decided to choose a small eleven-by-eleven sub-system and discuss it in a comprehensive way. 
We consider eleven integrals

$$
\begin{aligned}
\mathcal{J}_{1} & =\int \mathrm{d} \Phi_{\delta \delta \delta}^{n n n}, & \mathcal{J}_{2} & =\int \frac{\mathrm{d} \Phi_{\delta \delta \delta}^{n n n}}{k_{123}^{2}+m^{2}}, \\
\mathcal{J}_{3} & =\int \frac{\mathrm{d} \Phi_{\delta \delta \delta}^{n n n}}{\left(k_{123}^{2}+m^{2}\right) k_{1} \cdot n}, & \mathcal{J}_{4} & =\int \frac{\mathrm{d} \Phi_{\delta \delta \theta}^{n n n}}{k_{123}^{2}+m^{2}}, \\
\mathcal{J}_{5} & =\int \frac{\mathrm{d} \Phi_{\delta \delta \theta}^{n n n}}{\left(k_{123}^{2}+m\right)^{2}}, & \mathcal{J}_{6} & =\int \frac{\mathrm{d} \Phi_{\delta \delta^{\prime} \theta}^{n n}}{k_{123}^{2}+m^{2}}, \\
\mathcal{J}_{7} & =\int \frac{\mathrm{d} \Phi_{\delta \delta \theta}^{n n n}}{\left(k_{123}^{2}+m^{2}\right)\left(k_{1} n\right)}, & \mathcal{J}_{8} & =\int \frac{\mathrm{d} \Phi_{\delta \delta \theta}^{n n n}}{\left(k_{123}^{2}+m^{2}\right)\left(k_{3} \bar{n}\right)}, \\
\mathcal{J}_{9} & =\int \frac{\mathrm{d} \Phi_{\delta \theta \theta}^{n n n}}{k_{123}^{2}+m^{2}}, & \mathcal{J}_{10} & =\int \frac{\mathrm{d} \Phi_{\delta \theta \theta}^{n n n}}{\left(k_{123}^{2}+m^{2}\right)\left(k_{2} \bar{n}\right)}, \\
\mathcal{J}_{11} & =\int \frac{\mathrm{d} \tilde{\Phi}_{\delta \theta \theta}^{n n n}}{\left(k_{123}^{2}+m^{2}\right)\left(k_{2} \bar{n}\right)} . & &
\end{aligned}
$$

We note that somewhat different notations for the phase-space measures in $\mathcal{J}_{6}$ and $\mathcal{J}_{11}$, compared to other integrals, imply that derivatives of $\delta$-functions, namely $\mathrm{d} \delta\left(k_{2} \bar{n}-k_{2} n\right) / \mathrm{d}\left(k_{2} n\right)$ and $\mathrm{d} \delta\left(1-k_{123} n\right) / \mathrm{d} k_{123} n$ appear in $\mathcal{J}_{6}$ and $\mathcal{J}_{11}$ respectively. It is straightforward to compute these derivatives if the corresponding $\delta$-functions are represented using eq. (2.10) in the spirit of reverse unitarity.

To derive differential equations, we consider a vector of eleven integrals introduced in eq. (5.82), differentiate it with respect to $\mathrm{m}^{2}$, use integration-by-parts identities to perform a reduction of the resulting integrals back to the integrals $\mathcal{J}_{1, \ldots, 11}$ and obtain

$$
\frac{\partial}{\partial m^{2}} \mathcal{J}=\left[\frac{\boldsymbol{M}_{1}}{m^{2}+1}+\frac{\boldsymbol{M}_{2}}{m^{2}+\frac{1}{4}}+\frac{\boldsymbol{M}_{3}}{m^{2}}+\boldsymbol{M}_{4}+m^{2} \boldsymbol{M}_{5}\right] \mathcal{J} .
$$

The matrices $\boldsymbol{M}_{1, . .5}$ are independent of $m^{2}$ but depend on $\epsilon$. They can be found in appendix B.

We have already explained how to compute the boundary conditions in the previous section; explicit results for integrals $\mathcal{J}_{1, . ., 11}$ that appear in eq. (5.82) can be found in the supplementary material. With the boundary conditions at hand, we solve the differential equation in the following way. At $m^{2} \rightarrow i \infty$, all master integrals are written as powerlogarithmic series in $y=1 / m^{2}$

$$
\mathcal{J}=\sum \boldsymbol{C}_{\infty}(k, n) y^{k} \ln ^{n} y
$$

Coefficients of these series solutions are fixed with the help of boundary conditions and the differential equations. We can use these series solutions to evaluate integrals in the upper complex half-plane within its radius of convergence. The radius of convergence follows from eq. (5.83) where we observe singularities at $m^{2}=0,-1 / 4,-1$.

Suppose we take the point $m^{2}=m_{0}^{2}=1 / y_{0}$ where the solution eq. (5.84) is valid. We construct another series solution at this point

$$
\mathcal{J}=\sum \boldsymbol{C}_{\boldsymbol{y}_{\mathbf{0}}}(k)\left(y-y_{0}\right)^{k}
$$


and find coefficients $\boldsymbol{C}_{y_{0}}(k)$ by matching the above equation to eq. (5.84) at around $y=y_{0}$. The solution in eq. (5.85) has its own radius of convergence and allows us to move past the radius of convergence of the original solution eq. (5.84). We then repeat this procedure at another point $m^{2}=m_{1}^{2}$ closer to the physical point at $m^{2}=0$ and iterate.

After sufficient number of steps, we arrive at a point $m^{2}=m_{f}^{2}$ within the radius of convergence of the formal solution at $m^{2}=0$. As follows from the differential equation eq. (5.83) $\mathrm{m}^{2}=0$ is a regular singular point of the differential equation; to determine coefficients of the solution constructed as an expansion around $m^{2}=0$ we proceed as follows.

The formal solution in the neighborhood of the point $m^{2}=0$ can be written as

$$
\mathcal{J}\left(m^{2}\right)=\boldsymbol{P}\left(\varepsilon, m^{2}\right)\left(m^{2}\right)^{M(\varepsilon)} \mathcal{J}_{0}(\varepsilon)
$$

where $\boldsymbol{P}$ is a matrix that can be computed as a series in $m^{2}$ and the matrix $\boldsymbol{M}$ is related to $\boldsymbol{M}_{3}$ defined in eq. (5.83).

The matrix $\boldsymbol{P}$ can be found by constructing a series solution around $m^{2}=0$. The vector $\mathcal{J}_{0}$ corresponds to the boundary conditions at $m=0$; we can determine it by matching the formal solution against the evaluation at a finite- $m$ point $m_{f}^{2}$. We write

$$
\mathcal{J}_{0}(\varepsilon)=\left[\boldsymbol{P}\left(\varepsilon, m_{f}^{2}\right)\left(m_{f}^{2}\right)^{\boldsymbol{M}(\varepsilon)}\right]^{-1} \mathcal{J}\left(m_{f}^{2}\right)
$$

The above procedure allows us to obtain the complete solution $\mathcal{J}$ in the neighborhood of the point $m^{2}=0$, and the only thing left to do is to select integrals we are interested in.

By construction, these integrals correspond to a situation when the limit $m^{2} \rightarrow 0$ is taken while keeping $\epsilon$ fixed; this means that all contributions to integrals $\boldsymbol{J}$ that scale as $\left(m^{2}\right)^{i \epsilon+i_{1}} \ln ^{k} m^{2}$, where $i, i_{1}$ and $k$ are non-vanishing rational numbers, should be set to zero.

To isolate those terms, we determine eigenvectors of the matrix $\boldsymbol{M}(\epsilon)$ with zero eigenvalues

$$
\boldsymbol{M}(\epsilon) \boldsymbol{\xi}_{a}=0, \quad a=1, . ., N_{0},
$$

and write

$$
\boldsymbol{J}_{m^{2}=0}=\sum_{a=1}^{N}\left(\widetilde{\boldsymbol{\xi}}_{a}^{\dagger} \cdot \mathcal{J}_{0}\right) \boldsymbol{P}(\varepsilon, 0) \boldsymbol{\xi}_{a}
$$

where dual normalized eigenvectors $\left\{\widetilde{\boldsymbol{\xi}}_{a}^{\dagger}\right\}$ are orthogonal to $\left\{\boldsymbol{\xi}_{a}\right\}$, i.e $\widetilde{\boldsymbol{\xi}}_{b}^{\dagger} \cdot \boldsymbol{\xi}_{a}=\delta_{a b}$. The above equation provides the desired results for integrals $\mathcal{J}_{1, . ., 11}$ at $m^{2}=0$.

We emphasize that the algorithm described above can be used to obtain numerical solutions of the differential equations with arbitrary precision. To illustrate this point, we present the results for two integrals that appear in eq. (5.82) at the physical point $m^{2}=0$ 
computed up to at least 15 significant digits through weight six. They read

$$
\begin{aligned}
\left.\mathcal{J}_{9}\right|_{m^{2}=0}= & \int \frac{\mathrm{d} \Phi_{\delta \theta \theta}^{n n n}}{k_{123}^{2}}=-\frac{0.5}{\epsilon}-5-28.17026373260709 \epsilon-119.43143332972728 \epsilon^{2} \\
& -430.4404286909044 \epsilon^{3}-1410.1679482808422 \epsilon^{4} \\
& -4372.111524529197 \epsilon^{5}-13148.701437210732 \epsilon^{6}+\mathcal{O}\left(\epsilon^{7}\right), \\
\left.\mathcal{J}_{10}\right|_{m^{2}=0}= & \int \frac{\mathrm{d} \Phi_{\delta \theta \theta}^{n n n}}{\left(k_{123}^{2}\right)\left(k_{2} \bar{n}\right)}=\frac{0.5}{\varepsilon^{2}}+\frac{5.5}{\varepsilon}+34.34926305180546+170.0583525098628 \epsilon \\
& +758.7443815516605 \epsilon^{2}+3238.222100561864 \epsilon^{3} \\
& +13535.346184323936 \epsilon^{4}+\mathcal{O}\left(\epsilon^{5}\right) .
\end{aligned}
$$

Numerical results for remaining nine integrals shown in eq. (5.82) can be found in the supplementary material. We note that it is possible to reconstruct analytic solutions using the PSLQ algorithm [66] and a basis of transcendental constants. Using FindIntegerNullVector function provided by Mathematica, we test this explicitly for all the integrals in this example and verify the analytic solutions numerically up to 2000 digits.

\section{Conclusion}

In this paper we discussed computation of real-emission integrals for observables that contain Heaviside functions. This is an interesting problem because reverse unitarity [44] cannot be immediately applied to map such integrals onto multi-loop integrals, preventing straightforward use of integration-by-parts identities in such cases.

We discussed a way to re-introduce integration-by-parts technology into the computation of such integrals and showed that the resulting IBP relations have a clear hierarchical structure since, in addition to original integrals, there appear integrals with Heaviside functions replaced with $\delta$-functions. Integrals with $\delta$-functions are, however, much simpler since they can be dealt with using reverse unitarity and, thus, IBP equations for them are self-contained. In addition, IBP relations provide a foundation for deriving differential equations for real-emission master integrals with Heaviside functions that can be solved numerically even if analytic integration becomes too difficult.

We have shown the efficacy of this approach by computing the real-emission contribution to the zero-jettiness soft function at NNLO. We have also discussed several non-trivial contributions to the zero-jettiness soft function at N3LO and used them to illustrate all the different aspects of the proposed computational techniques. We believe that theoretical methods discussed in this paper will be useful for computing other phase-space integrals that involve Heaviside functions. In particular, we hope that their application will allow us to complete the computation of all real-emission contributions to zero-jettiness soft function at N3LO.

\section{Acknowledgments}

We would like to thank Arnd Behring for fruitful discussions and help related to multivariate partial fractioning. We are grateful to Dimitri Colferai for clarifying correspondence 
regarding the soft eikonal function for triple-gluon emission and for providing us with a digital version of the results of ref. [61]. We are indebted to Fabian Lange for his help with Kira.

This research is partially supported by the Deutsche Forschungsgemeinschaft (DFG, German Research Foundation) under grant 396021762 — TRR 257 and by Karlsruhe School of Particle and Astroparticle physics (KSETA). MD is supported by the Excellence Cluster ORIGINS funded by the Deutsche Forschungsgemeinschaft (DFG, German Research Foundation) under Germany's Excellence Strategy — EXC-2094 - 390783311 and by the ERC Starting Grant 949279 HighPHuN.

\section{A Master integrals for $n n n$ contribution to the soft function}

The master integrals that arise in the computation of the $n n n$ contribution to the soft function are defined as follows:

- integrals for $\int \mathrm{d} \Phi_{\theta \theta \theta}^{n n n} \omega_{n \bar{n}}^{(3), a}$ :

$$
\begin{aligned}
I_{1} & =\int \mathrm{d} \Phi_{\delta \delta \delta}^{n n n}, & I_{2} & =\int \frac{\mathrm{d} \Phi_{\delta \delta \delta}^{n n n}}{\left(k_{12} n\right)\left(k_{13} n\right)}, \\
I_{3} & =\int \frac{\mathrm{d} \Phi_{\delta \delta \theta}^{n n n}}{\left(k_{13} \bar{n}\right)}, & I_{4} & =\int \frac{\mathrm{d} \Phi_{\delta \delta \theta}^{n n n}}{\left(k_{123} \bar{n}\right)}, \\
I_{5} & =\int \frac{\mathrm{d} \Phi_{\delta \delta \theta}^{n n n}}{\left(k_{13} n\right)\left(k_{123} \bar{n}\right)}, & I_{6} & =\int \frac{\mathrm{d} \Phi_{\delta \theta \theta}^{n n n}}{\left(k_{123} \bar{n}\right)} .
\end{aligned}
$$

- additional integrals for $\int \mathrm{d} \Phi_{\theta \theta \theta}^{n n n} \omega_{n \bar{n}}^{(3), b}$ :

$$
\begin{aligned}
I_{7} & =\int \frac{\mathrm{d} \Phi_{\delta \delta \theta}^{n n n}}{\left(k_{1} k_{3}\right)\left(k_{3} \bar{n}\right)}, & I_{8} & =\int \frac{\mathrm{d} \Phi_{\delta \delta \theta}^{n n n}}{\left(k_{1} k_{3}\right)\left(k_{3} n\right)\left(k_{13} \bar{n}\right)}, \\
I_{9} & =\int \frac{\mathrm{d} \Phi_{\delta \delta \theta}^{n n n}}{\left(k_{1} k_{3}\right)\left(k_{3} n\right)\left(k_{23} \bar{n}\right)}, & I_{10} & =\int \frac{\mathrm{d} \Phi_{\delta \delta \theta}^{n n n}}{\left(k_{1} k_{3}\right)\left(k_{12} n\right)\left(k_{13} \bar{n}\right)}, \\
I_{11} & =\int \frac{\mathrm{d} \Phi_{\delta \delta \theta}^{n n n}}{\left(k_{1} k_{3}\right)\left(k_{3} n\right)\left(k_{123} \bar{n}\right)}, & I_{12} & =\int \frac{\mathrm{d} \Phi_{\delta \theta \delta}^{n n n}}{\left(k_{1} k_{2}\right)\left(k_{12} n\right)\left(k_{2} \bar{n}\right)\left(k_{13} n\right)}, \\
I_{13} & =\int \frac{\mathrm{d} \Phi_{\delta \theta \delta}^{n n n}}{\left(k_{1} k_{2}\right)\left(k_{12} n\right)\left(k_{2} \bar{n}\right)\left(k_{123} \bar{n}\right)}, & I_{14} & =\int \frac{\mathrm{d} \Phi_{\theta \delta \delta}^{n n n}}{\left(k_{1} k_{3}\right)\left(k_{12} n\right)\left(k_{13} \bar{n}\right)}, \\
I_{15} & =\int \frac{\mathrm{d} \Phi_{\theta \delta \delta}^{n n n}}{\left(k_{1} k_{2}\right)\left(k_{12} n\right)\left(k_{13} \bar{n}\right)}, & I_{16} & =\int \frac{\mathrm{d} \Phi_{\delta \theta \theta}^{n n n}}{\left(k_{1} k_{2}\right)\left(k_{23} \bar{n}\right)}, \\
I_{17} & =\int \frac{\mathrm{d} \Phi_{\delta \theta \theta}^{n n n}}{\left(k_{1} k_{2}\right)\left(k_{2} \bar{n}\right)\left(k_{13} \bar{n}\right)}, & I_{18} & =\int \frac{\mathrm{d} \Phi_{\delta \theta \theta}^{n n n}}{\left(k_{1} k_{2}\right)\left(k_{2} \bar{n}\right)\left(k_{123} \bar{n}\right)}, \\
I_{19} & =\int \frac{\mathrm{d} \Phi_{\delta \theta \theta}^{n n n}}{\left(k_{1} k_{2}\right)\left(k_{2} n\right)\left(k_{123} \bar{n}\right)}, & I_{20} & =\int \frac{\mathrm{d} \Phi_{\delta \theta \theta}^{n n n}}{\left(k_{1} k_{3}\right)\left(k_{12} n\right)\left(k_{123} \bar{n}\right)}, \\
I_{21} & =\int \frac{\mathrm{d} \Phi_{\delta \theta \theta}^{n n n}}{\left(k_{1} k_{2}\right)\left(k_{23} n\right)\left(k_{123} \bar{n}\right)}, & I_{22} & =\int \frac{\mathrm{d} \Phi_{\theta \delta \theta}^{n n n}}{\left(k_{1} k_{3}\right)\left(k_{12} n\right)\left(k_{123} \bar{n}\right)} .
\end{aligned}
$$


- additional integrals for $\int \mathrm{d} \Phi_{\theta \theta \theta}^{n n n} \omega_{n \bar{n}}^{(3), c}$ :

$$
\begin{array}{rlrl}
I_{23} & =\int \frac{\mathrm{d} \Phi_{\theta \delta \delta}^{n n n}}{\left(k_{1} k_{2}\right)\left(k_{1} k_{3}\right)}, & I_{24} & =\int \frac{\mathrm{d} \Phi_{\theta \delta \delta}^{n n n}}{\left(k_{1} k_{2}\right)\left(k_{1} k_{3}\right)\left(k_{12} \bar{n}\right)}, \\
I_{25} & =\int \frac{\mathrm{d} \Phi_{\theta \delta \delta}^{n n n}}{\left(k_{1} k_{2}\right)\left(k_{1} k_{3}\right)\left(k_{123} \bar{n}\right)}, & I_{26} & =\int \frac{\mathrm{d} \Phi_{\theta \delta \delta}^{n n n}}{\left(k_{1} k_{2}\right)\left(k_{1} k_{3}\right)\left(k_{12} n\right)\left(k_{13} \bar{n}\right)}, \\
I_{27} & =\int \frac{\mathrm{d} \Phi_{\theta \delta \delta}^{n n n}}{\left(k_{1} k_{2}\right)\left(k_{1} k_{3}\right)\left(k_{12} n\right)\left(k_{123} \bar{n}\right)}, & I_{28} & =\int \frac{\mathrm{d} \Phi_{\theta \delta \theta}^{n n n}}{\left(k_{1} k_{2}\right)\left(k_{1} k_{3}\right)\left(k_{3} \bar{n}\right)}, \\
I_{29} & =\int \frac{\mathrm{d} \Phi_{\theta \delta \theta}^{n n n}}{\left(k_{1} k_{2}\right)\left(k_{1} k_{3}\right)\left(k_{12} \bar{n}\right)\left(k_{3} \bar{n}\right)}, & I_{30} & =\int \frac{\mathrm{d} \Phi_{\theta \delta \theta}^{n n n}}{\left(k_{1} k_{2}\right)\left(k_{1} k_{3}\right)\left(k_{12} n\right)\left(k_{13} \bar{n}\right)}, \\
I_{31} & =\int \frac{\mathrm{d} \Phi_{\theta \delta \theta}^{n n n}}{\left(k_{1} k_{2}\right)\left(k_{1} k_{3}\right)\left(k_{123} \bar{n}\right)\left(k_{3} \bar{n}\right)}, & I_{32} & =\int \frac{\mathrm{d} \Phi_{\theta \delta \theta}^{n n n}}{\left(k_{1} k_{2}\right)\left(k_{1} k_{3}\right)\left(k_{3} n\right)\left(k_{123} \bar{n}\right)}, \\
I_{33} & =\int \frac{\mathrm{d} \Phi_{\theta \delta \theta}^{n n n}}{\left(k_{1} k_{2}\right)\left(k_{1} k_{3}\right)\left(k_{12} n\right)\left(k_{123} \bar{n}\right)}, & I_{34} & =\int \frac{\mathrm{d} \Phi_{\theta \theta \theta}^{n n n}}{\left(k_{1} k_{2}\right)\left(k_{1} k_{3}\right)\left(k_{3} n\right)\left(k_{123} \bar{n}\right)^{2}}, \\
I_{35} & =\int \frac{\mathrm{d} \Phi_{\delta \theta \theta}^{n n n}}{\left(k_{1} k_{2}\right)\left(k_{1} k_{3}\right)\left(k_{2} n\right)\left(k_{123} \bar{n}\right)\left(k_{3} \bar{n}\right)}, & I_{36} & =\int \frac{\mathrm{d} \Phi_{\delta \theta \theta}^{n n n}}{\left(k_{1} k_{2}\right)\left(k_{1} k_{3}\right)\left(k_{12} n\right)\left(k_{2} \bar{n}\right)\left(k_{13} \bar{n}\right)}, \\
I_{37} & =\int \frac{\mathrm{d} \Phi_{\delta \theta \theta}^{n n n}}{\left(k_{1} k_{2}\right)\left(k_{1} k_{3}\right)\left(k_{12} n\right)\left(k_{123} \bar{n}\right)\left(k_{2} \bar{n}\right)}, & I_{38} & =\int \frac{\mathrm{d} \Phi_{\theta \theta \delta}^{n n n}}{\left(k_{1} k_{2}\right)\left(k_{1} k_{3}\right)\left(k_{12} n\right)\left(k_{2} \bar{n}\right)\left(k_{13} \bar{n}\right)}, \\
I_{39}=\int \frac{k_{\theta \delta}}{\left(k_{1} k_{2}\right)\left(k_{1} k_{3}\right)\left(k_{12} n\right)\left(k_{123} \bar{n}\right)\left(k_{2} \bar{n}\right)} . & &
\end{array}
$$

- additional integral for $\int \mathrm{d} \Phi_{\theta \theta \theta}^{n n n} \omega_{n \bar{n}}^{(3), d}$ without $1 / k_{123}^{2}$ propagator:

$$
I_{40}=\int \frac{\mathrm{d} \Phi_{\theta \delta \theta}^{n n n}}{\left(k_{1} k_{3}\right)\left(k_{1} n\right)\left(k_{123} \bar{n}\right)\left(k_{3} \bar{n}\right)}
$$

- additional integrals for $\int \mathrm{d} \Phi_{\theta \theta \theta}^{n n n} \omega_{n \bar{n}}^{(3), d}$ with $1 / k_{123}^{2}$ propagator:

$$
\begin{aligned}
I_{41} & =\int \frac{\mathrm{d} \Phi_{\delta \delta \delta}^{n n n}}{k_{123}^{2}\left(k_{12} n\right)}, & I_{42} & =\int \frac{\mathrm{d} \Phi_{\delta \delta \delta}^{n n n}}{k_{123}^{2}\left(k_{1} n\right)\left(k_{2} n\right)}, \\
I_{43} & =\int \frac{\mathrm{d} \Phi_{\delta \delta \theta}^{n n n}}{k_{123}^{2}}, & I_{44} & =\int \frac{\mathrm{d} \Phi_{\delta \delta \theta}^{n n n}}{k_{123}^{2}\left(k_{3} \bar{n}\right)}, \\
I_{45} & =\int \frac{\mathrm{d} \Phi_{\delta \delta \theta}^{n n n}}{k_{123}^{2}\left(k_{13} \bar{n}\right)}, & I_{46} & =\int \frac{\mathrm{d} \Phi_{\delta \delta \theta}^{n n n}}{k_{123}^{2}\left(k_{23} n\right)}, \\
I_{47} & =\int \frac{\mathrm{d} \Phi_{\delta \delta \theta}^{n n n}}{k_{123}^{2}\left(k_{13} \bar{n}\right)^{2}}, & I_{48} & =\int \frac{\mathrm{d} \Phi_{\delta \delta \theta}^{n n n}}{k_{123}^{2}\left(k_{1} n\right)\left(k_{3} \bar{n}\right)}, \\
I_{49} & =\int \frac{\mathrm{d} \Phi_{\delta \delta \theta}^{n n n}}{k_{123}^{2}\left(k_{1} k_{3}\right)\left(k_{3} \bar{n}\right)}, & I_{50} & =\int \frac{\mathrm{d} \Phi_{\delta \delta \theta}^{n n n}}{k_{123}^{2}\left(k_{1} k_{3}\right)\left(k_{3} n\right)}, \\
I_{51} & =\int \frac{\mathrm{d} \Phi_{\delta \delta \theta}^{n n n}}{k_{123}^{2}\left(k_{13} \bar{n}\right)\left(k_{2} n\right)}, & I_{52} & =\int \frac{\mathrm{d} \Phi_{\delta \delta \theta}^{n n n}}{k_{123}^{2}\left(k_{13} \bar{n}\right)\left(k_{1} n\right)}, \\
I_{53} & =\int \frac{\mathrm{d} \Phi_{\delta \delta \theta}^{n n n}}{k_{123}^{2}\left(k_{1} k_{3}\right)\left(k_{23} \bar{n}\right)}, & I_{54} & =\int \frac{\mathrm{d} \Phi_{\delta \delta \theta}^{n n n}}{k_{123}^{2}\left(k_{12} n\right)\left(k_{1} k_{3}\right)}, \\
I_{55} & =\int \frac{\mathrm{d} \Phi_{\delta \delta \theta}^{n n n}}{k_{123}^{2}\left(k_{1} k_{3}\right)\left(k_{23} n\right)}, & I_{56} & =\int \frac{\mathrm{d} \Phi_{\delta \delta \theta}^{n n n}}{k_{123}^{2}\left(k_{123} \bar{n}\right)\left(k_{1} n\right)}, \\
I_{57} & =\int \frac{\mathrm{d} \Phi_{\delta \delta \theta}^{n n n}}{k_{123}^{2}\left(k_{123} \bar{n}\right)\left(k_{3} n\right)}, & I_{58} & =\int \frac{\mathrm{d} \Phi_{\delta \delta \theta}^{n n n}}{k_{123}^{2}\left(k_{123} \bar{n}\right)\left(k_{13} n\right)}, \\
I_{59} & =\int \frac{\mathrm{d} \Phi_{\delta \delta \theta}^{n n n}}{k_{123}^{2}\left(k_{1} k_{3}\right)\left(k_{23} \bar{n}\right)^{2}}, & I_{60} & =\int \frac{\mathrm{d} \Phi_{\delta \delta \theta}^{n n n}}{k_{123}^{2}\left(k_{123} \bar{n}\right)^{2}\left(k_{13} n\right)}, \\
I_{61} & =\int \frac{\mathrm{d} \Phi_{\delta \delta \theta}^{n n n}}{k_{123}^{2}\left(k_{12} n\right)\left(k_{1} k_{3}\right)\left(k_{3} \bar{n}\right)}, & I_{62} & =\int \frac{\mathrm{d} \Phi_{\delta \delta \theta}^{n n n}}{k_{123}^{2}\left(k_{123} \bar{n}\right)\left(k_{1} n\right)\left(k_{3} n\right)},
\end{aligned}
$$




$$
\begin{aligned}
& I_{63}=\int \frac{\mathrm{d} \Phi_{\delta \delta \theta}^{n n n}}{k_{123}^{2}\left(k_{123} \bar{n}\right)\left(k_{1} k_{3}\right)\left(k_{3} n\right)}, \quad \quad I_{64}=\int \frac{\mathrm{d} \Phi_{\delta \delta \theta}^{n n n}}{k_{123}^{2}\left(k_{123} \bar{n}\right)\left(k_{1} k_{3}\right)\left(k_{3} \bar{n}\right)}, \\
& I_{65}=\int \frac{\mathrm{d} \Phi_{\delta \delta \theta}^{n n n}}{k_{123}^{2}\left(k_{123} \bar{n}\right)\left(k_{13} n\right)\left(k_{1} n\right)}, \quad \quad I_{66}=\int \frac{\mathrm{d} \Phi_{\delta \delta \theta}^{n n n}}{k_{123}^{2}\left(k_{123} \bar{n}\right)\left(k_{1} k_{3}\right)\left(k_{23} n\right)}, \\
& I_{67}=\int \frac{\mathrm{d} \Phi_{\delta \theta \theta}^{n n n}}{k_{123}^{2}\left(k_{2} \bar{n}\right)}, \quad \quad I_{68}=\int \frac{\mathrm{d} \Phi_{\delta \theta \theta}^{n n n}}{k_{123}^{2}\left(k_{13} \bar{n}\right)}, \\
& I_{69}=\int \frac{\mathrm{d} \Phi_{\delta \theta \theta}^{n n n}}{k_{123}^{2}\left(k_{13} \bar{n}\right)\left(k_{1} k_{2}\right)}, \quad \quad I_{70}=\int \frac{\mathrm{d} \Phi_{\delta \theta \theta}^{n n n}}{k_{123}^{2}\left(k_{12} n\right)\left(k_{3} \bar{n}\right)}, \\
& I_{71}=\int \frac{\mathrm{d} \Phi_{\delta \theta \theta}^{n n n}}{k_{123}^{2}\left(k_{123} \bar{n}\right)\left(k_{2} n\right)}, \quad \quad I_{72}=\int \frac{\mathrm{d} \Phi_{\delta \theta \theta}^{n n n}}{k_{123}^{2}\left(k_{123} \bar{n}\right)\left(k_{12} n\right)}, \\
& I_{73}=\int \frac{\mathrm{d} \Phi_{\delta \theta \theta}^{n n n}}{k_{123}^{2}\left(k_{1} k_{2}\right)\left(k_{2} \bar{n}\right)\left(k_{3} \bar{n}\right)}, \quad I_{74}=\int \frac{\mathrm{d} \Phi_{\delta \theta \theta}^{n n n}}{k_{123}^{2}\left(k_{1} k_{2}\right)\left(k_{2} n\right)\left(k_{3} \bar{n}\right)}, \\
& I_{75}=\int \frac{\mathrm{d} \Phi_{\delta \theta \theta}^{n n n}}{k_{123}^{2}\left(k_{13} \bar{n}\right)\left(k_{2} n\right)\left(k_{2} \bar{n}\right)}, \quad \quad I_{76}=\int \frac{\mathrm{d} \Phi_{\delta \theta \theta}^{n n n}}{k_{123}^{2}\left(k_{13} \bar{n}\right)\left(k_{1} k_{2}\right)\left(k_{2} n\right)}, \\
& I_{77}=\int \frac{\mathrm{d} \Phi_{\delta \theta \theta}^{n n n}}{k_{123}^{2}\left(k_{1} k_{2}\right)\left(k_{23} \bar{n}\right)\left(k_{2} \bar{n}\right)}, \quad \quad I_{78}=\int \frac{\mathrm{d} \Phi_{\delta \theta \theta}^{n n n}}{k_{123}^{2}\left(k_{123} \bar{n}\right)\left(k_{2} n\right)\left(k_{2} \bar{n}\right)}, \\
& I_{79}=\int \frac{\mathrm{d} \Phi_{\delta \theta \theta}^{n n n}}{k_{123}^{2}\left(k_{123} \bar{n}\right)\left(k_{12} n\right)\left(k_{1} k_{3}\right)}, \quad \quad I_{80}=\int \frac{\mathrm{d} \Phi_{\theta \delta \theta}^{n n n}}{k_{123}^{2}\left(k_{12} n\right)\left(k_{1} k_{3}\right)\left(k_{3} \bar{n}\right)}, \\
& I_{81}=\int \frac{\mathrm{d} \Phi_{\theta \delta \theta}^{n n n}}{k_{123}^{2}\left(k_{1} k_{3}\right)\left(k_{1} n\right)\left(k_{23} \bar{n}\right)}, \quad I_{82}=\int \frac{\mathrm{d} \Phi_{\theta \delta \theta}^{n n n}}{k_{123}^{2}\left(k_{1} k_{3}\right)\left(k_{1} n\right)\left(k_{23} \bar{n}\right)^{2}}, \\
& I_{83}=\int \frac{\mathrm{d} \Phi_{\delta \theta \theta}^{n n n}}{k_{123}^{2}\left(k_{123} \bar{n}\right)\left(k_{1} k_{3}\right)\left(k_{2} n\right)\left(k_{3} \bar{n}\right)}, \quad I_{84}=\int \frac{\mathrm{d} \Phi_{\delta \theta \theta}^{n n n}}{k_{123}^{2}\left(k_{123} \bar{n}\right)\left(k_{13} n\right)\left(k_{1} k_{2}\right)\left(k_{2} \bar{n}\right)}, \\
& I_{85}=\int \frac{\mathrm{d} \Phi_{\delta \theta \theta}^{n n n}}{k_{123}^{2}\left(k_{123} \bar{n}\right)\left(k_{1} k_{3}\right)\left(k_{23} n\right)\left(k_{2} n\right)}, \quad I_{86}=\int \frac{\mathrm{d} \Phi_{\theta \delta \theta}^{n n n}}{k_{123}^{2}\left(k_{123} \bar{n}\right)\left(k_{1} k_{3}\right)\left(k_{1} \bar{n}\right)\left(k_{23} n\right)} .
\end{aligned}
$$

- additional integral for $\int \mathrm{d} \Phi_{\theta \theta \theta}^{n n n} \omega_{n \bar{n}}^{(3), d}$ with $1 / k_{123}^{2}$ propagator and $1 / \nu$ behaviour:

$$
J_{\nu}^{(d)}=\int \frac{\mathrm{d} \Phi_{\theta \delta \theta}^{n n n}\left(k_{1} n\right)^{\nu}\left(k_{2} n\right)^{\nu}\left(k_{3} n\right)^{\nu}}{k_{123}^{2}\left(k_{1} k_{3}\right)\left(k_{1} n\right)\left(k_{12} \bar{n}\right)\left(k_{3} \bar{n}\right)} .
$$

\section{B Matrices for the differential equations in section 5.4.2}

In section 5.4.2, we discussed an example of a differential equation w.r.t. the auxiliary mass parameter. The differential equation for eleven selected integrals is given in eq. (5.83); it involves five eleven-by-eleven matrices whose explicit form is shown below.

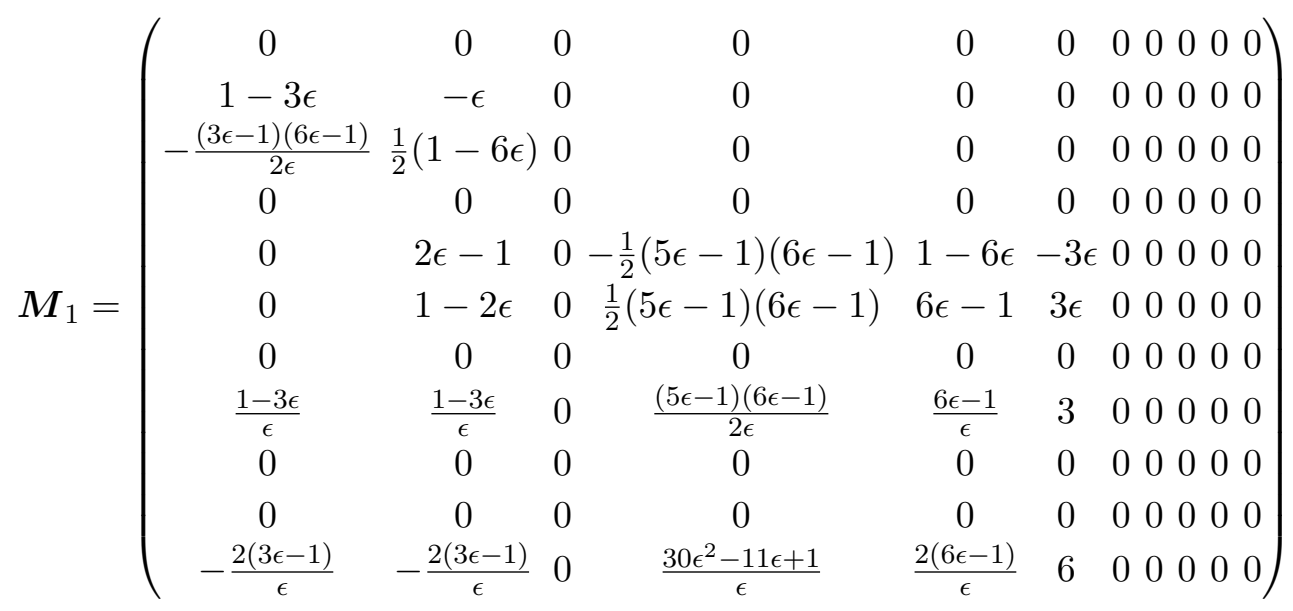




$$
\begin{aligned}
& \boldsymbol{M}_{2}=\left(\begin{array}{ccccccccccc}
0 & 0 & 0 & 0 & 0 & 0 & 0 & 0 & 0 & 0 & 0 \\
0 & 0 & 0 & 0 & 0 & 0 & 0 & 0 & 0 & 0 & 0 \\
0 & 0 & 0 & 0 & 0 & 0 & 0 & 0 & 0 & 0 & 0 \\
0 & 0 & 0 & 0 & 0 & 0 & 0 & 0 & 0 & 0 & 0 \\
0 & 0 & 0 & 0 & 0 & 0 & 0 & 0 & 0 & 0 & 0 \\
0 & 0 & 0 & 0 & 0 & 0 & 0 & 0 & 0 & 0 & 0 \\
0 & 0 & 0 & 0 & 0 & 0 & 0 & 0 & 0 & 0 & 0 \\
\frac{3 \epsilon-1}{\epsilon} & \frac{3 \epsilon \epsilon-1}{\epsilon} & \frac{1}{4} & -\frac{(5 \epsilon-1)(6 \epsilon-1)}{2 \epsilon} & -\frac{3(6 \epsilon-1)}{8 \epsilon} & -3 & 0 & -3 \epsilon & 0 & 0 & 0 \\
0 & 0 & 0 & 0 & 0 & 0 & 0 & 0 & 0 & 0 & 0 \\
0 & 0 & 0 & 0 & 0 & 0 & 0 & 0 & 0 & 0 & 0 \\
\frac{2(3 \epsilon-1)(7 \epsilon-2)}{\epsilon(5 \epsilon-2)} & \frac{11 \epsilon-4}{2 \epsilon} & 0 & \frac{-238 \epsilon^{3}+157 \epsilon^{2}-32 \epsilon+2}{\epsilon(5 \epsilon-2)} \frac{-25 \epsilon^{2}+11 \epsilon-1}{\epsilon(5 \epsilon-2)} & \frac{34-97 \epsilon}{2(5 \epsilon-2)} & \frac{7 \epsilon}{2} & \frac{1}{2}(2 \epsilon-1) & 4(2 \epsilon-1)(3 \epsilon-1)-\frac{1}{2}(2 \epsilon-1)(6 \epsilon-1) \frac{1}{2}(1-8 \epsilon)
\end{array}\right) \\
& \text { 点 } \\
& \boldsymbol{M}_{3}=\left(\begin{array}{ccccccccccc}
0 & 0 & 0 & 0 & 0 & 0 & 0 & 0 & 0 & 0 & 0 \\
3 \epsilon-1 & -2 \epsilon & 0 & 0 & 0 & 0 & 0 & 0 & 0 & 0 & 0 \\
\frac{(3 \epsilon-1)(6 \epsilon-1)}{2 \epsilon} & \frac{1}{2}(6 \epsilon-1) & \frac{1}{2}(-6 \epsilon-1) & 0 & 0 & 0 & 0 & 0 & 0 & 0 & 0 \\
0 & 0 & 0 & 0 & 0 & 0 & 0 & 0 & 0 & 0 & 0 \\
0 & 1-2 \epsilon & 0 & \frac{1}{2}(5 \epsilon-1)(6 \epsilon-1) & \frac{1}{2}(-4 \epsilon-1) & 3 \epsilon & 0 & 0 & 0 & 0 & 0 \\
0 & \frac{1}{2}(2 \epsilon-1) & 0 & -\frac{1}{2}(5 \epsilon-1)(6 \epsilon-1) & 0 & -4 \epsilon & 0 & 0 & 0 & 0 & 0 \\
0 & \frac{2 \epsilon-1}{2 \epsilon} & 0 & 0 & 0 & -2 & -3 \epsilon & 0 & 0 & 0 & 0 \\
0 & 0 & 0 & 0 & 0 & 0 & 0 & 0 & 0 & 0 & 0 \\
\frac{1}{2-5 \epsilon} & 0 & 0 & \frac{(4 \epsilon-1)^{2}}{(3 \epsilon-1)(5 \epsilon-2)} & 0 & 0 & 0 & 0 & 1-2 \epsilon & 0 & 0 \\
0 & 0 & 0 & 0 & 0 & 0 & 0 & 0 & 0 & \frac{1}{2}(1-6 \epsilon) & \frac{1}{2} \\
-\frac{4(3 \epsilon-1)}{5 \epsilon-2} & 0 & 0 & \frac{4(4 \epsilon-1)^{2}}{5 \epsilon-2} & 0 & 0 & 0 & 0-4(2 \epsilon-1)(3 \epsilon-1) \frac{1}{2}(2 \epsilon-1)(6 \epsilon-1) \frac{1}{2}(1-2 \epsilon)
\end{array}\right)
\end{aligned}
$$




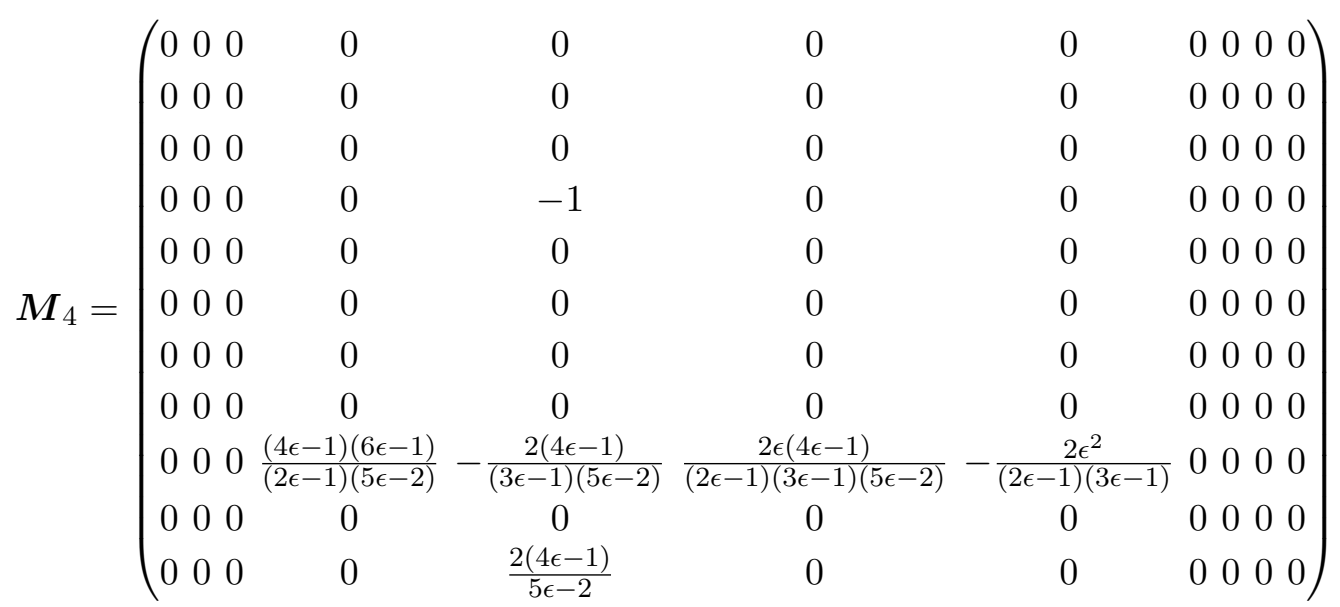

$$
\boldsymbol{M}_{5}=\left(\begin{array}{llllllllllll}
0 & 0 & 0 & 0 & 0 & 0 & 0 & 0 & 0 & 0 & 0 \\
0 & 0 & 0 & 0 & 0 & 0 & 0 & 0 & 0 & 0 & 0 \\
0 & 0 & 0 & 0 & 0 & 0 & 0 & 0 & 0 & 0 & 0 \\
0 & 0 & 0 & 0 & 0 & 0 & 0 & 0 & 0 & 0 & 0 \\
0 & 0 & 0 & 0 & 0 & 0 & 0 & 0 & 0 & 0 & 0 \\
0 & 0 & 0 & 0 & 0 & 0 & 0 & 0 & 0 & 0 & 0 \\
0 & 0 & 0 & 0 & 0 & 0 & 0 & 0 & 0 & 0 & 0 \\
0 & 0 & 0 & 0 & 0 & 0 & 0 & 0 & 0 & 0 & 0 \\
0 & 0 & 0 & 0 & 2(4 \epsilon-1) & 0 & 0 & 0 & 0 & 0 & 0 & 0 \\
0 & 0 & 0 & 0 & 0 & 0 & 0 & 0 & 0 & 0 & 0 \\
0 & 0 & 0 & 0 & 0 & 0 & 0 & 0 & 0 & 0 & 0
\end{array}\right)
$$

Open Access. This article is distributed under the terms of the Creative Commons Attribution License (CC-BY 4.0), which permits any use, distribution and reproduction in any medium, provided the original author(s) and source are credited.

\section{References}

[1] J.C. Collins, D.E. Soper and G.F. Sterman, Factorization of Hard Processes in QCD, Adv. Ser. Direct. High Energy Phys. 5 (1989) 1 [hep-ph/0409313] [InSPIRE].

[2] C.W. Bauer, S. Fleming and M.E. Luke, Summing Sudakov logarithms in $B \rightarrow X_{s \gamma}$ in effective field theory, Phys. Rev. D 63 (2000) 014006 [hep-ph/0005275] [INSPIRE].

[3] C.W. Bauer, S. Fleming, D. Pirjol and I.W. Stewart, An effective field theory for collinear and soft gluons: Heavy to light decays, Phys. Rev. D 63 (2001) 114020 [hep-ph/0011336] [INSPIRE].

[4] C.W. Bauer and I.W. Stewart, Invariant operators in collinear effective theory, Phys. Lett. B 516 (2001) 134 [hep-ph/0107001] [INSPIRE].

[5] C.W. Bauer, D. Pirjol and I.W. Stewart, Soft collinear factorization in effective field theory, Phys. Rev. D 65 (2002) 054022 [hep-ph/0109045] [INSPIRE].

[6] C.W. Bauer, S. Fleming, D. Pirjol, I.Z. Rothstein and I.W. Stewart, Hard scattering factorization from effective field theory, Phys. Rev. D 66 (2002) 014017 [hep-ph/0202088] [INSPIRE]. 
[7] S. Catani and M. Grazzini, An NNLO subtraction formalism in hadron collisions and its application to Higgs boson production at the LHC, Phys. Rev. Lett. 98 (2007) 222002 [hep-ph/0703012] [INSPIRE].

[8] R. Bonciani, S. Catani, M. Grazzini, H. Sargsyan and A. Torre, The $q_{T}$ subtraction method for top quark production at hadron colliders, Eur. Phys. J. C $\mathbf{7 5}$ (2015) 581 [arXiv:1508.03585] [INSPIRE].

[9] M. Grazzini, S. Kallweit and M. Wiesemann, Fully differential NNLO computations with MATRIX, Eur. Phys. J. C 78 (2018) 537 [arXiv:1711.06631] [inSPIRE].

[10] S. Catani, S. Devoto, M. Grazzini, S. Kallweit, J. Mazzitelli and H. Sargsyan, Top-quark pair hadroproduction at next-to-next-to-leading order in QCD, Phys. Rev. D 99 (2019) 051501 [arXiv: 1901.04005] [INSPIRE].

[11] S. Catani, S. Devoto, M. Grazzini, S. Kallweit and J. Mazzitelli, Top-quark pair production at the LHC: Fully differential QCD predictions at NNLO, JHEP 07 (2019) 100 [arXiv: 1906.06535] [INSPIRE].

[12] S. Kallweit, V. Sotnikov and M. Wiesemann, Triphoton production at hadron colliders in NNLO QCD, Phys. Lett. B 812 (2021) 136013 [arXiv:2010.04681] [INSPIRE].

[13] S. Catani, S. Devoto, M. Grazzini, S. Kallweit and J. Mazzitelli, Bottom-quark production at hadron colliders: fully differential predictions in NNLO QCD, JHEP 03 (2021) 029 [arXiv:2010.11906] [INSPIRE].

[14] S. Catani, I. Fabre, M. Grazzini and S. Kallweit, $t \bar{t} H$ production at NNLO: the flavour off-diagonal channels, Eur. Phys. J. C 81 (2021) 491 [arXiv:2102.03256] [INSPIRE].

[15] R. Boughezal, C. Focke, X. Liu and F. Petriello, $W$-boson production in association with a jet at next-to-next-to-leading order in perturbative QCD, Phys. Rev. Lett. 115 (2015) 062002 [arXiv: 1504.02131] [INSPIRE].

[16] R. Boughezal, C. Focke, W. Giele, X. Liu and F. Petriello, Higgs boson production in association with a jet at NNLO using jettiness subtraction, Phys. Lett. B 748 (2015) 5 [arXiv: 1505.03893] [INSPIRE].

[17] J. Gaunt, M. Stahlhofen, F.J. Tackmann and J.R. Walsh, N-jettiness Subtractions for NNLO QCD Calculations, JHEP 09 (2015) 058 [arXiv: 1505.04794] [INSPIRE].

[18] R. Boughezal et al., Color singlet production at NNLO in MCFM, Eur. Phys. J. C 77 (2017) 7 [arXiv: 1605.08011] [INSPIRE].

[19] I.W. Stewart, F.J. Tackmann and W.J. Waalewijn, Factorization at the LHC: From PDFs to Initial State Jets, Phys. Rev. D 81 (2010) 094035 [arXiv:0910.0467] [INSPIRE].

[20] I.W. Stewart, F.J. Tackmann and W.J. Waalewijn, N-Jettiness: An Inclusive Event Shape to Veto Jets, Phys. Rev. Lett. 105 (2010) 092002 [arXiv:1004.2489] [INSPIRE].

[21] P.F. Monni, T. Gehrmann and G. Luisoni, Two-Loop Soft Corrections and Resummation of the Thrust Distribution in the Dijet Region, JHEP 08 (2011) 010 [arXiv:1105.4560] [INSPIRE].

[22] R. Kelley, M.D. Schwartz, R.M. Schabinger and H.X. Zhu, The two-loop hemisphere soft function, Phys. Rev. D 84 (2011) 045022 [arXiv: 1105.3676] [INSPIRE].

[23] A. Hornig, C. Lee, I.W. Stewart, J.R. Walsh and S. Zuberi, Non-global Structure of the $\mathcal{O}\left(\alpha_{s}^{2}\right)$ Dijet Soft Function, JHEP 08 (2011) 054 [Erratum ibid. 10 (2017) 101] [arXiv:1105.4628] [INSPIRE]. 
[24] D. Baranowski, NNLO zero-jettiness beam and soft functions to higher orders in the dimensional-regularization parameter $\epsilon$, Eur. Phys. J. C 80 (2020) 523 [arXiv: 2004.03285] [INSPIRE].

[25] J.R. Gaunt, M. Stahlhofen and F.J. Tackmann, The Quark Beam Function at Two Loops, JHEP 04 (2014) 113 [arXiv: 1401.5478] [INSPIRE].

[26] J. Gaunt, M. Stahlhofen and F.J. Tackmann, The Gluon Beam Function at Two Loops, JHEP 08 (2014) 020 [arXiv: 1405.1044] [INSPIRE].

[27] R. Boughezal, F. Petriello, U. Schubert and H. Xing, Spin-dependent quark beam function at NNLO, Phys. Rev. D 96 (2017) 034001 [arXiv:1704.05457] [inSPIRE].

[28] K. Melnikov, R. Rietkerk, L. Tancredi and C. Wever, Double-real contribution to the quark beam function at $N^{3} L O Q C D$, JHEP 02 (2019) 159 [arXiv:1809.06300] [INSPIRE].

[29] K. Melnikov, R. Rietkerk, L. Tancredi and C. Wever, Triple-real contribution to the quark beam function in QCD at next-to-next-to-next-to-leading order, JHEP 06 (2019) 033 [arXiv: 1904.02433] [INSPIRE].

[30] A. Behring, K. Melnikov, R. Rietkerk, L. Tancredi and C. Wever, Quark beam function at next-to-next-to-next-to-leading order in perturbative $Q C D$ in the generalized large- $N_{c}$ approximation, Phys. Rev. D 100 (2019) 114034 [arXiv:1910.10059] [INSPIRE].

[31] M.A. Ebert, B. Mistlberger and G. Vita, Collinear expansion for color singlet cross sections, JHEP 09 (2020) 181 [arXiv:2006.03055] [INSPIRE].

[32] M.A. Ebert, B. Mistlberger and G. Vita, N-jettiness beam functions at $N^{3} L O$, JHEP 09 (2020) 143 [arXiv: 2006.03056] [INSPIRE].

[33] R. Boughezal, X. Liu and F. Petriello, $N$-jettiness soft function at next-to-next-to-leading order, Phys. Rev. D 91 (2015) 094035 [arXiv: 1504.02540] [InSPIRE].

[34] H.T. Li and J. Wang, Next-to-Next-to-Leading Order N-Jettiness Soft Function for One Massive Colored Particle Production at Hadron Colliders, JHEP 02 (2017) 002 [arXiv: 1611.02749] [INSPIRE].

[35] S. Alioli, A. Broggio and M.A. Lim, Zero-jettiness resummation for top-quark pair production at the LHC, JHEP 01 (2022) 066 [arXiv:2111.03632] [INSPIRE].

[36] J.M. Campbell, R.K. Ellis, R. Mondini and C. Williams, The NNLO QCD soft function for 1-jettiness, Eur. Phys. J. C 78 (2018) 234 [arXiv:1711.09984] [INSPIRE].

[37] Y. Li and H.X. Zhu, Bootstrapping Rapidity Anomalous Dimensions for Transverse-Momentum Resummation, Phys. Rev. Lett. 118 (2017) 022004 [arXiv: 1604.01404] [INSPIRE].

[38] R. Angeles-Martinez, M. Czakon and S. Sapeta, NNLO soft function for top quark pair production at small transverse momentum, JHEP 10 (2018) 201 [arXiv:1809.01459] [INSPIRE].

[39] M.-x. Luo, T.-Z. Yang, H.X. Zhu and Y.J. Zhu, Quark Transverse Parton Distribution at the Next-to-Next-to-Next-to-Leading Order, Phys. Rev. Lett. 124 (2020) 092001 [arXiv: 1912.05778] [INSPIRE].

[40] M.A. Ebert, B. Mistlberger and G. Vita, Transverse momentum dependent PDFs at $N^{3} L O$, JHEP 09 (2020) 146 [arXiv: 2006. 05329] [INSPIRE].

[41] G. Bell, R. Rahn and J. Talbert, Generic dijet soft functions at two-loop order: correlated emissions, JHEP 07 (2019) 101 [arXiv:1812.08690] [INSPIRE]. 
[42] A. Basdew-Sharma, F. Herzog, S. Schrijnder van Velzen and W.J. Waalewijn, One-loop jet functions by geometric subtraction, JHEP 10 (2020) 118 [arXiv:2006.14627] [INSPIRE].

[43] K.G. Chetyrkin and F.V. Tkachov, Integration by Parts: The Algorithm to Calculate B-functions in 4 Loops, Nucl. Phys. B 192 (1981) 159 [INSPIRE].

[44] C. Anastasiou and K. Melnikov, Higgs boson production at hadron colliders in NNLO QCD, Nucl. Phys. B 646 (2002) 220 [hep-ph/0207004] [INSPIRE].

[45] F. Caola, M. Delto, H. Frellesvig and K. Melnikov, The double-soft integral for an arbitrary angle between hard radiators, Eur. Phys. J. C 78 (2018) 687 [arXiv:1807.05835] [InSPIRE].

[46] M. Delto and K. Melnikov, Integrated triple-collinear counter-terms for the nested soft-collinear subtraction scheme, JHEP 05 (2019) 148 [arXiv: 1901.05213] [INSPIRE].

[47] W. Bizoń and M. Delto, Analytic double-soft integrated subtraction terms for two massive emitters in a back-to-back kinematics, JHEP 07 (2020) 011 [arXiv:2004.01663] [INSPIRE].

[48] A.V. Smirnov, Algorithm FIRE - Feynman Integral REduction, JHEP 10 (2008) 107 [arXiv:0807.3243] [INSPIRE].

[49] A.V. Smirnov and F.S. Chuharev, FIRE6: Feynman Integral REduction with Modular Arithmetic, Comput. Phys. Commun. 247 (2020) 106877 [arXiv:1901.07808] [InSPIRE].

[50] P. Maierhöfer, J. Usovitsch and P. Uwer, Kira - A Feynman integral reduction program, Comput. Phys. Commun. 230 (2018) 99 [arXiv:1705.05610] [INSPIRE].

[51] J. Klappert, F. Lange, P. Maierhöfer and J. Usovitsch, Integral reduction with Kira 2.0 and finite field methods, Comput. Phys. Commun. 266 (2021) 108024 [arXiv:2008.06494] [INSPIRE].

[52] R.N. Lee, Presenting LiteRed: a tool for the Loop InTEgrals REDuction, arXiv:1212.2685 [INSPIRE].

[53] R.N. Lee, LiteRed 1.4: a powerful tool for reduction of multiloop integrals, J. Phys. Conf. Ser. 523 (2014) 012059 [arXiv: 1310.1145] [INSPIRE].

[54] C. Studerus, Reduze-Feynman Integral Reduction in $C++$, Comput. Phys. Commun. 181 (2010) 1293 [arXiv: 0912.2546] [INSPIRE].

[55] A. von Manteuffel and C. Studerus, Reduze 2 - Distributed Feynman Integral Reduction, arXiv: 1201.4330 [INSPIRE].

[56] S. Catani and M. Grazzini, Infrared factorization of tree level QCD amplitudes at the next-to-next-to-leading order and beyond, Nucl. Phys. B 570 (2000) 287 [hep-ph/9908523] [INSPIRE].

[57] M. Abramowitz and I. Stegun, Handbook of Mathematical Functions, With Formulas, Graphs, and Mathematical Tables, Dover Publications, Incorporated (1974).

[58] T. Huber and D. Maître, HypExp: A Mathematica package for expanding hypergeometric functions around integer-valued parameters, Comput. Phys. Commun. 175 (2006) 122 [hep-ph/0507094] [INSPIRE].

[59] E. Panzer, Algorithms for the symbolic integration of hyperlogarithms with applications to Feynman integrals, Comput. Phys. Commun. 188 (2015) 148 [arXiv:1403.3385] [INSPIRE].

[60] K. Erhardt, Modifizierte integration-by-parts Relationen im Kontext softer Gluonemission, BSc Thesis KIT, TTP, unpublished (2021). 
[61] S. Catani, D. Colferai and A. Torrini, Triple (and quadruple) soft-gluon radiation in QCD hard scattering, JHEP 01 (2020) 118 [arXiv:1908.01616] [INSPIRE].

[62] C. Anastasiou and A. Daleo, Numerical evaluation of loop integrals, JHEP 10 (2006) 031 [hep-ph/0511176] [INSPIRE].

[63] M. Czakon, Automatized analytic continuation of Mellin-Barnes integrals, Comput. Phys. Commun. 175 (2006) 559 [hep-ph/0511200] [INSPIRE].

[64] A.V. Smirnov and V.A. Smirnov, On the Resolution of Singularities of Multiple Mellin-Barnes Integrals, Eur. Phys. J. C 62 (2009) 445 [arXiv:0901.0386] [INSPIRE].

[65] X. Liu, Y.-Q. Ma and C.-Y. Wang, A Systematic and Efficient Method to Compute Multi-loop Master Integrals, Phys. Lett. B 779 (2018) 353 [arXiv:1711.09572] [INSPIRE].

[66] H.R.P. Ferguson and D.H. Bailey, A polynomial time, numerically stable integer relation algorithm, Tech. Rep. RNR-91-032 (1992). 